\title{
The Formal Bromine Atom Transfer Radical Addition of Nonactivated Bromoalkanes Using Photoredox Gold Catalysis
}

\author{
Montserrat Zidan, Terry $\mathrm{M}^{\mathrm{c} C}$ Callum, Rowan Swann and Louis Barriault* \\ Centre for Catalysis, Research and Innovation, Department of Chemistry and Biomolecular \\ Sciences, University of Ottawa, Ottawa, Ontario, Canada. \\ ${ }^{*}$ Correspondence to: Ibarriau@uottawa.ca
}

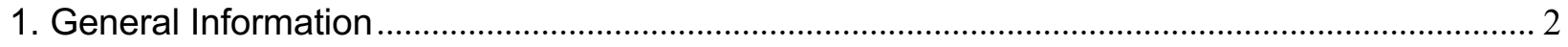

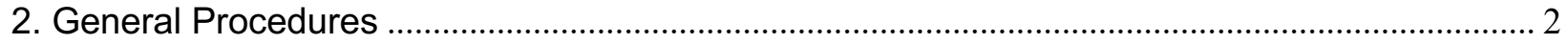

General Procedure 1. Synthesis of malonate derived bromoalkanes. ........................................... 2

General Procedure 2. Synthesis of p-toluenesulfomamide based bromoalkanes. .................... 2

General Procedure 3. Synthesis of acetal derived bromoalkanes................................................ 3

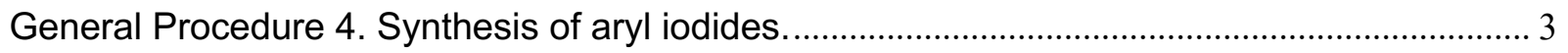

General Procedure 5. Formal Bromine Atom Transfer using Gold Photoredox Catalysis ......... 3

General Procedure 6. Formal lodine Atom Transfer using Gold Photoredox Catalysis ............ 4

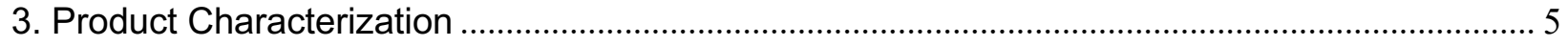

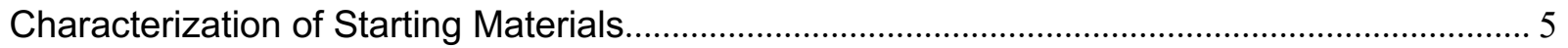

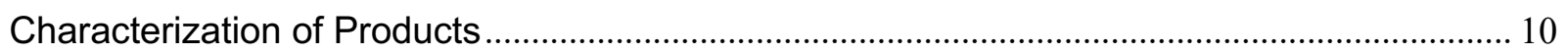

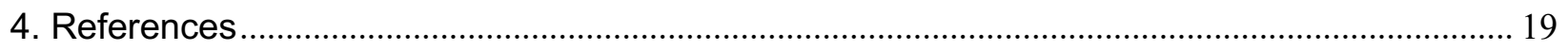

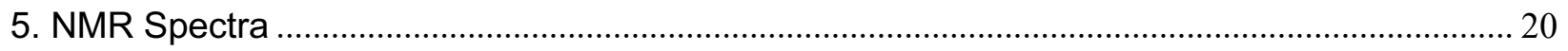




\section{General Information}

All reactions were performed under argon atmosphere in Pyrex glassware equipped with a magnetic stir bar, capped with a septum, unless otherwise indicated. All commercial reagents were used without further purification, unless otherwise noted. Reactions were monitored by thin layer chromatography (TLC) analysis. TLC plates were viewed under UV light and stained with potassium permanganate or $\mathrm{p}$-anisaldehyde staining solution. Yields refer to products isolated after purification, unless otherwise stated. Proton nuclear magnetic resonance $\left({ }^{1} \mathrm{H} N M R\right)$ spectra were recorded on a Bruker AMX $400 \mathrm{MHz}$. NMR samples were dissolved in deuterated chloroform (unless specified otherwise) and chemical shifts are reported in ppm referenced to residual undeuterated solvent. Data are reported as follows: chemical shift, multiplicity, coupling, integration. Carbon nuclear magnetic resonance $\left({ }^{13} \mathrm{C} N M R\right)$ spectra were recorded on the same Bruker instruments as in proton NMR at $101 \mathrm{MHz}$. IR spectra were recorded with an Agilent Technologies Cary 630 FTIR Spectrometer equipped with a diamond ATR module. HRMS were obtained on a Kratos Analytical Concept- Magnetic Sector Electron Impact Mass Spectrometer instrument and Micromass Q-TOF I - TOF Electrospray lonisation mass spectrometer (University of Ottawa Mass Spectrum Centre).

\section{General Procedures}

\section{General Procedure 1. Synthesis of malonate derived bromoalkanes.}

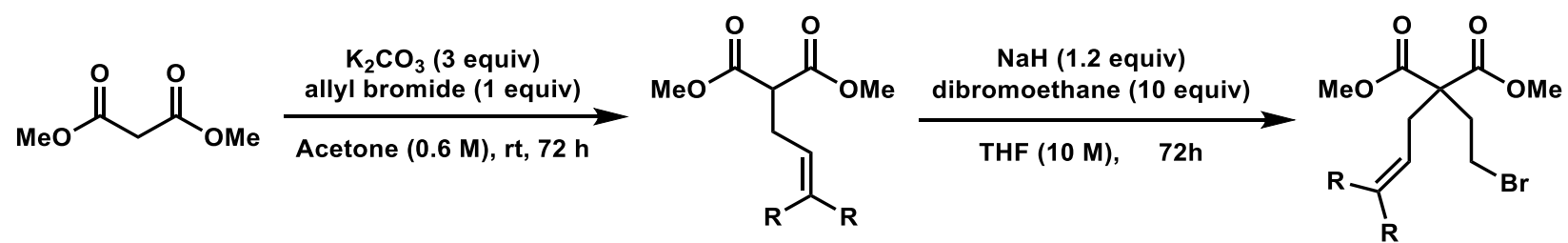

To a flame-dried round bottom flash with a magnetic stir bar, $\mathrm{K}_{2} \mathrm{CO}_{3}$ (3 equiv) was added to a solution of dimethylmalonate (1 equiv) and allyl bromide in acetone $(0.6 \mathrm{M})$. The reaction was left stirring for $72 \mathrm{~h}$ at room temperature. Once the reaction was deemed complete via TLC, it was put through a silica plug and concentrated in vacuo. Crude mixture was purified by distillation under pressure to afford the dimethyl 2-allyl malonate product. To a high-pressure tube, $\mathrm{NaH}$ (1.2 equiv) was added slowly to THF. The dimethyl 2-allyl malonate was slowly added and allowed all $\mathrm{NaH}$ to react. Once bubbling subsided, 1,2-dibromomethane was added and heated the reaction for $72 \mathrm{~h}$. Using ether and water, the organic phase was extracted and concentrated in vacuo. Crude mixture was purified by flash chromatography (0-100\% EtOAc:Hexane) where relevant fractions were combined, concentrated and characterized by proton and carbon NMR (400 and $101 \mathrm{MHz}$, respectively), HR-MS and IR.

\section{General Procedure 2. Synthesis of p-toluenesulfonamide based bromoalkanes.}

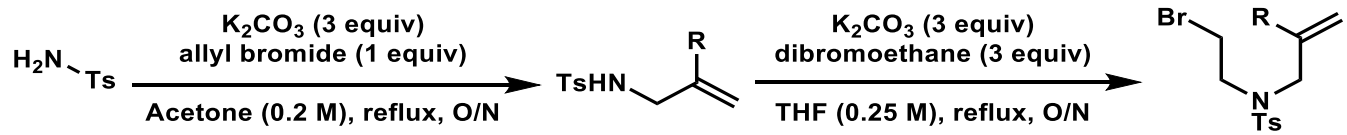

To a flame-dried round bottom flash with a magnetic stir bar, $\mathrm{K}_{2} \mathrm{CO}_{3}$ (3 equiv) was added to a solution of $p$-toluenesulfomamide (1.5 equiv) and allyl bromide (1 equiv) in acetone ( $0.2 \mathrm{M})$. The reaction was refluxed, using an oil bath as heat source, overnight. Once the reaction was deemed complete via TLC, it was put through a silica plug and concentrated in vacuo. Crude mixture was purified by distillation under pressure to afford the dimethyl $\mathrm{N}$-allyl sulfonamide product. To a high- 
pressure tube, $\mathrm{K}_{2} \mathrm{CO}_{3}$ (3 equiv) was added slowly to a solution of allyamine in $\operatorname{THF}(0.25 \mathrm{M}) .1$,2dibromomethane was added and the reaction was heated to reflux for $48 \mathrm{~h}$. Using ether and water, the organic phase was extracted and concentrated in vacuo. Crude mixture was purified by flash chromatography (0-100\% EtOAc:Hexane) where relevant fractions were combined, concentrated and characterized by proton and carbon NMR (400 and $101 \mathrm{MHz}$, respectively), HR-MS and IR.

\section{General Procedure 3. Synthesis of acetal derived bromoalkanes.}

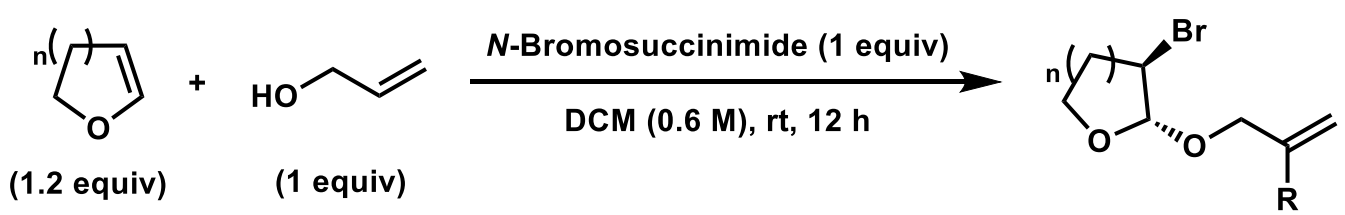

At $0^{\circ}, \mathrm{N}$-Bromosuccinimide was added to a solution of the alcohol, enol ether and DCM. Warm to room temperature and stir for $16 \mathrm{~h}$. The reaction was quench with $\mathrm{H}_{2} \mathrm{O}$ and extracted with DCM three times. Combined organic layers were washed with $\mathrm{NaHCO}_{3}$ (aq) and brine, and dried, filtered and concentrated in vacuo. Crude mixture was purified by flash chromatography $(0-100 \%$ EtOAc:Hexane) where relevant fractions were combined, concentrated and characterized by proton and carbon NMR (400 and $101 \mathrm{MHz}$, respectively), HR-MS and IR.

\section{General Procedure 4. Synthesis of aryl iodides.}

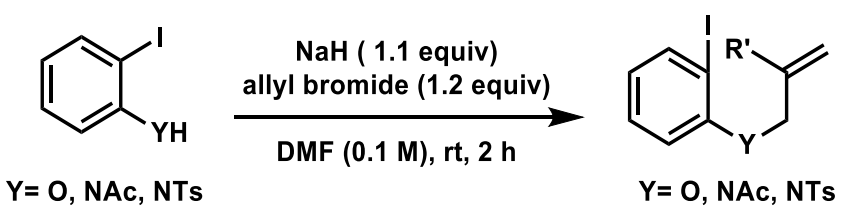

At $0^{\circ} \mathrm{C}$ add $\mathrm{NaH}$ (1.1 equiv) was added in one portion to a solution aryl iodide (1 equiv) in DMF $(0.1 \mathrm{M})$. Once the reaction stops bubbling and producing $\mathrm{H}_{2}$, add allyl bromide (1.2 equiv) and bring to room temperature. Resulting mixture was quenched with $\mathrm{H}_{2} \mathrm{O}$ after $2 \mathrm{~h}$ and extracted with EtOAc. Combined organic layers were dried, filtered, and concentrated in vacuo. Crude mixture was purified by flash chromatography (0-100\% EtOAc:Hexane) where relevant fractions were combined, concentrated and characterized by proton and carbon NMR (400 and $101 \mathrm{MHz}$, respectively), HR-MS and IR.

\section{General Procedure 5. Formal Bromine Atom Transfer using Gold Photoredox Catalysis}

To an oven dried $8 \mathrm{~mL}$ Pyrex screw-top reaction vessel was added the alkyl bromide $(0.2 \mathrm{mmol}$, 1.0 equiv), $\mathrm{Au}_{2}(\mu-\mathrm{dppm})_{2}\left(\mathrm{NTf}_{2}\right)_{2}(0.01 \mathrm{mmol}, 5 \mathrm{~mol} \%), \mathrm{MeCN}$ and $\mathrm{H}_{2} \mathrm{O}(7: 3,6 \mathrm{~mL}, 0.033 \mathrm{M})$. The reaction Pyrex vessel was capped, degassed with argon by sparging for 5 minutes, then irradiated with a UVA (365 nm) LED (LuxiGen $365 \mathrm{~nm}$ UV LED Emitter, LZ4-04UV0R, 3.8-6 W) at an approximate distance of $5 \mathrm{~mm}$ for 10 minutes. The resulting mixture was extracted with DCM and $\mathrm{H}_{2} \mathrm{O}$, and the organic layers were combined, dried, filtered and concentrated in vacuo. The crude mixture was further purified by flash chromatography (0-100\% EtOAc:Hexanes), where relevant fractions were combined, concentrated and characterized by proton and carbon NMR (400 and $101 \mathrm{MHz}$, respectively), HR-MS, and IR. 
Synthetic example: To an oven dried $8 \mathrm{~mL}$ Pyrex screw-top reaction vessel was added the alkyl bromide (1 mmol, 1.0 equiv), $\mathrm{Au}_{2}(\mu-\mathrm{dppm})_{2}\left(\mathrm{NTf}_{2}\right)_{2}(0.05 \mathrm{mmol}, 5 \mathrm{~mol} \%), \mathrm{MeCN}$ and $\mathrm{H}_{2} \mathrm{O}(7: 3$, $15 \mathrm{~mL}, 0.067 \mathrm{M})$. The reaction Pyrex vessel was capped, degassed with argon by sparging for 5 minutes, then irradiated with a UVA (365 nm) LED (LuxiGen 365 nm UV LED Emitter, LZ404UVOR, 3.8-6 W) at an approximate distance of $5 \mathrm{~mm}$ for 15 minutes. The resulting mixture was extracted with $\mathrm{DCM}$ and $\mathrm{H}_{2} \mathrm{O}$, and the organic layers were combined, dried, filtered and concentrated in vacuo. The crude mixture was further purified by flash chromatography $(0-100 \%$ EtOAc:Hexanes), where relevant fractions were combined, concentrated and characterized by proton and carbon NMR (400 and $101 \mathrm{MHz}$, respectively), HR-MS, and IR.

\section{General Procedure 6. Formal lodine Atom Transfer using Gold Photoredox Catalysis}

To an oven dried $8 \mathrm{~mL}$ Pyrex screw-top reaction vessel was added the aryl iodide $(0.2 \mathrm{mmol}, 1.0$ equiv), $\mathrm{Au}_{2}(\mu \text {-dppm })_{2}\left(\mathrm{NTf}_{2}\right)_{2}(0.01 \mathrm{mmol}, 5 \mathrm{~mol} \%), \mathrm{MeCN}$ and $\mathrm{H}_{2} \mathrm{O}(7: 3,6 \mathrm{~mL}, 0.033 \mathrm{M})$. The reaction Pyrex vessel was capped, degassed with argon by sparging for 5 minutes, then irradiated with a UVA (365 nm) LED (LuxiGen $365 \mathrm{~nm}$ UV LED Emitter, LZ4-04UV0R, 3.8-6 W) at an approximate distance of $5 \mathrm{~mm}$ for 15 minutes. The resulting mixture was extracted with DCM and $\mathrm{H}_{2} \mathrm{O}$, and the organic layers were combined, dried, filtered and concentrated in vacuo. The crude mixture was further purified by flash chromatography (0-100\% EtOAc:Hexanes), where relevant fractions were combined, concentrated and characterized by proton and carbon NMR (400 and $101 \mathrm{MHz}$, respectively), HR-MS, and IR.

Synthetic example: To an oven dried $8 \mathrm{~mL}$ Pyrex screw-top reaction vessel was added the aryl iodide (1 mmol, 1.0 equiv), $\mathrm{Au}_{2}(\mu-\mathrm{dppm})_{2}\left(\mathrm{NTf}_{2}\right)_{2}(0.05 \mathrm{mmol}, 5 \mathrm{~mol} \%), \mathrm{MeCN}$ and $\mathrm{H}_{2} \mathrm{O}(7: 3$, $15 \mathrm{~mL}, 0.067 \mathrm{M})$. The reaction Pyrex vessel was capped, degassed with argon by sparging for 5 minutes, then irradiated with a UVA (365 nm) LED (LuxiGen 365 nm UV LED Emitter, LZ404UVOR, 3.8-6 W) at an approximate distance of $5 \mathrm{~mm}$ for 20 minutes. The resulting mixture was extracted with $\mathrm{DCM}$ and $\mathrm{H}_{2} \mathrm{O}$, and the organic layers were combined, dried, filtered and concentrated in vacuo. The crude mixture was further purified by flash chromatography $(0-100 \%$ EtOAc:Hexanes), where relevant fractions were combined, concentrated and characterized by proton and carbon NMR (400 and $101 \mathrm{MHz}$, respectively), HR-MS, and IR. (xx g, xx\%) 


\section{Product Characterization}

\section{Characterization of Starting Materials}<smiles>C=CCC(CCBr)(C(=O)OC)C(=O)OC</smiles>

dimethyl-2-allyl-2-(2-bromoethyl)malonate (1a)

Synthesized according to GP1. Crude mixture was purified by flash chromatography $(0-100 \%$ EtOAc:Hexane) where relevant fractions were combined. Synthesized as a yellow oil. Contains $10 \%$ of dimethyl 2,2-diallylmalonate. $(27.9 \mathrm{~g}, 80 \%)$

IR (neat, cm ${ }^{-1}$ ): 2954 (m), 1731 (vs), 1435 (m), 1209 (s), $1164(\mathrm{~m}), 925(\mathrm{~m}) ;{ }^{1} \mathbf{H}$ NMR (400 MHz, $\left.\mathrm{CDCl}_{3}\right) \delta=5.70-5.57(\mathrm{~m}, 1 \mathrm{H}), 5.17-5.07(\mathrm{~m}, 2 \mathrm{H}), 3.74(\mathrm{~s}, 6 \mathrm{H}), 3.39-3.31(\mathrm{~m}, 2 \mathrm{H}), 2.70-$ $2.61(\mathrm{~m}, 2 \mathrm{H}), 2.49-2.39(\mathrm{~m}, 2 \mathrm{H}) \mathrm{ppm} ;{ }^{13} \mathrm{C} \mathrm{NMR}\left(101 \mathrm{MHz}, \mathrm{CDCl}_{3}\right) \delta=170.7(2 \mathrm{X} \mathrm{C}), 131.7(\mathrm{CH})$, 119.8 $\left(\mathrm{CH}_{2}\right)$, $57.6(\mathrm{C}), 52.7\left(2 \mathrm{X} \mathrm{CH}_{3}\right), 37.9\left(\mathrm{CH}_{2}\right), 36.3\left(\mathrm{CH}_{2}\right), 26.9\left(\mathrm{CH}_{2}\right)$ ppm; HRMS (ESI) m/z: $\left[\mathrm{M}^{+}+\mathrm{Na}^{+}\right]$Calc'd for $\mathrm{C}_{10} \mathrm{H}_{15} \mathrm{BrO}_{4} \mathrm{Na} 301.0046$; Found 301.0051.<smiles>C=C(C)CC(CCBr)(C(=O)OC)C(=O)OC</smiles>

dimethyl-2-(2-bromoethyl)-2-(2-methylallyl)malonate (1b)

Synthesized according to GP1. Crude mixture was purified by flash chromatography $(0-100 \%$ EtOAc:Hexane) where relevant fractions were combined. Synthesized as a yellow oil. (1.70 g, $68 \%)$

IR (neat, cm ${ }^{-1}$ ): 2956 (m), 1735 (vs), 1437 (m), 1263 (s), 1217 (s), 1203 (s), 1165 (m), 906 (m.); ${ }^{1} \mathrm{H}$ NMR $\left(400 \mathrm{MHz}_{\mathrm{CDCl}}\right) \delta=4.91(\mathrm{p}, J=1.6 \mathrm{~Hz}, 1 \mathrm{H}), 4.76(\mathrm{~m}, J=1.8,1.0 \mathrm{~Hz}, 1 \mathrm{H}), 3.75(\mathrm{~s}$, $6 \mathrm{H}), 3.38-3.28(\mathrm{~m}, 2 \mathrm{H}), 2.74(\mathrm{~d}, J=1.0 \mathrm{~Hz}, 2 \mathrm{H}), 2.50-2.43(\mathrm{~m}, 2 \mathrm{H}), 1.65(\mathrm{dd}, J=1.5,0.8 \mathrm{~Hz}$, 3H) ppm; ${ }^{13} \mathrm{C}$ NMR (101 MHz, CDCl $)_{3} \delta=171.0(2 \mathrm{X} \mathrm{C}), 139.8(\mathrm{C}), 116.3\left(\mathrm{CH}_{2}\right), 57.1(\mathrm{C}), 52.7(2$ $\left.\mathrm{X} \mathrm{CH}_{3}\right), 41.2(\mathrm{CH} 2), 36.3\left(\mathrm{CH}_{2}\right), 27.2\left(\mathrm{CH}_{2}\right), 22.9\left(\mathrm{CH}_{3}\right) \mathrm{ppm}$; HRMS (ESI) m/z: $\left[\mathrm{M}^{+}+\mathrm{Na}^{+}\right]$Calc'd for $\mathrm{C}_{11} \mathrm{H}_{17} \mathrm{BrO}_{4} \mathrm{Na} 315.0202$; Found 315.0208 .<smiles>C=CCN([As])CCBr</smiles>

$N$-allyl-N-(2-bromoethyl)-4-methylbenzenesulfonamide (1c)

Synthesized according to GP2 and characterized according to NMR comparison. ${ }^{2}$ Crude mixture was purified by flash chromatography (0-100\% EtOAc:Hexane) where relevant fractions were combined. Synthesized as a clear oil. (1.62 $\mathrm{g}, 48 \%)$

${ }^{1} \mathrm{H}$ NMR $\left(400 \mathrm{MHz}, \mathrm{CDCl}_{3}\right) \delta=7.75-7.67(\mathrm{~m}, 2 \mathrm{H}), 7.37-7.29(\mathrm{~m}, 2 \mathrm{H}), 5.68(\mathrm{ddt}, J=16.2,10.4$, $6.5 \mathrm{~Hz}, 1 \mathrm{H}), 5.22(\mathrm{dq}, J=6.6,1.3 \mathrm{~Hz}, 1 \mathrm{H}), 5.19(\mathrm{t}, J=1.3 \mathrm{~Hz}, 1 \mathrm{H}), 3.82(\mathrm{dt}, J=6.5,1.3 \mathrm{~Hz}, 2 \mathrm{H})$, $3.50-3.38(\mathrm{~m}, 4 \mathrm{H}), 2.44(\mathrm{~s}, 3 \mathrm{H}) \mathrm{ppm} ;{ }^{13} \mathrm{C}$ NMR (101 MHz, $\left.\mathrm{CDCl}_{3}\right) \delta=143.7(\mathrm{C}), 136.3(\mathrm{C}), 132.8$ $(\mathrm{CH}), 129.8(2 \times \mathrm{CH}), 127.2(2 \times \mathrm{CH}), 119.7\left(\mathrm{CH}_{2}\right), 52.0\left(\mathrm{CH}_{2}\right), 48.9\left(\mathrm{CH}_{2}\right), 29.2\left(\mathrm{CH}_{2}\right), 21.5\left(\mathrm{CH}_{3}\right)$ ppm. 
<smiles>C=C(C)CN(C)CCBr</smiles>

$\mathrm{N}$-(2-bromoethyl)-4-methyl-N-(2-methylallyl)benzenesulfonamide (1d) Synthesized according to GP2 and characterized according to NMR comparison. ${ }^{3}$ Crude mixture was purified by flash chromatography $(0-100 \%$ EtOAc:Hexane) where relevant fractions were combined. Synthesized as an amorphous solid. $(2.49 \mathrm{~g}, 89 \%)$

${ }^{1} \mathrm{H}$ NMR $\left(400 \mathrm{MHz}, \mathrm{CDCl}_{3}\right) \delta=7.73-7.67(\mathrm{~m}, 2 \mathrm{H}), 7.36-7.29(\mathrm{~m}, 2 \mathrm{H}), 4.94(\mathrm{tt}, J=1.5,0.7 \mathrm{~Hz}$, $1 \mathrm{H}), 4.90(\mathrm{p}, J=1.1 \mathrm{~Hz}, 1 \mathrm{H}), 3.70(\mathrm{~s}, 2 \mathrm{H}), 3.46-3.34(\mathrm{~m}, 4 \mathrm{H}), 2.44(\mathrm{~s}, 3 \mathrm{H}), 1.73(\mathrm{~s}, 3 \mathrm{H}) \mathrm{ppm}$; ${ }^{13} \mathrm{C}$ NMR (101 MHz, CDCl 3 ) $\delta=143.6(\mathrm{C}), 140.4(\mathrm{C}), 136.1(\mathrm{C}), 129.8(2 \mathrm{X} \mathrm{CH}), 127.1(2 \times \mathrm{CH})$, $115.3\left(\mathrm{CH}_{2}\right), 55.8\left(\mathrm{CH}_{2}\right), 49.5\left(\mathrm{CH}_{2}\right), 28.9\left(\mathrm{CH}_{2}\right), 21.5\left(\mathrm{CH}_{3}\right), 19.7(\mathrm{CH} 3) \mathrm{ppm}$.

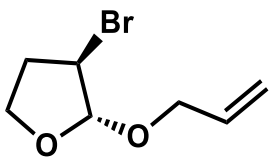

(2S,3R)-2(allyloxy)-3-bromotetrahydrofuran (1e)

Synthesized according to GP3 and characterized according to NMR comparison. ${ }^{4}$ Crude mixture was purified by flash chromatography (0-100\% EtOAc:Hexane) where relevant fractions were combined. Synthesized as a clear oil. $(1.75 \mathrm{~g}, 35 \%)$

${ }^{1} \mathrm{H}$ NMR $\left(400 \mathrm{MHz}, \mathrm{CDCl}_{3}\right) \delta=5.89$ (dddd, $\left.J=17.2,10.4,6.1,5.3 \mathrm{~Hz}, 1 \mathrm{H}\right), 5.32-5.24(\mathrm{~m}, 2 \mathrm{H})$, $5.20(\mathrm{dq}, J=10.4,1.3 \mathrm{~Hz}, 1 \mathrm{H}), 4.25(\mathrm{dd}, J=6.0,1.6 \mathrm{~Hz}, 1 \mathrm{H}), 4.22-4.14(\mathrm{~m}, 2 \mathrm{H}), 4.08(\mathrm{td}, J=$ 8.5, 3.4 Hz, 1H), 3.99 (ddt, $J=12.8,6.1,1.4 \mathrm{~Hz}, 1 \mathrm{H}), 2.73-2.61(\mathrm{~m}, 1 \mathrm{H}), 2.23$ (dddd, $J=14.0$, 7.0, 3.4, $1.7 \mathrm{~Hz}, 1 \mathrm{H}) \mathrm{ppm} ;{ }^{13} \mathrm{C}$ NMR $\left(101 \mathrm{MHz}, \mathrm{CDCl}_{3}\right) \delta=134.0(\mathrm{CH}), 117.4\left(\mathrm{CH}_{2}\right), 108.0(\mathrm{CH})$, $68.1\left(\mathrm{CH}_{2}\right), 66.7\left(\mathrm{CH}_{2}\right), 50.0(\mathrm{CH}), 33.9\left(\mathrm{CH}_{2}\right)$ ppm.<smiles>C=CCO[C@H]1OCCC[C@@H]1Br</smiles>

(2S,3R)-2(allyloxy)-3-bromotetrahydro-2H-pyr (1f)

Synthesized according to GP3 and characterized according to NMR comparison. ${ }^{5}$ Crude mixture was purified by flash chromatography (0-100\% EtOAc:Hexane) where relevant fractions were combined. Synthesized as a clear oil. (3.19 g, 64\%)

${ }^{1} \mathrm{H}$ NMR $\left(400 \mathrm{MHz}, \mathrm{CDCl}_{3}\right.$ ) $\delta=5.94$ (dddd, $J=16.9,10.4,6.1,5.2 \mathrm{~Hz}, 1 \mathrm{H}$ ), 5.33 (dq, $J=17.2$, $1.7 \mathrm{~Hz}, 1 \mathrm{H}), 5.21(\mathrm{dq}, J=10.4,1.4 \mathrm{~Hz}, 1 \mathrm{H}), 4.66(\mathrm{~d}, J=4.4 \mathrm{~Hz}, 1 \mathrm{H}), 4.27$ (ddt, $J=12.9,5.2,1.5$ $\mathrm{Hz}, 1 \mathrm{H}$ ), 4.06 (ddt, $J=12.9,6.2,1.4 \mathrm{~Hz}, 1 \mathrm{H}), 4.01$ (dt, $J=6.7,4.2 \mathrm{~Hz}, 1 \mathrm{H}), 3.93$ (ddd, $J=11.2$, 7.8, 3.2 Hz, 1H), $3.63-3.55(\mathrm{~m}, 1 \mathrm{H}), 2.46-2.36(\mathrm{~m}, 1 \mathrm{H}), 2.00-1.89(\mathrm{~m}, 2 \mathrm{H}), 1.59-1.49(\mathrm{~m}$, 1H) ppm; ${ }^{13} \mathrm{C}$ NMR (101 MHz, $\left.\mathrm{CDCl}_{3}\right) \delta=133.9(\mathrm{CH}), 117.5\left(\mathrm{CH}_{2}\right), 100.1(\mathrm{CH}), 68.7\left(\mathrm{CH}_{2}\right), 62.5$ $\left(\mathrm{CH}_{2}\right), 49.3(\mathrm{CH}), 30.0\left(\mathrm{CH}_{2}\right), 23.2\left(\mathrm{CH}_{2}\right) \mathrm{ppm}$.<smiles>C=C(C)CO[C@H]1OCCC[C@@H]1Br</smiles>

(2S,3R)-3-bromo-2-((2-methylallyl)oxy)tetrahydro-2H-pyr (1g)

Synthesized according to GP3. Crude mixture was purified by flash chromatography $(0-100 \%$ EtOAc:Hexane) where relevant fractions were combined. Synthesized as a clear oil. $(3.30 \mathrm{~g}, 70 \%)$ IR (neat, cm-1): 2948 (m), $2929(\mathrm{~m}), 2854(\mathrm{~m}), 1438$ (m), 1204 (s), 1130 (vs), 1071 (vs), 1021 (vs), 948 (s), 900 (vs), 869 (vs), 729 (vs), 711 (m); ${ }^{1} \mathrm{H}$ NMR (400 MHz, $\left.\mathrm{CDCl}_{3}\right) \delta=5.03-4.97$ (m, $1 \mathrm{H}), 4.95-4.89(\mathrm{~m}, 1 \mathrm{H}), 4.63(\mathrm{~d}, J=4.5 \mathrm{~Hz}, 1 \mathrm{H}), 4.19-4.11(\mathrm{~m}, 1 \mathrm{H}), 4.04-3.88(\mathrm{~m}, 3 \mathrm{H}), 3.58$ 
(dddd, $J=11.2,6.4,3.6,1.0 \mathrm{~Hz}, 1 \mathrm{H}$ ), $2.46-2.35(\mathrm{~m}, 1 \mathrm{H}), 1.93$ (dddd, $J=15.8,13.5,7.8,3.7 \mathrm{~Hz}$, $2 \mathrm{H}), 1.77$ (s, 3H), 1.55 (dddd, $J=13.7,11.1,6.8,3.9 \mathrm{~Hz}, 1 \mathrm{H}) \mathrm{ppm} ;{ }^{13} \mathrm{C} \mathrm{NMR}\left(101 \mathrm{MHz}, \mathrm{CDCl}_{3}\right) \delta$ = $141.4(\mathrm{C}), 112.8\left(\mathrm{CH}_{2}\right), 100.1(\mathrm{CH}), 71.6\left(\mathrm{CH}_{2}\right), 62.6\left(\mathrm{CH}_{2}\right), 49.4(\mathrm{CH}), 30.2\left(\mathrm{CH}_{2}\right), 23.4\left(\mathrm{CH}_{2}\right)$,

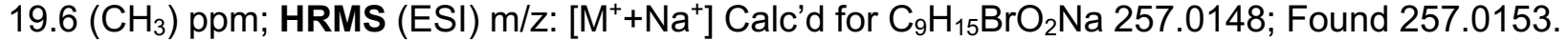

\section{1-(1-(allyloxy)-2-bromoethoxy)butane (1h)}

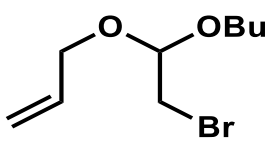

Synthesized according to GP3 and characterized according to NMR comparison. ${ }^{6}$ Crude mixture was purified by flash chromatography $(0-100 \%$ EtOAc:Hexane) where relevant fractions were combined. Synthesized as a clear oil. $(1.89 \mathrm{~g}, 67 \%)$

${ }^{1} \mathrm{H}$ NMR $\left(400 \mathrm{MHz}_{\mathrm{CDCl}}\right.$ ) $\delta=5.92$ (dddd, $\left.J=17.1,10.3,5.9,5.4 \mathrm{~Hz}, 1 \mathrm{H}\right), 5.32$ (dq, $J=17.2$, $1.6 \mathrm{~Hz}, 1 \mathrm{H}), 5.20(\mathrm{dq}, J=10.4,1.4 \mathrm{~Hz}, 1 \mathrm{H}), 4.71(\mathrm{t}, J=5.5 \mathrm{~Hz}, 1 \mathrm{H}), 4.16$ (ddt, $J=12.7,5.4,1.5$ $\mathrm{Hz}, 1 \mathrm{H}), 4.07(\mathrm{ddt}, J=12.7,5.9,1.4 \mathrm{~Hz}, 1 \mathrm{H}), 3.63(\mathrm{dt}, J=9.2,6.5 \mathrm{~Hz}, 1 \mathrm{H}), 3.51(\mathrm{dt}, J=9.2,6.6$ $\mathrm{Hz}, 1 \mathrm{H}), 3.38(\mathrm{~d}, J=5.5 \mathrm{~Hz}, 2 \mathrm{H}), 1.63-1.54(\mathrm{~m}, 3 \mathrm{H}), 1.46-1.35(\mathrm{~m}, 3 \mathrm{H}), 0.93(\mathrm{t}, J=7.4 \mathrm{~Hz}$, $4 \mathrm{H}) \mathrm{ppm} ;{ }^{13} \mathrm{C}$ NMR $\left(101 \mathrm{MHz}, \mathrm{CDCl}_{3}\right) \delta=134.1(\mathrm{CH}), 117.3\left(\mathrm{CH}_{2}\right), 101.0(\mathrm{CH}), 67.5\left(\mathrm{CH}_{2}\right), 66.6$ $\left(\mathrm{CH}_{2}\right), 31.8\left(\mathrm{CH}_{2}\right), 31.6\left(\mathrm{CH}_{2}\right), 19.3\left(\mathrm{CH}_{2}\right), 13.8\left(\mathrm{CH}_{3}\right) \mathrm{ppm}$.

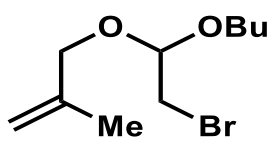

1-(2-bromo-1-((2-methylallyl)oxy)ethoxy)butane (1i)

Synthesized according to GP3. Crude mixture was purified by flash chromatography $(0-100 \%$ EtOAc:Hexane) where relevant fractions were combined. Synthesized as a clear oil. $(2.34 \mathrm{~g}, 74 \%)$ IR (neat, $\left.\mathrm{cm}^{-1}\right)$ : $2959(\mathrm{~m}), 2933(\mathrm{~m}), 2871(\mathrm{~m}), 1458(\mathrm{~m}), 1424(\mathrm{~m}), 1115(\mathrm{~s}), 1066(\mathrm{~s}), 1033$ (vs), $900(\mathrm{~s}), 683(\mathrm{~m}) ;{ }^{1} \mathrm{H}$ NMR $\left(400 \mathrm{MHz}, \mathrm{CDCl}_{3}\right) \delta=5.03-4.98(\mathrm{~m}, 1 \mathrm{H}), 4.91(\mathrm{t}, J=1.8 \mathrm{~Hz}, 1 \mathrm{H}), 4.70$ (t, $J=5.5 \mathrm{~Hz}, 1 \mathrm{H}$ ), 4.01 (dd, $J=32.7,12.4 \mathrm{~Hz}, 2 \mathrm{H}$ ), $3.62(\mathrm{dt}, J=9.1,6.6 \mathrm{~Hz}, 1 \mathrm{H}), 3.52(\mathrm{dt}, J=$ 9.2, $6.5 \mathrm{~Hz}, 1 \mathrm{H}), 3.39(\mathrm{~d}, J=5.5 \mathrm{~Hz}, 2 \mathrm{H}), 1.77(\mathrm{~s}, 3 \mathrm{H}), 1.63-1.54(\mathrm{~m}, 2 \mathrm{H}), 1.46-1.35(\mathrm{~m}, 2 \mathrm{H})$, $0.93(\mathrm{t}, J=7.4 \mathrm{~Hz}, 3 \mathrm{H}) \mathrm{ppm} ;{ }^{13} \mathrm{C}$ NMR $\left(101 \mathrm{MHz}, \mathrm{CDCl}_{3}\right) \delta=141.6(\mathrm{C}), 112.7\left(\mathrm{CH}_{2}\right), 101.0(\mathrm{CH})$, $70.5\left(\mathrm{CH}_{2}\right), 66.6\left(\mathrm{CH}_{2}\right), 31.8\left(\mathrm{CH}_{2}\right), 31.6\left(\mathrm{CH}_{2}\right), 19.6\left(\mathrm{CH}_{3}\right), 19.3\left(\mathrm{CH}_{2}\right), 13.8\left(\mathrm{CH}_{3}\right)$ ppm; HRMS (EI) $\mathrm{m} / \mathrm{z}:\left[\mathrm{M}^{+}-\mathrm{C}_{4} \mathrm{H}_{9} \mathrm{O}\right]$ Calc'd for $\mathrm{C}_{6} \mathrm{H}_{10} \mathrm{BrO}$ 176.9915; Found 176.9894.

\section{1-(allyloxy)-2-iodobenzene (1m)}

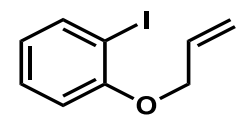

Synthesized according to GP4 and characterized according to NMR comparison. ${ }^{7}$ Crude mixture was purified by flash chromatography $(0-100 \%$ EtOAc:Hexane) where relevant fractions were combined. Synthesized as a yellow oil. (4.94 g, 97\%)

${ }^{1} \mathrm{H}$ NMR $\left(400 \mathrm{MHz}, \mathrm{CDCl}_{3}\right) \delta=7.79(\mathrm{dd}, J=7.8,1.6 \mathrm{~Hz}, 1 \mathrm{H}), 7.32-7.26(\mathrm{~m}, 1 \mathrm{H}), 6.81(\mathrm{dd}, J=$ 8.2, $1.4 \mathrm{~Hz}, 1 \mathrm{H}), 6.72(\mathrm{td}, J=7.6,1.4 \mathrm{~Hz}, 1 \mathrm{H}), 6.07$ (ddt, $J=17.2,10.6,4.8 \mathrm{~Hz}, 1 \mathrm{H}), 5.53(\mathrm{dq}, J$ $=17.2,1.7 \mathrm{~Hz}, 1 \mathrm{H}), 5.32(\mathrm{dq}, J=10.6,1.5 \mathrm{~Hz}, 1 \mathrm{H}), 4.60(\mathrm{dt}, J=4.9,1.7 \mathrm{~Hz}, 2 \mathrm{H}) \mathrm{ppm} ;{ }^{13} \mathrm{C}$ NMR $(101 \mathrm{MHz}, \mathrm{CDCl} 3) \delta=170.7(2 \times \mathrm{C}), 131.7(\mathrm{CH}), 119.8\left(\mathrm{CH}_{2}\right), 57.6(\mathrm{C}), 52.7\left(2 \times \mathrm{CH}_{3}\right), 37.9$ $\left(\mathrm{CH}_{2}\right), 36.3\left(\mathrm{CH}_{2}\right), 26.9\left(\mathrm{CH}_{2}\right) \mathrm{ppm} ;{ }^{13} \mathrm{C}$ NMR (101 MHz, $\left.\mathrm{CDCl}_{3}\right) \delta=157.1(\mathrm{C}), 139.5(\mathrm{CH}), 132.6$ $(\mathrm{CH}), 129.4(\mathrm{CH}), 122.7(\mathrm{CH}), 117.6\left(\mathrm{CH}_{2}\right), 112.5(\mathrm{CH}), 86.7(\mathrm{C}), 69.6\left(\mathrm{CH}_{2}\right) \mathrm{ppm}$. 
1-iodo-2-((2-methylallyl)oxy)benzene (1n)<smiles>C=C1COc2ccccc2C1(C)C</smiles>

Synthesized according to GP4 and characterized according to NMR comparison. ${ }^{8}$ Crude mixture was purified by flash chromatography $(0-100 \%$ EtOAc:Hexane) where relevant fractions were combined. Synthesized as a yellow oil. $(5.88 \mathrm{~g}, 96 \%)$

${ }^{1} \mathrm{H}$ NMR $\left(400 \mathrm{MHz}, \mathrm{CDCl}_{3}\right) \delta=7.80(\mathrm{dd}, J=7.8,1.6 \mathrm{~Hz}, 1 \mathrm{H}), 7.32-7.27(\mathrm{~m}, 1 \mathrm{H}), 6.82(\mathrm{dd}, J=$ 8.3, $1.4 \mathrm{~Hz}, 1 \mathrm{H}$ ), 6.72 (td, $J=7.6,1.4 \mathrm{~Hz}, 1 \mathrm{H}$ ), 5.22 (dd, $J=1.7,0.9 \mathrm{~Hz}, 1 \mathrm{H}$ ), 5.04 (hept, $J=1.5$ $\mathrm{Hz}, 1 \mathrm{H}), 4.50(\mathrm{~s}, 2 \mathrm{H}), 1.89(\mathrm{~d}, J=0.8 \mathrm{~Hz}, 3 \mathrm{H}) \mathrm{ppm} ;{ }^{13} \mathrm{C} \mathrm{NMR}\left(101 \mathrm{MHz}, \mathrm{CDCl}_{3}\right) \delta=157.1(\mathrm{C})$, $140.2(\mathrm{C}), 139.4(\mathrm{CH}), 129.3(\mathrm{CH}), 122.5(\mathrm{CH}), 112.9\left(\mathrm{CH}_{2}\right), 112.3(\mathrm{CH}), 86.5(\mathrm{C}), 72.5\left(\mathrm{CH}_{2}\right)$, $19.5\left(\mathrm{CH}_{3}\right) \mathrm{ppm}$.

\section{$\mathbf{N}$-allyl-N-(2-iodophenyl)acetamide (10)}<smiles>C=CCN(C)c1ccccc1I</smiles>

Synthesized according to GP4 and characterized according to NMR comparison. ${ }^{10}$ Crude mixture was purified by flash chromatography $(0-100 \%$ EtOAc:Hexane) where relevant fractions were combined. Synthesized as an amorphous solid. (1.40 g, 68\%)

${ }^{1} \mathrm{H}$ NMR $\left(400 \mathrm{MHz}, \mathrm{CDCl}_{3}\right) \delta=7.95(\mathrm{dd}, J=7.9,1.4 \mathrm{~Hz}, 1 \mathrm{H}), 7.39(\mathrm{td}, J=7.6,1.4 \mathrm{~Hz}, 1 \mathrm{H}), 7.18$ (dd, $J=7.8,1.6 \mathrm{~Hz}, 1 \mathrm{H}$ ), 7.08 (ddd, $J=7.9,7.4,1.6 \mathrm{~Hz}, 1 \mathrm{H}$ ), 5.90 (dddd, $J=17.1,10.1,7.8,5.5$ $\mathrm{Hz}, 1 \mathrm{H}), 5.11(\mathrm{dq}, J=10.1,1.2 \mathrm{~Hz}, 1 \mathrm{H}), 5.05(\mathrm{dq}, J=17.1,1.5 \mathrm{~Hz}, 1 \mathrm{H}), 4.85$ (ddt, $J=14.7,5.5$, $1.5 \mathrm{~Hz}, 1 \mathrm{H}), 3.59$ (ddt, $J=14.6,7.7,1.0 \mathrm{~Hz}, 1 \mathrm{H}), 1.80(\mathrm{~s}, 3 \mathrm{H}) \mathrm{ppm} ;{ }^{13} \mathbf{C} \mathbf{N M R}\left(101 \mathrm{MHz}, \mathrm{CDCl}_{3}\right)$ $\delta=169.8(\mathrm{C}), 144.7(\mathrm{C}), 140.2(\mathrm{CH}), 132.7(\mathrm{CH}), 130.5(\mathrm{CH}), 129.8(\mathrm{CH}), 129.4(\mathrm{CH}), 118.6$ $\left(\mathrm{CH}_{2}\right), 100.5(\mathrm{C}), 51.0\left(\mathrm{CH}_{2}\right), 22.8\left(\mathrm{CH}_{3}\right) \mathrm{ppm}$.

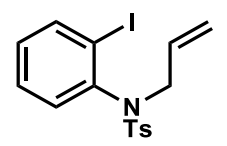

$N$-allyl-N-(2-iodophenyl)-4-methylbenzenesulfonamide (1p)

Synthesized according to GP4 and characterized according to NMR comparison. ${ }^{9}$ Crude mixture was purified by flash chromatography $(0-100 \%$ EtOAc:Hexane) where relevant fractions were combined. Synthesized as an amorphous solid. (0.8324 g, 95\%)

${ }^{1} \mathrm{H}$ NMR $\left(400 \mathrm{MHz}, \mathrm{CDCl}_{3}\right) \delta=7.91(\mathrm{dd}, J=7.9,1.5 \mathrm{~Hz}, 1 \mathrm{H}), 7.72-7.64(\mathrm{~m}, 2 \mathrm{H}), 7.32-7.29$ $(\mathrm{m}, 2 \mathrm{H}), 7.28-7.25(\mathrm{~m}, 1 \mathrm{H}), 7.02(\mathrm{ddd}, J=7.9,7.4,1.6 \mathrm{~Hz}, 1 \mathrm{H}), 6.94(\mathrm{dd}, J=7.9,1.6 \mathrm{~Hz}, 1 \mathrm{H})$, 5.87 (ddt, $J=17.0,10.1,6.8 \mathrm{~Hz}, 1 \mathrm{H}), 5.04(\mathrm{dq}, J=10.1,1.1 \mathrm{~Hz}, 1 \mathrm{H}), 4.98(\mathrm{dq}, J=17.0,1.4 \mathrm{~Hz}$, $1 \mathrm{H}), 4.24-4.07(\mathrm{~m}, 2 \mathrm{H}), 2.45(\mathrm{~s}, 3 \mathrm{H}) \mathrm{ppm} ;{ }^{13} \mathrm{C}$ NMR $\left(101 \mathrm{MHz}, \mathrm{CDCl}_{3}\right) \delta=143.7(\mathrm{C}), 141.1(\mathrm{C})$, $140.3(\mathrm{C}), 136.5(\mathrm{CH}), 132.3(\mathrm{CH}), 131.0(\mathrm{CH}), 129.9(\mathrm{CH}), 129.5(2 \mathrm{X} \mathrm{CH}), 128.6(\mathrm{CH}), 128.2(2$ $\mathrm{X} \mathrm{CH}), 119.6\left(\mathrm{CH}_{2}\right), 103.1(\mathrm{C}), 54.5\left(\mathrm{CH}_{2}\right), 21.59\left(\mathrm{CH}_{3}\right) \mathrm{ppm}$.<smiles>C=C(C)CN(C)c1ccccc1N</smiles>

$N$-(2-iodophenyl)-4-methyl-N-(2-methylallyl)benzenesulfonamide (1q)

Synthesized according to GP4. Crude mixture was purified by flash chromatography $(0-100 \%$ EtOAc:Hexane) where relevant fractions were combined. Synthesized as an amorphous solid. $(2.83 \mathrm{~g}, 78 \%)$ 
IR (neat, $\left.\mathrm{cm}^{-1}\right): 2960(\mathrm{~m}), 2926(\mathrm{~m}), 2862(\mathrm{~m}), 1599$ (s), 1476 (s), 1461 (s), 1356 (vs), 1166 (vs); ${ }^{1} \mathbf{H}$ NMR $\left(400 \mathrm{MHz}, \mathrm{CDCl}_{3}\right) \delta=7.92-7.83(\mathrm{~m}, 1 \mathrm{H}), 7.65-7.56(\mathrm{~m}, 2 \mathrm{H}), 7.31-7.26(\mathrm{~m}, 3 \mathrm{H}), 7.04-$ $6.94(\mathrm{~m}, 2 \mathrm{H}), 4.76(\mathrm{p}, J=1.5 \mathrm{~Hz}, 1 \mathrm{H}), 4.62(\mathrm{dq}, J=1.8,0.9 \mathrm{~Hz}, 1 \mathrm{H}), 4.20(\mathrm{~d}, J=14.1 \mathrm{~Hz}, 1 \mathrm{H})$, $4.04(\mathrm{~d}, J=14.1 \mathrm{~Hz}, 1 \mathrm{H}), 2.45(\mathrm{~s}, 3 \mathrm{H}), 1.91-1.81(\mathrm{~m}, 3 \mathrm{H}) \mathrm{ppm} ;{ }^{13} \mathrm{C} \mathbf{N M R}\left(101 \mathrm{MHz}, \mathrm{CDCl}_{3}\right) \delta=$ $143.7(\mathrm{C}), 141.3(\mathrm{C}), 140.6(\mathrm{CH}), 139.8(\mathrm{C}), 136.3(\mathrm{C}), 131.1(\mathrm{CH}), 129.6(\mathrm{CH}), 129.4(2 \times \mathrm{CH})$, $128.5(\mathrm{CH}), 128.2(2 \mathrm{X} \mathrm{CH}), 116.38\left(\mathrm{CH}_{2}\right), 101.7(\mathrm{C}), 57.8\left(\mathrm{CH}_{2}\right), 21.6\left(\mathrm{CH}_{3}\right), 21.0\left(\mathrm{CH}_{3}\right) \mathrm{ppm}$;

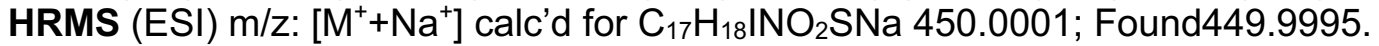<smiles>BrCCN([Te])C1C=CCCC1</smiles>

\section{N-(2-bromoethyl)-N-(cyclohex-2-en-1-yl)-4-methylbenzenesulfonamide (1r)}

Synthesized according to GP2 and characterized according to NMR comparison. ${ }^{3}$ Crude mixture was purified by flash chromatography $(0-100 \%$ EtOAc:Hexane) where relevant fractions were combined. Synthesized as an amorphous solid. $(1.25 \mathrm{~g}, 43 \%)$

${ }^{1} \mathrm{H}$ NMR $\left(400 \mathrm{MHz}, \mathrm{CDCl}_{3}\right) \delta=7.78-7.68(\mathrm{~m}, 2 \mathrm{H}), 7.36-7.28(\mathrm{~m}, 2 \mathrm{H}), 5.88-5.74(\mathrm{~m}, 1 \mathrm{H}), 4.96$ $(\mathrm{dp}, J=10.2,2.0 \mathrm{~Hz}, 1 \mathrm{H}), 4.46(\mathrm{dtd}, J=11.5,5.5,2.6 \mathrm{~Hz}, 1 \mathrm{H}), 3.70-3.62(\mathrm{~m}, 1 \mathrm{H}), 3.51-3.37$ $(\mathrm{m}, 2 \mathrm{H}), 3.32-3.19(\mathrm{~m}, 1 \mathrm{H}), 2.43(\mathrm{~s}, 3 \mathrm{H}), 2.01-1.92(\mathrm{~m}, 2 \mathrm{H}), 1.92-1.83(\mathrm{~m}, 1 \mathrm{H}), 1.82-1.72$ $(\mathrm{m}, 1 \mathrm{H}), 1.65-1.58(\mathrm{~m}, 1 \mathrm{H}), 1.42(\mathrm{tdd}, J=12.7,10.1,3.0 \mathrm{~Hz}, 1 \mathrm{H}) \mathrm{ppm} ;{ }^{13} \mathrm{C}$ NMR $(101 \mathrm{MHz}$, $\left.\mathrm{CDCl}_{3}\right) \delta=143.6(\mathrm{C}), 137.2(\mathrm{C}), 133.3(\mathrm{CH}), 129.8(2 \times \mathrm{CH}), 127.1(\mathrm{CH}), 126.8(2 \times \mathrm{CH}), 55.4$ $(\mathrm{CH}), 45.6\left(\mathrm{CH}_{2}\right), 31.1\left(\mathrm{CH}_{2}\right), 29.1\left(\mathrm{CH}_{2}\right), 24.4\left(\mathrm{CH}_{2}\right), 21.5\left(\mathrm{CH}_{3}\right), 21.5\left(\mathrm{CH}_{2}\right) \mathrm{ppm}$.<smiles>CC1=CCCCC1N(C)CCBr</smiles>

$\mathbf{N}$-(2-bromoethyl)-4-methyl-N-(2-methylcyclohex-2-en-1-yl)benzenesulfonamide (1s) Synthesized according to GP2 and characterized according to NMR comparison. ${ }^{3}$ Crude mixture was purified by flash chromatography $(0-100 \%$ EtOAc:Hexane) where relevant fractions were combined. Synthesized as an amorphous solid. (1.02 g, 46\%)

${ }^{1} \mathrm{H}$ NMR $\left(400 \mathrm{MHz}, \mathrm{CDCl}_{3}\right) \delta=7.72(\mathrm{dt}, J=8.8,2.1 \mathrm{~Hz}, 2 \mathrm{H}), 7.33-7.27(\mathrm{~m}, 2 \mathrm{H}), 5.69(\mathrm{ddq}, J=$ $5.1,3.5,1.7 \mathrm{~Hz}, 1 \mathrm{H}), 4.29-4.18(\mathrm{~m}, 1 \mathrm{H}), 3.74-3.63(\mathrm{~m}, 1 \mathrm{H}), 3.59-3.38(\mathrm{~m}, 2 \mathrm{H}), 3.26-3.15$ $(\mathrm{m}, 1 \mathrm{H}), 2.42(\mathrm{~s}, 3 \mathrm{H}), 2.06-1.85(\mathrm{~m}, 2 \mathrm{H}), 1.85-1.74(\mathrm{~m}, 1 \mathrm{H}), 1.63-1.50(\mathrm{~m}, 3 \mathrm{H}), 1.25(\mathrm{~s}, 3 \mathrm{H})$ ppm; ${ }^{13} \mathrm{C}$ NMR $\left(101 \mathrm{MHz}, \mathrm{CDCl}_{3}\right) \delta=143.4(\mathrm{C}), 137.6(\mathrm{C}), 131.7(\mathrm{C}) 129.9(\mathrm{CH}), 129.7(2 \times \mathrm{CH})$, $127.1(2 \times \mathrm{CH}), 57.7(\mathrm{CH}), 45.9\left(\mathrm{CH}_{2}\right), 30.5\left(\mathrm{CH}_{2}\right), 30.1\left(\mathrm{CH}_{2}\right), 25.0\left(\mathrm{CH}_{2}\right), 21.5\left(\mathrm{CH}_{3}\right), 20.9\left(\mathrm{CH}_{2}\right)$, $20.6\left(\mathrm{CH}_{3}\right) \mathrm{ppm}$.<smiles>COC(=O)C(CC=C(C)[N+](=O)[O-])(CCBr)C(=O)OC</smiles>

dimethyl-2-(2-bromoethyl)-2-(3-methylbut-2-en-1-yl)malonate (1t)

Synthesized according to GP1 and characterized according to NMR comparison. ${ }^{1}$ Crude mixture was purified by flash chromatography (0-100\% EtOAc:Hexane) where relevant fractions were combined. Synthesized as a yellow oil. $(1.3 \mathrm{~g}, 72 \%)$

${ }^{1} \mathrm{H}$ NMR $\left(400 \mathrm{MHz}, \mathrm{CDCl}_{3}\right) \delta=5.00-4.87(\mathrm{~m}, 1 \mathrm{H}), 3.74(\mathrm{~s}, 6 \mathrm{H}), 3.39-3.26(\mathrm{~m}, 2 \mathrm{H}), 2.63(\mathrm{dq}, J$ $=7.3,0.9 \mathrm{~Hz}, 2 \mathrm{H}), 2.49-2.36(\mathrm{~m}, 2 \mathrm{H}), 1.71(\mathrm{~d}, J=1.3 \mathrm{~Hz}, 3 \mathrm{H}), 1.63(\mathrm{~d}, J=1.2 \mathrm{~Hz}, 3 \mathrm{H}) \mathrm{ppm} ;{ }^{13} \mathrm{C}$ 
$\operatorname{NMR}\left(101 \mathrm{MHz}, \mathrm{CDCl}_{3}\right) \delta=171.0(2 \times \mathrm{C}), 136.5(\mathrm{C}), 116.9(\mathrm{CH}), 57.8(\mathrm{C}), 52.6\left(2 \mathrm{X} \mathrm{CH}_{3}\right), 36.3$ $\left(\mathrm{CH}_{2}\right), 32.1\left(\mathrm{CH}_{2}\right), 27.3\left(\mathrm{CH}_{2}\right), 26.0\left(\mathrm{CH}_{3}\right), 17.9\left(\mathrm{CH}_{3}\right) \mathrm{ppm}$.

\section{Characterization of Products}

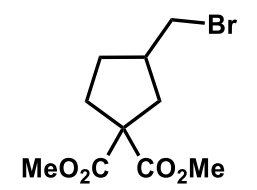

Dimethtl-3-(bromomethyl)cyclopentane-1,1-dicarboxylate (2a)

Synthesized according to GP5. Crude mixture was purified by flash chromatography (0-100\% EtOAc:Hexane) where relevant fractions were combined. Isolated as $39 \mathrm{mg}$ of a yellow oil (82 \%). IR (neat, cm $\left.{ }^{-1}\right) 2954$ (m), 1731 (vs), 1435 (m), 1259 (s), $1197(\mathrm{~m}), 1161(\mathrm{~m}) ;{ }^{1} \mathbf{H}$ NMR (400 MHz, $\left.\mathrm{CDCl}_{3}\right) \delta=3.72(\mathrm{~s}, 6 \mathrm{H}), 3.42-3.35(\mathrm{~m}, 2 \mathrm{H}), 2.56-2.41(\mathrm{~m}, 2 \mathrm{H}), 2.33(\mathrm{ddd}, J=13.4,8.5,4.8$ $\mathrm{Hz}, 1 \mathrm{H}), 2.26-2.16(\mathrm{~m}, 1 \mathrm{H}), 2.01-1.90(\mathrm{~m}, 2 \mathrm{H}), 1.54-1.41(\mathrm{~m}, 1 \mathrm{H}) \mathrm{ppm} ;{ }^{13} \mathrm{C}$ NMR $(101 \mathrm{MHz}$, $\left.\mathrm{CDCl}_{3}\right) \delta=172.5(2 \times \mathrm{C}), 60.1(\mathrm{C}), 52.8\left(2 \mathrm{X} \mathrm{CH}_{3}\right), 41.6(\mathrm{CH}), 39.3\left(\mathrm{CH}_{2}\right), 37.2\left(\mathrm{CH}_{2}\right), 33.8\left(\mathrm{CH}_{2}\right)$, $30.8\left(\mathrm{CH}_{2}\right)$ ppm; HRMS (El) m/z: [M+] calc'd for $\mathrm{C}_{10} \mathrm{H}_{15} \mathrm{BrO}_{4} 278.0154$; Found 278.0138.

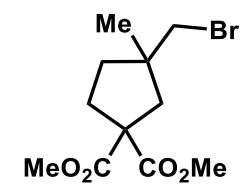

Dimethyl-3-(bromomethyl)-3-methylcyclopentane-1,1-dicarboxylate (2b)

Synthesized according to GP5. Crude mixture was purified by flash chromatography $(0-100 \%$ EtOAc:Hexane) where relevant fractions were combined. Isolated as $32 \mathrm{mg}$ of a yellow oil (55 $\%)$.

IR (neat, $\left.\mathrm{cm}^{-1}\right)$ : 2358 (s), 2342 (s), 1735 (vs), 1261 (m), 1197 (m), 1181 (m); ${ }^{1}$ H NMR (400 MHz, $\left.\mathrm{CDCl}_{3}\right) \delta=3.73(\mathrm{~s}, 7 \mathrm{H}), 3.38(\mathrm{~s}, 2 \mathrm{H}), 2.39-2.30(\mathrm{~m}, 3 \mathrm{H}), 2.17(\mathrm{~d}, J=14.4 \mathrm{~Hz}, 1 \mathrm{H}), 1.82-1.72$ $(\mathrm{m}, 1 \mathrm{H}), 1.65-1.58(\mathrm{~m}, 1 \mathrm{H}), 1.56(\mathrm{~s}, 3 \mathrm{H}), 1.17(\mathrm{~s}, 3 \mathrm{H}) \mathrm{ppm} ;{ }^{13} \mathrm{C}$ NMR $\left(101 \mathrm{MHz}, \mathrm{CDCl}_{3}\right) \delta=$ $172.8(\mathrm{C}), 172.6(\mathrm{C}), 60.3(\mathrm{C}), 52.9\left(\mathrm{CH}_{3}\right), 52.8\left(\mathrm{CH}_{3}\right), 45.1\left(\mathrm{CH}_{2}\right), 44.9\left(\mathrm{CH}_{2}\right), 44.2(\mathrm{C}), 37.5$ $\left(\mathrm{CH}_{2}\right)$, $33.6\left(\mathrm{CH}_{2}\right), 26.0\left(\mathrm{CH}_{3}\right)$ ppm; HRMS (ESI) m/z: [M+- $\left.\mathrm{CH}_{3} \mathrm{O}\right]$ calc'd for $\mathrm{C}_{10} \mathrm{H}_{13} \mathrm{BrO}_{3} 261.0126$; Found 261.0086.

\section{3-(bromomethyl)-1-tosylpyrrolidine (2c)}

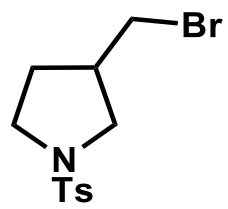

Synthesized according to GP5. Crude mixture was purified by flash chromatography $(0-100 \%$ EtOAc:Hexane) where relevant fractions were combined. Isolated as $52 \mathrm{mg}$ of white solid (82 $\%)$.

IR (neat, cm ${ }^{-1}$ ): 2973 (m), 2919 (m), 1598 (m), 1335 (vs), 1299 (s), 1235 (s). 1155 (vs), 1114 (s), 1091 (s), 1027 (s), 1013 (s), 821 (s), 811 (s), 776 (s), 707 (s), 667 (vs); ${ }^{1}$ H NMR (400 MHz, CDCl 3 ) $\delta=7.71(\mathrm{~d}, J=8.3 \mathrm{~Hz}, 2 \mathrm{H}), 7.33(\mathrm{~d}, J=8.0 \mathrm{~Hz}, 2 \mathrm{H}$ ), 3.43 (dd, $J=10.3,7.4 \mathrm{~Hz}, 1 \mathrm{H}$ ), 3.35 (ddd, $J$ = 9.9, 8.2, $4.7 \mathrm{~Hz}, 1 \mathrm{H}), 3.28-3.15(\mathrm{~m}, 3 \mathrm{H}), 3.04(\mathrm{dd}, J=10.3,6.8 \mathrm{~Hz}, 1 \mathrm{H}), 2.49$ (p, J = 7.2 Hz, $1 \mathrm{H}), 2.43(\mathrm{~s}, 3 \mathrm{H}), 2.06-1.95(\mathrm{~m}, 1 \mathrm{H}), 1.61(\mathrm{dq}, J=12.7,7.8 \mathrm{~Hz}, 1 \mathrm{H}) \mathrm{ppm} ;{ }^{13} \mathrm{C}$ NMR $(101 \mathrm{MHz}$, $\left.\mathrm{CDCl}_{3}\right) \delta=143.6(\mathrm{C}), 133.3(\mathrm{C}), 129.7(2 \mathrm{X} \mathrm{CH}), 127.6(2 \times \mathrm{CH}), 52.1\left(\mathrm{CH}_{2}\right), 47.3\left(\mathrm{CH}_{2}\right), 40.9$ 


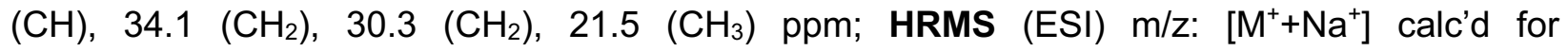
$\mathrm{C}_{12} \mathrm{H}_{16} \mathrm{BrNO}_{2} \mathrm{SNa} 339.9977$; Found 339.9983.

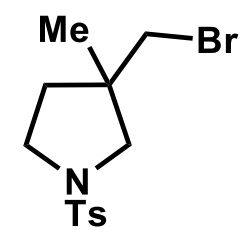

3-(bromomethyl)-3-methyl-1-tosylpyrrolidine (2d)

Synthesized according to GP5. Crude mixture was purified by flash chromatography (0-100\% EtOAc:Hexane) where relevant fractions were combined. Isolated as $33 \mathrm{mg}$ of white powder (50 $\%)$.

IR (neat, $\left.\mathrm{cm}^{-1}\right)$ : $2961(\mathrm{~m}), 2928(\mathrm{~m}), 2874(\mathrm{~m}), 1598(\mathrm{~m}), 1454(\mathrm{~m}), 1335$ (s), 1153 (vs), 1093 (s), 1052 (s), 912 (m), 816 (s), 805 (s), 731 (s), 661 (vs); ${ }^{1} \mathbf{H}$ NMR (400 MHz, $\left.\mathrm{CDCl}_{3}\right) \delta=7.71$ (d, $\mathrm{J}=$ $8.3 \mathrm{~Hz}, 2 \mathrm{H}), 7.33(\mathrm{~d}, J=7.7 \mathrm{~Hz}, 2 \mathrm{H}), 3.35-3.30(\mathrm{~m}, 2 \mathrm{H}), 3.24(\mathrm{~d}, J=10.1 \mathrm{~Hz}, 1 \mathrm{H}), 3.21(\mathrm{~s}, 2 \mathrm{H})$, $3.03(\mathrm{~d}, J=10.1 \mathrm{~Hz}, 1 \mathrm{H}), 2.43(\mathrm{~s}, 3 \mathrm{H}), 1.84(\mathrm{dt}, J=12.8,7.2 \mathrm{~Hz}, 1 \mathrm{H}), 1.70-1.60(\mathrm{~m}, 1 \mathrm{H}), 1.05$ (s, 3H) ppm; ${ }^{13} \mathrm{C}$ NMR (101 MHz, CDCl 3 ) $\delta=143.6$ (2 X C), 133.5 (2 X C), $129.7\left(2 \mathrm{X} \mathrm{CH}_{2}\right), 127.5$ (2 $\left.\mathrm{X} \mathrm{CH}_{2}\right), 57.5\left(\mathrm{CH}_{2}\right), 46.7\left(\mathrm{CH}_{2}\right), 43.4(\mathrm{C}), 41.5\left(\mathrm{CH}_{2}\right), 36.2\left(\mathrm{CH}_{2}\right), 23.2\left(\mathrm{CH}_{3}\right), 21.5\left(\mathrm{CH}_{3}\right) \mathrm{ppm}$; HRMS (ESI) m/z: [M+ $\left.{ }^{+} \mathrm{Na}^{+}\right]$calc'd for $\mathrm{C}_{13} \mathrm{H}_{18} \mathrm{BrNO}_{2} \mathrm{SNa} 354.0134$; Found 354.1102.

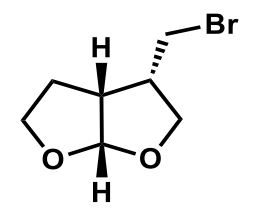

\section{3-(bromomethyl)hexahydrofuro[2,3-b]furan (2e)}

Synthesized according to GP5. Crude mixture was purified by flash chromatography $(0-100 \%$ EtOAc:Hexane) where relevant fractions were combined. Isolated as $29 \mathrm{mg}$ of clear oil $(70 \%$, $83: 17 \mathrm{dr})$ and $0.1780 \mathrm{~g}(82 \%, 83: 17 \mathrm{dr})$ when performed in a $1 \mathrm{mmol}$ scale.

IR (neat, cm $\left.{ }^{-1}\right): 2957(\mathrm{~m}), 2872(\mathrm{~m}), 2252(\mathrm{~m}), 1712(\mathrm{~m}), 1231(\mathrm{~m}), 1108(\mathrm{~m}), 1065(\mathrm{~m}), 1016(\mathrm{vs})$, 1000 (vs), 955 (m), 941 (m), 922 (s); ${ }^{1} \mathrm{H}$ NMR (400 MHz, $\left.\mathrm{CDCl}_{3}\right) \delta=5.76(\mathrm{~d}, J=4.9 \mathrm{~Hz}, 1 \mathrm{H})$, 5.73-5.71 (m, $0.05 \mathrm{H}$, minor diasteroisomer) ( $4.04(\mathrm{dd}, J=8.6,7.2 \mathrm{~Hz}, 1 \mathrm{H}), 3.90(\mathrm{dd}, J=7.6$, $6.1 \mathrm{~Hz}, 2 \mathrm{H}), 3.51(\mathrm{dd}, J=11.0,8.6 \mathrm{~Hz}, 1 \mathrm{H}), 3.38(\mathrm{dd}, J=8.0,1.7 \mathrm{~Hz}, 2 \mathrm{H}), 2.99-2.89(\mathrm{~m}, 1 \mathrm{H})$, $2.89-2.77(\mathrm{~m}, 1 \mathrm{H}), 1.97-1.90(\mathrm{~m}, 1 \mathrm{H}), 1.89-1.82(\mathrm{~m}, 1 \mathrm{H}) \mathrm{ppm} ;{ }^{13} \mathrm{C} \mathrm{NMR}\left(101 \mathrm{MHz}, \mathrm{CDCl}_{3}\right) \delta$ $=109.8(\mathrm{CH}), 71.3\left(\mathrm{CH}_{2}\right), 69.1\left(\mathrm{CH}_{2}\right), 45.6(\mathrm{CH}), 44.8(\mathrm{CH}), 29.2\left(\mathrm{CH}_{2}\right), 24.7\left(\mathrm{CH}_{2}\right)$ ppm; HRMS (EI) $\mathrm{m} / \mathrm{z}$ : $\left[\mathrm{M}^{+}-\mathrm{H}^{+}\right]$calc'd for $\mathrm{C}_{7} \mathrm{H}_{10} \mathrm{BrO}_{2} 204.9864$; Found 204.9832.

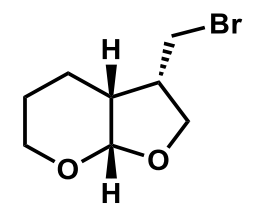

\section{3-(bromomethyl)hexahydrofuro[2,3-b]pyran (2f)}

Synthesized according to GP5. Crude mixture was purified by flash chromatography (0-100\% EtOAc:Hexane) where relevant fractions were combined. Isolated as $24 \mathrm{mg}$ of clear oil (54\%, 89:11 dr).

IR (neat, cm $\left.{ }^{-1}\right): 2937(\mathrm{~m}), 2872(\mathrm{~m}), 2360(\mathrm{~s}), 2341(\mathrm{~m}), 1148(\mathrm{~m}), 1048(\mathrm{~m}), 1024$ (vs); ${ }^{1} \mathbf{H}$ NMR $\left(400 \mathrm{MHz}, \mathrm{CDCl}_{3}\right) \delta=5.26(\mathrm{~d}, J=3.7 \mathrm{~Hz}, 1 \mathrm{H}), 5.05(\mathrm{~d}, J=3.7 \mathrm{~Hz}, 0.09 \mathrm{H}$, minor diastereoisomer) $4.04(\mathrm{t}, J=8.3 \mathrm{~Hz}, 1 \mathrm{H}), 3.84-3.72(\mathrm{~m}, 2 \mathrm{H}), 3.62(\mathrm{dtd}, J=11.3,3.9,1.5 \mathrm{~Hz}$, $1 \mathrm{H}), 3.48-3.36(\mathrm{~m}, 2 \mathrm{H}), 2.83(\mathrm{pd}, J=8.2,6.4 \mathrm{~Hz}, 1 \mathrm{H}), 2.15(\mathrm{dtd}, J=9.8,6.4,3.6 \mathrm{~Hz}, 1 \mathrm{H}), 1.84$ $-1.73(\mathrm{~m}, 1 \mathrm{H}), 1.62-1.55(\mathrm{~m}, 2 \mathrm{H}), 1.54-1.46(\mathrm{~m}, 1 \mathrm{H}) \mathrm{ppm} ;{ }^{13} \mathrm{C}$ NMR $\left(101 \mathrm{MHz}^{\mathrm{CDCl}} \mathrm{CD}_{3}\right) \delta=$ 
101.7 (CH), $69.3\left(\mathrm{CH}_{2}\right), 61.4\left(\mathrm{CH}_{2}\right), 43.8(\mathrm{CH}), 37.6(\mathrm{CH}), 30.7\left(\mathrm{CH}_{2}\right), 22.8\left(\mathrm{CH}_{2}\right), 19.0\left(\mathrm{CH}_{2}\right) \mathrm{ppm}$; HRMS (El) m/z: [M+-HBr] calc'd for $\mathrm{C}_{8} \mathrm{H}_{12} \mathrm{O}_{2}$ 140.0871; Found 140.0837.

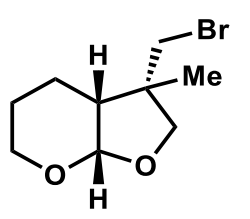

3-(bromomethyl)-3-methylhexahydro-4H-furo[2,3-b]pyran (2g)

Synthesized according to GP5. Crude mixture was purified by flash chromatography $(0-100 \%$ EtOAc:Hexane) where relevant fractions were combine. Isolated as $16 \mathrm{mg}$ of clear oil $(34 \%, 93: 7$ dr).

IR (neat, $\mathrm{cm}^{-1}$ ): $2932(\mathrm{~m}), 2870(\mathrm{~m}), 1383(\mathrm{~m}), 1241(\mathrm{~m}), 1142$ (s), $1089(\mathrm{~s}), 1044$ (s), 1024 (vs), 971 (s), 929 (s), 891 (s); ${ }^{1} \mathrm{H}$ NMR $\left(400 \mathrm{MHz}, \mathrm{CDCl}_{3}\right) \delta=5.37(\mathrm{~d}, J=3.8 \mathrm{~Hz}, 1 \mathrm{H}), 4.01$ (d, $J=8.5$ $\mathrm{Hz}, 1 \mathrm{H}), 3.81-3.73(\mathrm{~m}, 1 \mathrm{H}), 3.61-3.54(\mathrm{~m}, 3 \mathrm{H}), 3.42(\mathrm{~d}, \mathrm{~J}=10.0 \mathrm{~Hz}, 1 \mathrm{H}), 1.96-1.82(\mathrm{~m}, 2 \mathrm{H})$, $1.62-1.51(\mathrm{~m}, 3 \mathrm{H}), 1.29(\mathrm{~s}, 3 \mathrm{H}) \mathrm{ppm} ;{ }^{13} \mathrm{C}$ NMR $\left(101 \mathrm{MHz}, \mathrm{CDCl}_{3}\right) \delta=101.8(\mathrm{CH}), 75.6\left(\mathrm{CH}_{2}\right)$, $61.6\left(\mathrm{CH}_{2}\right), 45.3(\mathrm{C}), 44.11(\mathrm{CH}), 39.6\left(\mathrm{CH}_{2}\right), 24.2\left(\mathrm{CH}_{3}\right), 22.8\left(\mathrm{CH}_{2}\right), 20.3\left(\mathrm{CH}_{2}\right)$ ppm; HRMS (ESI) $\mathrm{m} / \mathrm{z}:\left[\mathrm{M}^{+}+\mathrm{Na}^{+}\right]$calc'd for $\mathrm{C}_{9} \mathrm{H}_{15} \mathrm{BrO}_{2} \mathrm{Na} 257.0148$; Found 257.0153.<smiles>CCCO[C@H]1C[C@@H](CBr)CO1</smiles>

\section{3-(bromomethyl)-2-butoxytetrahydrofuran (2h)}

Synthesized according to GP5. Crude mixture was purified by flash chromatography (0-100\% EtOAc:Hexane) where relevant fractions were combined. Isolated as $20 \mathrm{mg}$ of a clear oil (42\%, 5:1 dr).

IR (neat, $\mathrm{cm}^{-1}$ ): 2956 (s), 2925 (vs), 2885 (s), 1464 (m); ${ }^{1} \mathrm{H}$ NMR (400 MHz, CDCl $) \delta=5.14$ (dd, $J=5.3,1.6 \mathrm{~Hz}, 1 \mathrm{H}), 4.05$ (ddd, $J=10.7,8.8,7.4 \mathrm{~Hz}, 1 \mathrm{H}), 3.75-3.61(\mathrm{~m}, 2 \mathrm{H}), 3.56-3.43(\mathrm{~m}$, $2 \mathrm{H}), 3.40-3.31(\mathrm{~m}, 2 \mathrm{H}), 2.90-2.83(\mathrm{~m}, 0.2 \mathrm{H}$, minor diastereoisomer $2.75-2.63(\mathrm{~m}, 1 \mathrm{H}$, major diastereoisomer), 2.21 (ddd, $J=13.5,9.7,5.3 \mathrm{~Hz}, 1 \mathrm{H}$, major diastereoisomer), 2.09 (ddd, $J=13.5,9.7,5.3 \mathrm{~Hz}, 0.2 \mathrm{H}$, minor diastereoisomer) 1.78 (ddd, $J=13.6,4.5,1.5 \mathrm{~Hz}, 0.2 \mathrm{H}$, minor diastereoisomer) 1.72 (ddd, $J=13.6,4.5,1.5 \mathrm{~Hz}, 1 \mathrm{H}$, major diastereoisomer), 1.59 $1.49(\mathrm{~m}, 3 \mathrm{H}), 1.35$ (dtd, $J=15.4,7.7,5.2 \mathrm{~Hz}, 3 \mathrm{H}), 0.91(\mathrm{td}, J=7.4,1.9 \mathrm{~Hz}, 4 \mathrm{H}) \mathrm{ppm}$;

Major diastereoisomer: ${ }^{13} \mathrm{C}$ NMR $\left(101 \mathrm{MHz}, \mathrm{CDCl}_{3}\right) \delta=104.0(\mathrm{CH}), 71.2\left(\mathrm{CH}_{2}\right), 67.2\left(\mathrm{CH}_{2}\right)$, $40.8(\mathrm{CH}), 38.4\left(\mathrm{CH}_{2}\right), 35.8\left(\mathrm{CH}_{2}\right), 31.8\left(\mathrm{CH}_{2}\right), 19.4\left(\mathrm{CH}_{2}\right), 13.8\left(\mathrm{CH}_{3}\right) \mathrm{ppm}$;

Minor diastereoisomer: ${ }^{13} \mathrm{C}$ NMR $\left(101 \mathrm{MHz}, \mathrm{CDCl}_{3}\right) \delta=104.0(\mathrm{CH}), 70.4\left(\mathrm{CH}_{2}\right), 67.1\left(\mathrm{CH}_{2}\right), 40.0$ $(\mathrm{CH}), 38.5\left(\mathrm{CH}_{2}\right), 36.0\left(\mathrm{CH}_{2}\right), 31.8\left(\mathrm{CH}_{2}\right), 19.4\left(\mathrm{CH}_{2}\right), 13.8\left(\mathrm{CH}_{3}\right) \mathrm{ppm}$;

HRMS (ESI) m/z: [M+Na] calc'd for $\mathrm{C}_{9} \mathrm{H}_{17} \mathrm{BrO}_{2} \mathrm{Na} 259.0304$; Found 259.0310.

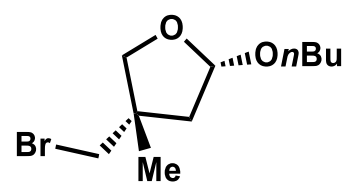

\section{3-(bromomethyl)-2-butoxy3-methyltetrahydrofuran (2i)}

Synthesized according to GP5. Crude mixture was purified by flash chromatography $(0-100 \%$ EtOAc:Hexane) where relevant fractions were combined. Isolated as $12 \mathrm{mg}$ of a clear oil $(23 \%$, 90:10 dr). 
IR (neat, $\left.\mathrm{cm}^{-1}\right): 2958(\mathrm{~m}), 2931(\mathrm{~m}), 2869(\mathrm{~m}), 1458(\mathrm{~m}), 1443(\mathrm{~m}), 1380(\mathrm{~m}), 1250(\mathrm{~m}), 1098(\mathrm{~s})$, 1084 (s), 1030 (vs), 1016 (vs), 923 (s); ${ }^{1} \mathrm{H}$ NMR (400 MHz, CDCl $)$ $\delta=5.17$ (dd, J = 5.3, $2.3 \mathrm{~Hz}$, $1 \mathrm{H}$, major diastereoisomer), $5.14(\mathrm{dd}, J=5.3,2.3 \mathrm{~Hz}, 0.2 \mathrm{H}$, minor diastereoisomer) $3.85(\mathrm{~d}$, $J=8.7 \mathrm{~Hz}, 1 \mathrm{H}$, major diastereoisomer), $3.80(\mathrm{~d}, J=8.7 \mathrm{~Hz}, 0.2 \mathrm{H}$, minor diastereoisomer) ( $3.69-3.63(\mathrm{~m}, 1 \mathrm{H}), 3.61-3.57(\mathrm{~m}, 2 \mathrm{H}), 3.52(\mathrm{~d}, J=9.8 \mathrm{~Hz}, 1 \mathrm{H}), 3.39-3.32(\mathrm{~m}, 1 \mathrm{H}), 1.98(\mathrm{~d}, J$ $=5.3 \mathrm{~Hz}, 0.2 \mathrm{H}$, minor diastereoisomer), $1.95(\mathrm{~d}, J=5.3 \mathrm{~Hz}, 1 \mathrm{H}$, major diastereoisomer), 1.92 (d, $J=2.3 \mathrm{~Hz}, 1 \mathrm{H}$, major diastereoisomer), $1.88(\mathrm{~d}, J=2.3 \mathrm{~Hz}, 0.2 \mathrm{H}$, minor diastereoisomer), $1.55-1.51(\mathrm{~m}, 2 \mathrm{H}), 1.41-1.31(\mathrm{~m}, 3 \mathrm{H}), 1.29(\mathrm{~s}, 0.6 \mathrm{H}$, minor diastereoisomer), $1.25(\mathrm{~s}, 3 \mathrm{H}$, major diastereoisomer), $0.92(\mathrm{t}, \mathrm{J}=7.4 \mathrm{~Hz}, 4 \mathrm{H}) \mathrm{ppm} ;{ }^{13} \mathbf{C}$ NMR $\left(101 \mathrm{MHz}, \mathrm{CDCl}_{3}\right) \delta=104.6$ $\left(\mathrm{CH}\right.$, major diastereoisomer) $104.5\left(\mathrm{CH}\right.$, minor diastereoisomer), $76.4\left(\mathrm{CH}_{2}\right), 67.4\left(\mathrm{CH}_{2}\right), 45.2$ $\left(\mathrm{CH}_{2}\right), 43.5(\mathrm{C}) 43.0\left(\mathrm{CH}_{2}\right), 31.8\left(\mathrm{CH}_{2}\right), 24.8\left(\mathrm{CH}_{3}\right), 19.4\left(\mathrm{CH}_{2}\right), 13.8\left(\mathrm{CH}_{3}\right) \mathrm{ppm} ; \mathrm{HRMS}(\mathrm{ESI}) \mathrm{m} / \mathrm{z}$ : $\left[\mathrm{M}^{+}-\mathrm{C}_{4} \mathrm{H}_{9} \mathrm{O}\right]$ calc'd for $\mathrm{C}_{6} \mathrm{H}_{10} \mathrm{BrO}$ 176.9915; Found 176.9910.<smiles>ICC1COc2ccccc21</smiles>

3-(iodomethyl)-2,3-dihydrobenzofuran (2m)

Synthesized according to GP6. Crude mixture was purified by flash chromatography (0-100\% EtOAc:Hexane) where relevant fractions were combined. Isolated as $32 \mathrm{mg}$ of a yellow oil $(62 \%)$ \& $0.1367 \mathrm{~g} \mathrm{(53 \% )} \mathrm{when} \mathrm{performed} \mathrm{in} \mathrm{a} 1 \mathrm{mmol}$ scale.

IR (neat, cm-1): $2965(\mathrm{~m}), 2887(\mathrm{~m}), 1596(\mathrm{~m}), 1479(\mathrm{~s}), 1461(\mathrm{~s}), 1229(\mathrm{~s}), 1161(\mathrm{~m}), 1100(\mathrm{~m})$, 965 (s), 842 (m), 746 (vs); ${ }^{1} \mathrm{H}$ NMR (400 MHz, $\left.\mathrm{CDCl}_{3}\right) \delta=7.21$ (td, J = 7.6, $5.8 \mathrm{~Hz}, 2 \mathrm{H}$ ), $6.93-$ $6.86(\mathrm{~m}, 1 \mathrm{H}), 6.81(\mathrm{~d}, J=7.9 \mathrm{~Hz}, 1 \mathrm{H}), 4.65(\mathrm{t}, J=9.1 \mathrm{~Hz}, 1 \mathrm{H}), 4.34(\mathrm{dd}, J=9.4,5.5 \mathrm{~Hz}, 1 \mathrm{H}), 3.85$ (tt, $J=9.5,4.9 \mathrm{~Hz}, 1 \mathrm{H}), 3.46(\mathrm{dd}, J=9.9,4.3 \mathrm{~Hz}, 1 \mathrm{H}), 3.22(\mathrm{t}, J=9.9 \mathrm{~Hz}, 1 \mathrm{H}) \mathrm{ppm} ;{ }^{13} \mathrm{C}$ NMR $(101$

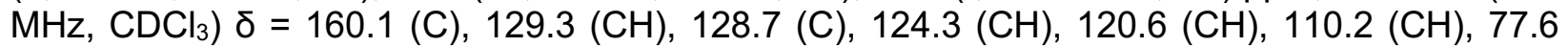
$\left(\mathrm{CH}_{2}\right), 44.8(\mathrm{CH}), 9.0\left(\mathrm{CH}_{2}\right)$ ppm; HRMS (El) m/z: [M+] calc'd for $\mathrm{C}_{9} \mathrm{H}_{9} \mathrm{IO}$ 259.9698; Found 259.9713.

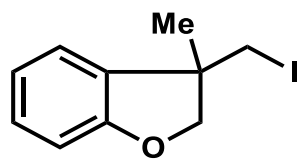

\section{3-(iodomethyl)-3-methyl-2,3-dihydrobenzofuran (2n)}

Synthesized according to GP6. Crude mixture was purified by flash chromatography $(0-100 \%$ EtOAc:Hexane) where relevant fractions were combined. Isolated as $25 \mathrm{mg}$ of a yellow oil (45\%). IR (neat, $\left.\mathrm{cm}^{-1}\right): 2962$ (m), 2924 (m), 2879 (m), 1596 (s), 1478 (vs), 1459 (s), 1209 (vs), 1016 (s), 975 (vs), 838 (s), 747 (vs); ${ }^{1} \mathrm{H}$ NMR $\left(400 \mathrm{MHz}, \mathrm{CDCl}_{3}\right) \delta=7.19$ (ddd, $J=8.0,7.4,1.4 \mathrm{~Hz}, 1 \mathrm{H}$ ), 7.12 (ddd, $J=7.4,1.4,0.6 \mathrm{~Hz}, 1 \mathrm{H}), 6.91$ (td, $J=7.4,1.0 \mathrm{~Hz}, 1 \mathrm{H}), 6.81$ (dt, $J=8.0,0.8 \mathrm{~Hz}, 1 \mathrm{H}$ ), $4.49(\mathrm{~d}, J=9.1 \mathrm{~Hz}, 1 \mathrm{H}), 4.17(\mathrm{dt}, J=9.1,0.6 \mathrm{~Hz}, 1 \mathrm{H}), 3.39(\mathrm{~s}, 2 \mathrm{H}), 1.52(\mathrm{~s}, 3 \mathrm{H}) \mathrm{ppm} ;{ }^{13} \mathrm{C}$ NMR $\left(101 \mathrm{MHz}, \mathrm{CDCl}_{3}\right) \delta={ }^{13} \mathrm{C}$ NMR $(101 \mathrm{MHz}$, Chloroform-d) $\delta=159.5(\mathrm{C}), 131.6(\mathrm{C}), 129.2(\mathrm{CH})$, 122.7 (CH), $120.7(\mathrm{CH}), 110.3(\mathrm{CH}), 82.9\left(\mathrm{CH}_{2}\right), 45.8(\mathrm{C}), 25.3\left(\mathrm{CH}_{3}\right), 18.2\left(\mathrm{CH}_{2}\right)$ ppm; HRMS (EI) $\mathrm{m} / \mathrm{z}$ : $\left[\mathrm{M}^{+}\right]$calc'd for $\mathrm{C}_{10} \mathrm{H}_{11} \mathrm{IO} 274.9855$; Found 274.9889.<smiles>CN1CC(CI)c2ccccc21</smiles>

1-(3-(iodomethyl)indolin-1-yl)ethan-1-one (2o)

Synthesized according to GP6. Crude mixture was purified by flash chromatography $(0-100 \%$ EtOAc:Hexane) where relevant fractions were combined. Isolated as $33 \mathrm{mg}$ of a yellow oil (54\%). IR (neat, cm-1): 2959 (m), 2925 (m), 1662 (vs), 1596 (s), 1481 (vs), 1462 (m), 1402 (vs), 1344 (m), $1287(\mathrm{~m}), 755(\mathrm{~s}) ;{ }^{1} \mathrm{H}$ NMR $\left(400 \mathrm{MHz}, \mathrm{CDCl}_{3}\right) \delta=8.20(\mathrm{~d}, J=8.1 \mathrm{~Hz}, 1 \mathrm{H}), 7.31-7.27(\mathrm{~m}$, 
$1 \mathrm{H}), 7.19(\mathrm{~d}, J=7.4 \mathrm{~Hz}, 1 \mathrm{H}), 7.05(\mathrm{td}, J=7.5,1.1 \mathrm{~Hz}, 1 \mathrm{H}), 4.21(\mathrm{dd}, J=10.7,9.2 \mathrm{~Hz}, 1 \mathrm{H}), 3.84$ (dd, $J=10.7,5.2 \mathrm{~Hz}, 1 \mathrm{H}), 3.76(\mathrm{tt}, J=9.2,4.3 \mathrm{~Hz}, 1 \mathrm{H}), 3.52(\mathrm{dd}, J=10.1,3.7 \mathrm{~Hz}, 1 \mathrm{H}), 3.19(\mathrm{t}, J$ $=9.9 \mathrm{~Hz}, 1 \mathrm{H}), 2.26(\mathrm{~s}, 3 \mathrm{H}) \mathrm{ppm} ;{ }^{13} \mathrm{C}$ NMR $\left(101 \mathrm{MHz}, \mathrm{CDCl}_{3}\right) \delta=168.6(\mathrm{C}), 142.9(\mathrm{C}), 132.3(\mathrm{C})$, $129.0(\mathrm{CH}), 123.8(\mathrm{CH}), 123.8(\mathrm{CH}), 117.4(\mathrm{CH}), 56.2\left(\mathrm{CH}_{2}\right), 42.8(\mathrm{CH}), 24.3\left(\mathrm{CH}_{3}\right), 9.9(\mathrm{CH})$ ppm; HRMS (El) m/z: [M+] calc'd for $\mathrm{C}_{11} \mathrm{H}_{12} \mathrm{INO} 300.9964$; Found 300.9948.

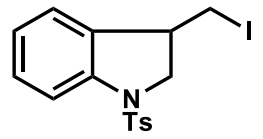

\section{3-(iodomethyl)-1-tosylindoline (2p)}

Synthesized according to GP6. Crude mixture was purified by flash chromatography $(0-100 \%$ EtOAc:Hexane) where relevant fractions were combined. Isolated as $51 \mathrm{mg}$ of white solid (62\%). IR (neat, cm-1): 2953 (m), 2923 (s), $2854(\mathrm{~m}), 1599(\mathrm{~m}), 1477(\mathrm{~m}), 1461(\mathrm{~m}), 1355$ (s), 1167 (vs), $1108(\mathrm{~m}), 1092(\mathrm{~m}), 756(\mathrm{~m}), 664(\mathrm{~s}) ;{ }^{1} \mathbf{H}$ NMR $\left(400 \mathrm{MHz}, \mathrm{CDCl}_{3}\right) \delta=7.72-7.61(\mathrm{~m}, 3 \mathrm{H}), 7.31-$ $7.19(\mathrm{~m}, 3 \mathrm{H}), 7.14-6.97(\mathrm{~m}, 2 \mathrm{H}), 4.11-3.99(\mathrm{~m}, 1 \mathrm{H}), 3.79(\mathrm{ddd}, J=11.4,5.0,1.6 \mathrm{~Hz}, 1 \mathrm{H}), 3.53$ - $3.37(\mathrm{~m}, 1 \mathrm{H}), 3.25-3.16(\mathrm{~m}, 1 \mathrm{H}), 2.65(\mathrm{td}, J=10.1,1.5 \mathrm{~Hz}, 1 \mathrm{H}), 2.37(\mathrm{~s}, 3 \mathrm{H}) \mathrm{ppm} ;{ }^{13} \mathrm{C}$ NMR (101 MHz, CDCl 3 ) $\delta{ }^{13} \mathrm{C}$ NMR (101 MHz, Chloroform-d) $\delta 144.3$ (C), 142.0 (C), 133.8 (C), 132.9 $(\mathrm{C}), 129.8(2 \times \mathrm{CH}), 129.1(\mathrm{CH}), 127.3(2 \times \mathrm{CH}), 124.5(\mathrm{CH}), 123.9(\mathrm{CH}), 115.5(\mathrm{CH}), 56.5\left(\mathrm{CH}_{2}\right)$, $43.0(\mathrm{CH}), 21.5\left(\mathrm{CH}_{3}\right), 8.7\left(\mathrm{CH}_{2}\right)$ ppm; HRMS (EI) m/z: [M+] calc'd for $\mathrm{C}_{16} \mathrm{H}_{16} \mathrm{INO}_{2} \mathrm{~S} 412.9946$; Found 412.9923.

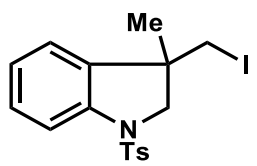

3-(iodomethyl)-3-methyl-1-tosylindoline (2q)

Synthesized according to GP6. Crude mixture was purified by flash chromatography $(0-100 \%$ EtOAc:Hexane) where relevant fractions were combined. Isolated as $38 \mathrm{mg}$ of white solid oil (45\%).

IR (neat, cm-1): 2955 (m), 2920 (s), $2854(\mathrm{~m}), 1601(\mathrm{~m}), 1480(\mathrm{~m}), 1455(\mathrm{~m}), 1361(\mathrm{~s}), 1176$ (vs), $1111(\mathrm{~m}), 1097(\mathrm{~m}), 758(\mathrm{~m}), 669$ (s); ${ }^{1} \mathbf{H}$ NMR (400 MHz, CDCl$) ~ \delta=7.75-7.64(\mathrm{~m}, 3 \mathrm{H}), 7.29-$ $7.24(\mathrm{~m}, 3 \mathrm{H}), 7.04-7.00(\mathrm{~m}, 2 \mathrm{H}), 3.97(\mathrm{~d}, J=11.1 \mathrm{~Hz}, 1 \mathrm{H}), 3.57(\mathrm{dd}, J=11.1,1.1 \mathrm{~Hz}, 1 \mathrm{H}), 3.14$ (dd, $J=10.1,1.1 \mathrm{~Hz}, 1 \mathrm{H}), 2.82(\mathrm{~d}, J=10.1 \mathrm{~Hz}, 1 \mathrm{H}), 2.37(\mathrm{~s}, 3 \mathrm{H}), 1.32(\mathrm{~s}, 3 \mathrm{H}) \mathrm{ppm} ;{ }^{13} \mathrm{C}$ NMR $(101$ $\mathrm{MHz}_{\mathrm{CDCl}}$ ) $\delta=144.3(\mathrm{C}), 141.2(\mathrm{C}), 135.4(\mathrm{C}), 134.0(\mathrm{C}), 129.8(2 \times \mathrm{CH}), 129.1(\mathrm{CH}) 127.3(2$ $\mathrm{X} \mathrm{CH}) 123.9(\mathrm{CH}), 122.9(\mathrm{CH}), 115.2(\mathrm{CH}), 62.2\left(\mathrm{CH}_{2}\right), 44.2(\mathrm{C}), 26.0\left(\mathrm{CH}_{3}\right), 21.6\left(\mathrm{CH}_{3}\right), 18.2$ $\left(\mathrm{CH}_{2}\right)$ ppm; HRMS (ESI) m/z: [M+N $\left.{ }^{+} \mathrm{Na}^{+}\right]$Calc'd for $\mathrm{C}_{17} \mathrm{H}_{18} \mathrm{INO}_{2} \mathrm{SNa} 450.000$; Found 449.9995.

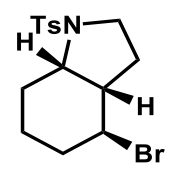

(3aS,7aS)-4-bromo-1-tosyloctahydro-1H-indole (2r)

Synthesized according to GP5. Crude mixture was purified by flash chromatography (0-100\% EtOAc:Hexane) where relevant fractions were combined. Isolated as $21 \mathrm{mg}$ of a yellow oil as a $1: 1$ mixture of diastereoisomers (29\%). Characterized according to NMR comparaison. ${ }^{3}$

${ }^{1} \mathrm{H}$ NMR $\left(400 \mathrm{MHz}, \mathrm{CDCl}_{3}\right) \delta=7.73-7.67(\mathrm{~m}, 2 \mathrm{H}), 7.35-7.28(\mathrm{~m}, 2 \mathrm{H}), 4.18(\mathrm{dt}, \mathrm{J}=12.5,5.1$ $\mathrm{Hz}, 1 \mathrm{H}), 3.72(\mathrm{dt}, J=11.1,6.3 \mathrm{~Hz}, 1 \mathrm{H}), 3.61-3.54(\mathrm{~m}, 1 \mathrm{H}), 3.14(\mathrm{td}, J=9.8,8.4 \mathrm{~Hz}, 1 \mathrm{H}), 2.44$ $(\mathrm{s}, 3 \mathrm{H}), 2.20-2.08(\mathrm{~m}, 1 \mathrm{H}), 2.08-1.96(\mathrm{~m}, 4 \mathrm{H}), 1.83-1.69(\mathrm{~m}, 2 \mathrm{H}), 1.43-1.20(\mathrm{~m}, 3 \mathrm{H}) \mathrm{ppm}$; ${ }^{13} \mathrm{C}$ NMR $\left(101 \mathrm{MHz}, \mathrm{CDCl}_{3}\right) \delta=143.4(\mathrm{C}), 135.3(\mathrm{C}), 129.8(2 \mathrm{X} \mathrm{CH}), 127.2(2 \mathrm{X} \mathrm{CH}), 59.8(\mathrm{CH})$, 
$50.0(\mathrm{CH}), 46.0\left(\mathrm{CH}_{2}\right), 45.4(\mathrm{CH}), 31.8\left(\mathrm{CH}_{2}\right), 29.0\left(\mathrm{CH}_{2}\right), 25.8\left(\mathrm{CH}_{2}\right), 24.2\left(\mathrm{CH}_{3}\right) 21.5\left(\mathrm{CH}_{2}\right) \mathrm{ppm}$; HRMS (El) m/z: [M+] calc'd for $\mathrm{C}_{15} \mathrm{H}_{20} \mathrm{BrNO}_{2} \mathrm{~S} 357.0398$; Found 357.03911.

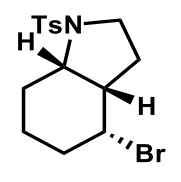

(3aS,7aS)-4-bromo-1-tosyloctahydro-1H-indole (2r')

Synthesized according to GP5. Crude mixture was purified by flash chromatography $(0-100 \%$ EtOAc:Hexane) where relevant fractions were combined. Isolated as $21 \mathrm{mg}$ of a yellow oil as a $1: 1$ mixture of diastereoisomers (29\%). Characterized according to NMR comparaison. ${ }^{3}$

${ }^{1} \mathrm{H}$ NMR $\left(400 \mathrm{MHz}, \mathrm{CDCl}_{3}\right) \delta=7.73-7.67(\mathrm{~m}, 2 \mathrm{H}), 7.36-7.29(\mathrm{~m}, 2 \mathrm{H}), 4.20(\mathrm{td}, J=7.0,3.8 \mathrm{~Hz}$, $1 \mathrm{H}), 3.70-3.62(\mathrm{~m}, 1 \mathrm{H}), 3.54(\mathrm{ddd}, J=10.7,7.9,6.0 \mathrm{~Hz}, 1 \mathrm{H}), 3.27$ (ddd, $J=10.7,8.7,6.0 \mathrm{~Hz}$, $1 \mathrm{H}), 2.43(\mathrm{~s}, 3 \mathrm{H}), 2.20(\mathrm{p}, J=6.4 \mathrm{~Hz}, 1 \mathrm{H}), 2.11-1.97(\mathrm{~m}, 2 \mathrm{H}), 1.96-1.58(\mathrm{~m}, 6 \mathrm{H}) \mathrm{ppm} ;{ }^{13} \mathrm{C} \mathrm{NMR}$ $\left(101 \mathrm{MHz}, \mathrm{CDCl}_{3}\right) \delta=143.5(\mathrm{C}), 134.1(\mathrm{C}), 129.7(2 \mathrm{X} \mathrm{CH}), 127.4(2 \mathrm{X} \mathrm{CH}), 59.0(\mathrm{CH}), 52.9(\mathrm{CH})$, $48.1(\mathrm{CH}), 47.2\left(\mathrm{CH}_{2}\right), 33.1\left(\mathrm{CH}_{2}\right), 28.6\left(\mathrm{CH}_{2}\right), 27.7\left(\mathrm{CH}_{2}\right), 21.5\left(\mathrm{CH}_{3}\right), 20.3\left(\mathrm{CH}_{2}\right)$ ppm; HRMS (EI) $\mathrm{m} / \mathrm{z}:\left[\mathrm{M}^{+}\right]$calc'd for $\mathrm{C}_{15} \mathrm{H}_{20} \mathrm{BrNO}_{2} \mathrm{~S} 357.0398$; Found 357.03911.

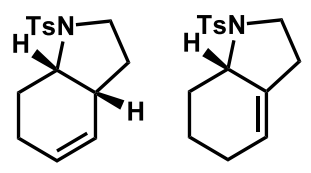

(3aR,7aS)-1-tosyl-2,3,3a,6,7,7a-hexahydro-1H-indole (2r')

Synthesized according to GP5. Crude mixture was purified by flash chromatography $(0-100 \%$ EtOAc:Hexane) where relevant fractions were combined. Isolated as $21 \mathrm{mg}$ of a yellow oil as a $1: 1$ mixture of $2 r$ and $2 r "$ " $(29 \%)$.

${ }^{1} \mathrm{H}$ NMR $\left(400 \mathrm{MHz}, \mathrm{CDCl}_{3}\right) \delta=7.73-7.65(\mathrm{~m}, 2 \mathrm{H}), 7.32-7.25(\mathrm{~m}, 2 \mathrm{H}), 5.78-5.71(\mathrm{~m}, 1 \mathrm{H}), 5.50$ $-5.42(\mathrm{~m}, 1 \mathrm{H}), 3.70-3.63(\mathrm{~m}, 1 \mathrm{H}), 3.55-3.46(\mathrm{~m}, 1 \mathrm{H}), 3.46-3.39(\mathrm{~m}, 1 \mathrm{H}), 3.16-3.08(\mathrm{~m}$, $1 \mathrm{H}), 2.40(\mathrm{~s}, 3 \mathrm{H}), 2.34-2.23(\mathrm{~m}, 1 \mathrm{H}), 2.14-2.04(\mathrm{~m}, 1 \mathrm{H}), 2.03-1.91(\mathrm{~m}, 1 \mathrm{H}), 1.84-1.67(\mathrm{~m}$, $1 \mathrm{H}), 1.61-1.48(\mathrm{~m}, 1 \mathrm{H}), 1.40-1.26(\mathrm{~m}, 1 \mathrm{H}) \mathrm{ppm} ;{ }^{13} \mathrm{C} \mathrm{NMR}\left(101 \mathrm{MHz}, \mathrm{CDCl}_{3}\right) \delta=143.2(\mathrm{C})$, $134.8(\mathrm{C}), 129.6(2 \times \mathrm{CH}), 129.5(\mathrm{CH}), 127.4(2 \times \mathrm{CH}), 127.3(\mathrm{CH}), 58.4(\mathrm{CH}), 47.9\left(\mathrm{CH}_{2}\right), 38.9$ $(\mathrm{CH}), 30.7\left(\mathrm{CH}_{2}\right), 27.20\left(\mathrm{CH}_{2}\right), 22.6\left(\mathrm{CH}_{2}\right), 21.6\left(\mathrm{CH}_{3}\right)$ ppm; HRMS (El) m/z: [M+] calc'd for $\mathrm{C}_{15} \mathrm{H}_{19} \mathrm{NO}_{2} \mathrm{~S} 277.1136$; Found 277.1135.

1-tosyl-2,3,5,6,7,7a-hexahydro-1H-indole (2r')

Synthesized according to GP5. Isolated as $21 \mathrm{mg}$ of a yellow oil as a $1: 1$ mixture of $2 r^{\prime}$ and $2 r^{\prime \prime}$ (29\%).

${ }^{1} \mathrm{H}$ NMR $\left(400 \mathrm{MHz}, \mathrm{CDCl}_{3}\right) \delta=7.73-7.65(\mathrm{~m}, 2 \mathrm{H}), 7.32-7.25(\mathrm{~m}, 2 \mathrm{H}), 5.50-5.42(\mathrm{~m}, 1 \mathrm{H}), 3.70$ $-3.63(\mathrm{~m}, 1 \mathrm{H}), 3.55-3.46(\mathrm{~m}, 1 \mathrm{H}), 3.46-3.39(\mathrm{~m}, 1 \mathrm{H}), 3.16-3.08(\mathrm{~m}, 1 \mathrm{H}), 2.40(\mathrm{~s}, 3 \mathrm{H}), 2.34$

$-2.23(\mathrm{~m}, 1 \mathrm{H}), 2.14-2.04(\mathrm{~m}, 1 \mathrm{H}), 2.03-1.91(\mathrm{~m}, 2 \mathrm{H}), 1.84-1.67(\mathrm{~m}, 1 \mathrm{H}), 1.61-1.48(\mathrm{~m}$, $1 \mathrm{H}), 1.40-1.26(\mathrm{~m}, 1 \mathrm{H}) \mathrm{ppm} ;{ }^{13} \mathrm{C}$ NMR $\left(101 \mathrm{MHz}, \mathrm{CDCl}_{3}\right) \delta=143.3(\mathrm{C}), 143.0(\mathrm{C}), 134.5(\mathrm{C})$, $128.6(2 \times \mathrm{CH}), 126.7(2 \times \mathrm{CH}), 122.1(\mathrm{CH}), 58.7(\mathrm{CH}), 47.1\left(\mathrm{CH}_{2}\right), 29.9\left(\mathrm{CH}_{2}\right), 26.3\left(\mathrm{CH}_{2}\right), 24.3$ $\left(\mathrm{CH}_{2}\right)$, $23.2\left(\mathrm{CH}_{2}\right), 21.6\left(\mathrm{CH}_{3}\right)$ ppm; HRMS (EI) m/z: [ $\left.\mathrm{M}^{+}\right]$calc'd for $\mathrm{C}_{15} \mathrm{H}_{19} \mathrm{NO}_{2} \mathrm{~S} 277.1136$; Found 277.1135. 
<smiles>C[C@]12CCN3[AlH2]CC[C@@]1(Br)[C@H](Br)CCC32</smiles>

(3aS,7aS)-4-bromo-3a-methyl-1-tosyloctahydro-1H-indole (2s)

Synthesized according to GP5. Crude mixture was purified by flash chromatography $(0-100 \%$ EtOAc:Hexane) where relevant fractions were combined. Isolated as $31 \mathrm{mg}$ of a yellow oil as a 2:1 mixture of diastereoisomers (41\%).

${ }^{1} \mathrm{H}$ NMR $\left(400 \mathrm{MHz}, \mathrm{CDCl}_{3}\right) \delta=7.74-7.69(\mathrm{~m}, 2 \mathrm{H}$, major diasteroisomer $), 7.69-7.65(\mathrm{~m}, 1 \mathrm{H}$, minor diastereoisomer), $7.35-7.32(\mathrm{~m}, 1 \mathrm{H}$, minor diastereoisomer $), 7.32-7.27(\mathrm{~m}, 2 \mathrm{H}$, major diastereoisomer), $4.27(\mathrm{dd}, J=11.9,4.2 \mathrm{~Hz}, 0.5 \mathrm{H}$, minor diastereoisomer), 3.94 (dd, $J$ $=12.6,4.8 \mathrm{~Hz}, 1 \mathrm{H}$, major diastereoisomer $), 3.59-3.53(\mathrm{~m}, 0.5 \mathrm{H}$, minor diastereoisomer $)$, $3.53-3.45(\mathrm{~m}, 1 \mathrm{H}$, major diastereoisomer), $3.39(\mathrm{dt}, J=10.6,5.2 \mathrm{~Hz}, 1 \mathrm{H}$, major diastereoisomer), $3.36-3.26(\mathrm{~m}, 0.5 \mathrm{H}$, minor diastereoisomer), $3.19(\mathrm{td}, J=10.0,7.7 \mathrm{~Hz}, 1 \mathrm{H}$, major diastereoisomer), $2.99(\mathrm{~d}, J=3.4 \mathrm{~Hz}, 0.5 \mathrm{H}$, minor diastereoisomer), $2.44(\mathrm{~s}, 1.5 \mathrm{H}$, minor diastereoisomer), $2.42(\mathrm{~s}, 3 \mathrm{H}$, major diastereoisomer $), 2.19-2.08(\mathrm{~m}, 2 \mathrm{H}$, major diastereoisomer), $2.19-2.08(\mathrm{~m}, 1 \mathrm{H}$, minor diastereoisomers) $1.95-1.77(\mathrm{~m}, 2 \mathrm{H}), 1.70-$ $1.58(\mathrm{~m}, 2 \mathrm{H}$, major diastereoisomer), $1.70-1.58(\mathrm{~m}, 1 \mathrm{H}$, major diastereoisomers) $1.44-1.30(\mathrm{~m}$, $2 \mathrm{H}), 1.24-1.09(\mathrm{~m}, 2 \mathrm{H}), 1.06(\mathrm{~s}, 1.5 \mathrm{H}$, minor diastereoisomer $), 0.70(\mathrm{~s}, 3 \mathrm{H}$, major diastereoisomer) ppm; ${ }^{13} \mathrm{C}$ NMR $\left(101 \mathrm{MHz} \mathrm{CDCl}_{3}\right) \delta=143.6(\mathrm{C}$, minor diastereoisomer), 143.2 (C, major diastereoisomer), 135.9 (C, major diastereoisomer), 133.4 (C, minor diastereoisomer), $129.7(2 \times \mathrm{CH}$, minor diastereoisomer $), 129.59$ (2 X CH, major diastereoisomer), 127.6 ( $2 \mathrm{X} \mathrm{CH}$, minor diastereoisomer), $66.2(\mathrm{CH}$, minor diastereoisomer), $65.4(\mathrm{CH}$, major diastereoisomer), $60.0(\mathrm{CH}$, major diastereoisomer $), 58.5(\mathrm{CH}$, minor diastereoisomer), $47.0\left(\mathrm{CH}_{2}\right.$, major diastereoisomer $), 46.8\left(\mathrm{CH}_{2}\right.$, minor diastereoisomer), $46.6\left(\mathrm{CH}_{2}\right.$, minor diastereoisomer $), 44.0\left(\mathrm{CH}_{2}\right.$, major diastereoisomer $), 35.0\left(\mathrm{CH}_{2}\right.$, minor diastereoisomer), $33.8\left(\mathrm{CH}_{2}\right.$, major diastereoisomer), $33.3\left(\mathrm{CH}_{2}\right.$, minor diastereoisomer), $31.6\left(\mathrm{CH}_{2}\right.$, major diastereoisomer), $31.1\left(\mathrm{CH}_{2}\right.$, major diastereoisomer $), 25.46\left(\mathrm{CH}_{3}\right) 24.86$ $\left(\mathrm{CH}_{2}\right.$, minor diastereoisomer $), 24.2\left(\mathrm{CH}_{2}\right.$, major diastereoisomer $), 21.6\left(\mathrm{CH}_{3}\right.$, minor diastereoisomer), $21.5\left(\mathrm{CH}_{3}\right.$, major diastereoisomer), 20.1 (C) ppm; HRMS (EI) m/z: [M+] calc'd for $\mathrm{C}_{16} \mathrm{H}_{22} \mathrm{BrNO}_{2} \mathrm{~S} 371.0555$; Found 371.0565 .

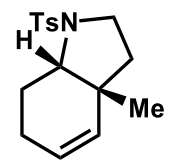

(3aR,7aS)-3a-methyl-1-tosyl-2,3,3a,6,7,7a-hexahydro-1H-indole (2s')

Synthesized according to GP5. Crude mixture was purified by flash chromatography (0-100\% EtOAc:Hexane) where relevant fractions were combined. Isolated as $31 \mathrm{mg}$ of a yellow oil (58\%). ${ }^{1} \mathbf{H}$ NMR $\left(400 \mathrm{MHz}, \mathrm{CDCl}_{3}\right) \delta=7.75-7.69(\mathrm{~m}, 2 \mathrm{H}), 7.33-7.27(\mathrm{~m}, 2 \mathrm{H}), 5.71(\mathrm{dt}, \mathrm{J}=10.0,3.8$ $\mathrm{Hz}, 1 \mathrm{H}), 5.31-5.24(\mathrm{~m}, 1 \mathrm{H}), 3.35-3.26(\mathrm{~m}, 3 \mathrm{H}), 2.42(\mathrm{~s}, 3 \mathrm{H}), 2.21-2.11(\mathrm{~m}, 1 \mathrm{H}), 2.02-1.79$ $(\mathrm{m}, 4 \mathrm{H}), 1.59$ (ddd, $J=12.0,6.5,5.3 \mathrm{~Hz}, 1 \mathrm{H}), 0.74(\mathrm{~s}, 3 \mathrm{H}) \mathrm{ppm} ;{ }^{13} \mathrm{C} \mathbf{N M R}\left(101 \mathrm{MHz}, \mathrm{CDCl}_{3}\right) \delta=$ $143.1(\mathrm{C}), 134.9(\mathrm{C}), 132.4(\mathrm{CH}), 129.6(2 \times \mathrm{CH}), 127.4(2 \times \mathrm{CH}), 127.3(\mathrm{CH}), 64.5(\mathrm{CH}), 47.1$ $\left(\mathrm{CH}_{2}\right), 37.5\left(\mathrm{CH}_{2}\right), 26.2\left(\mathrm{CH}_{2}\right), 25.4\left(\mathrm{CH}_{3}\right), 21.5\left(\mathrm{CH}_{3}\right), 21.3\left(\mathrm{CH}_{2}\right) \mathrm{ppm}$; HRMS (EI) m/z: [M+] calc'd for $\mathrm{C}_{16} \mathrm{H}_{21}$ BrNOS 291.1293; Found 291.1300. 


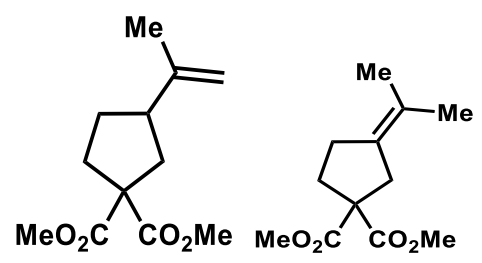

Dimethyl-3-(prop-1-en-2-yl)cyclopentane-1,1-dicarboxylate (2t)

Synthesized according to GP5. Crude mixture was purified by flash chromatography $(0-100 \%$ EtOAc:Hexane) where relevant fractions were combined. Isolated as $34 \mathrm{mg}$ of a yellow oil $(75 \%)$ in a 2:1 mixture of $2 \mathrm{c}$ and $2 c^{\prime}$.

IR (neat, $\left.\mathrm{cm}^{-1}\right)$ : 2954 (m), 2873 (m), 1731 (vs), 1646 (m), 1435 (s), 1247 (s), 1215 (s), 1195 (s), $1155(\mathrm{~s}), 1099(\mathrm{~m}), 1065(\mathrm{~m}), 888(\mathrm{~m}) ;{ }^{1} \mathbf{H}$ NMR (400 MHz, $\left.\mathrm{CDCl}_{3}\right) \delta=4.73-4.67(\mathrm{~m}, 2 \mathrm{H}), 3.71$ $(\mathrm{s}, 6 \mathrm{H}), 2.61-2.51(\mathrm{~m}, 1 \mathrm{H}), 2.48(\mathrm{ddd}, J=12.7,7.2,1.7 \mathrm{~Hz}, 1 \mathrm{H}), 2.40-2.28(\mathrm{~m}, 2 \mathrm{H}), 2.21-$ $2.13(\mathrm{~m}, 1 \mathrm{H}), 1.97(\mathrm{dd}, J=12.9,10.9 \mathrm{~Hz}, 1 \mathrm{H}), 1.92-1.83(\mathrm{~m}, 1 \mathrm{H}), 1.71(\mathrm{~s}, 3 \mathrm{H}) \mathrm{ppm} ;{ }^{13} \mathrm{C}$ NMR $\left(101 \mathrm{MHz}, \mathrm{CDCl}_{3}\right) \delta=173.0(\mathrm{C}), 172.9(\mathrm{C}), 146.5(\mathrm{C}), 109.3\left(\mathrm{CH}_{2}\right), 59.6(\mathrm{C}), 52.6\left(\mathrm{CH}_{3}\right), 52.6$ $\left(\mathrm{CH}_{3}\right), 46.4(\mathrm{CH}), 39.0\left(\mathrm{CH}_{2}\right), 33.7\left(\mathrm{CH}_{2}\right), 30.6\left(\mathrm{CH}_{2}\right), 21.0\left(\mathrm{CH}_{3}\right) \mathrm{ppm} ; \mathrm{HRMS}(\mathrm{ESI}) \mathrm{m} / \mathrm{z}:\left[\mathrm{M}^{+}+\mathrm{Na}^{+}\right]$ calc'd for $\mathrm{C}_{12} \mathrm{H}_{18} \mathrm{O}_{4} \mathrm{Na} 249.1097$; Found 249.1103.

\section{Dimethyl-3-(propan-2-ylidene)cyclopentane-1,1-dicarboxylate (2t')}

Synthesized according to GP5. Crude mixture was purified by flash chromatography (0-100\% EtOAc:Hexane) where relevant fractions were combined. Isolated as $34 \mathrm{mg}$ of a yellow oil $(75 \%)$ in a 2:1 mixture of 2c and 2c'.

IR (neat, $\mathrm{cm}^{-1}$ ): 2954 (m), 2873 (m), 1731 (vs), 1646 (m), 1435 (s), 1247 (s), 1215 (s), 1195 )s), $1155(\mathrm{~s}), 1099(\mathrm{~m}), 1065(\mathrm{~m}), 888(\mathrm{~m}) ;{ }^{1} \mathrm{H}$ NMR $\left(400 \mathrm{MHz}, \mathrm{CDCl}_{3}\right) \delta=3.72(\mathrm{~s}, 6 \mathrm{H}), 2.86-2.80$ $(\mathrm{m}, 2 \mathrm{H}), 2.25-2.22(\mathrm{~m}, 2 \mathrm{H}), 1.62-1.57(\mathrm{~m}, 6 \mathrm{H}), 1.56-1.50(\mathrm{~m}, 2 \mathrm{H}) \mathrm{ppm} ;{ }^{13} \mathrm{C} \mathrm{NMR}(101 \mathrm{MHz}$, $\left.\mathrm{CDCl}_{3}\right) \delta=172.4(2 \times \mathrm{C}), 131.0(\mathrm{C}), 123.3(\mathrm{C}), 60.3(\mathrm{C}), 52.6\left(2 \mathrm{X} \mathrm{CH}_{3}\right), 38.4\left(\mathrm{CH}_{2}\right), 34.0\left(\mathrm{CH}_{2}\right)$, $28.9\left(\mathrm{CH}_{2}\right), 21.0\left(\mathrm{CH}_{3}\right), 21.0\left(\mathrm{CH}_{3}\right)$ ppm; HRMS (ESI) m/z: $\left[\mathrm{M}^{+}+\mathrm{Na}^{+}\right]$calc'd for $\mathrm{C}_{12} \mathrm{H}_{18} \mathrm{O}_{4} \mathrm{Na}$ 249.1097; Found 249.1103.<smiles>O=C1CCC[C@H]2CC[C@@H](CBr)[C@H]12</smiles>

(3S, 3aS, 7aS)-3-(bromomethyl)octahydro-4H-inden-4-one (4)

Synthesized according to GP5. Crude mixture was purified by flash chromatography $(0-100 \%$ EtOAc:Hexane) where relevant fractions were combine. Isolated as a mixture of diastereoisomers. (41\%, d.r. 1:1)

${ }^{1} \mathrm{H}$ NMR $\left(400 \mathrm{MHz}, \mathrm{CDCl}_{3}\right) \delta 3.47-3.29(\mathrm{~m}, 2 \mathrm{H}), 2.77-2.66(\mathrm{~m}, 2 \mathrm{H}), 2.66-2.57(\mathrm{~m}, 1 \mathrm{H}), 2.51$ - $2.38(\mathrm{~m}, 1 \mathrm{H}), 2.38-2.26(\mathrm{~m}, 1 \mathrm{H}), 2.19-1.97(\mathrm{~m}, 3 \mathrm{H}), 1.97-1.84(\mathrm{~m}, 3 \mathrm{H}), 1.80-1.67(\mathrm{~m}$, $1 \mathrm{H}), 1.67-1.60(\mathrm{~m}, 1 \mathrm{H}) \mathrm{ppm} ;{ }^{13} \mathrm{C}$ NMR $\left(101 \mathrm{MHz}, \mathrm{CDCl}_{3}\right) \delta=213.2(\mathrm{C}), 50.4(\mathrm{CH}), 39.6\left(\mathrm{CH}_{2}\right)$, $39.4\left(\mathrm{CH}_{2}\right), 36.9(\mathrm{CH}), 31.9(\mathrm{CH}), 30.9\left(\mathrm{CH}_{2}\right), 30.7\left(\mathrm{CH}_{2}\right), 28.1\left(\mathrm{CH}_{2}\right), 21.9\left(\mathrm{CH}_{2}\right)$ ppm ; HRMS (ESI) $\mathrm{m} / \mathrm{z}$ : $\left[\mathrm{M}^{+}\right]$calc'd for $\mathrm{C}_{10} \mathrm{H}_{15} \mathrm{BrO} 230.0306$; Found 230.0280 . 


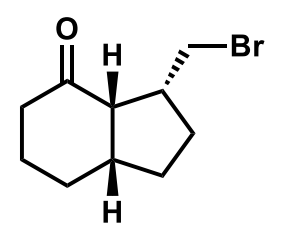

(3R, 3aS, 7aS)-3-(bromomethyl)octahydro-4H-inden-4-one (4')

Synthesized according to GP5. Crude mixture was purified by flash chromatography $(0-100 \%$ EtOAc:Hexane) where relevant fractions were combined. Isolated as a mixture of diastereoisomers. $(41 \%$, d.r. $1: 1)$

${ }^{1} \mathrm{H}$ NMR $\left(400 \mathrm{MHz}, \mathrm{CDCl}_{3}\right) 3.32(\mathrm{t}, J=7.0 \mathrm{~Hz}, 2 \mathrm{H}), 2.77(\mathrm{td}, J=9.3,3.0 \mathrm{~Hz}, 1 \mathrm{H}), 2.71-2.62(\mathrm{~m}$, $1 \mathrm{H}), 2.49-2.33(\mathrm{~m}, 2 \mathrm{H}), 2.33-2.19(\mathrm{~m}, 2 \mathrm{H}), 2.05-1.88(\mathrm{~m}, 4 \mathrm{H}), 1.86-1.77(\mathrm{~m}, 1 \mathrm{H}), 1.77-$ $1.68(\mathrm{~m}, 1 \mathrm{H}), 1.65-1.58(\mathrm{~m}, 2 \mathrm{H}) \mathrm{ppm} ;{ }^{13} \mathrm{C}$ NMR $\left(101 \mathrm{MHz}, \mathrm{CDCl}_{3}\right) \delta=215.1(\mathrm{C}), 43.0(\mathrm{CH}), 42.4$ $(\mathrm{CH}), 41.0\left(\mathrm{CH}_{2}\right), 38.9\left(\mathrm{CH}_{2}\right), 36.4(\mathrm{CH}), 30.9\left(\mathrm{CH}_{2}\right), 29.3\left(\mathrm{CH}_{2}\right), 26.2\left(\mathrm{CH}_{2}\right), 22.3\left(\mathrm{CH}_{2}\right) \mathrm{ppm}$; HRMS (ESI) m/z: [M+] calc'd for $\mathrm{C}_{10} \mathrm{H}_{15} \mathrm{BrO} 230.0306$; Found 230.0280. 


\section{References}

1. Millán, A.; Alvarez de Cienfuegos, L. A.; Miguel, D.; Campaña, A. G.; Cuerva, J. M. Water Control over the Chemoselectivity of a Ti/Ni Multimetallic System: Heck- or ReductiveType Cyclization Reactions of Alkyl lodides. Org. Lett. 2012, 14, 5984-5987.

2. Revol, G.; MCallum, T.; Morin, M.; Gagosz, G.; Barriault, L. Photoredox Transformations via Gold Dimeric Complexes. Angew. Chem. Int. Ed. 2013, 52, 13342-13345.

3. Venning, A. R. O.; Kwiatkowski, M. R.; Roque Peña. J. E.; Lainhart, B. C.; Guruparan, A. A; Alexanian, E. J. Palladium-Catalyzed Carbocyclizations of Unactivated Alkyl Bromides with Alkenes Involving Auto-tandem Catalysis. J. Am. Chem. Soc. 2017, 139, 1159511600.

4. Powell, P. A.; Maki, T.; Fu, G. C. Stille Cross-Couplings of Unactivated Secondary Alkyl Halides Using Monoorganotin Reagents. J. Am. Chem. Soc. 2005, 127, 510-511.

5. Kyne, S. H.; Lévêque C.; Zheng, S.; Fensterbank, L.; Jutand, A.; Ollivier, C. Iron(II) catalyzed reductive radical cyclization reactions of bromoacetals in the presence of $\mathrm{NaBH} 4$ : optimization studies and mechanistic insights. Tetrahedron, 2016, 72, 7727-7737.

6. Hackmann, C; Schaefer, H. J. New methods for reductive free-radical cyclizations of $\alpha-$ bromoacetals to 2-alkoxytetrahydrofurans with activated chromium(II)-acetate. Tetrahedron, 1993, 49, 4559-4574.

7. Zhang, H.; Huang, X. Ligand-Free Heck Reactions of Aryl lodides: Significant Acceleration of the Rate through Visible Light Irradiation at Ambient Temperature Adv. Synth. Catal. 2016, 358, 3736-3742.

8. Gao, Y.; Xiong, W.; Chen, H.; Wu, W.; Peng, J.; Gao, Y.; Jiang, H. Pd-Catalyzed Highly Regio- and Stereoselective Formation of C-C Double Bonds: An Efficient Method for the Synthesis of Benzofuran-, Dihydrobenzofuran-, and Indoline-Containing Alkenes. J. Org. Chem. 2015, 80, 7456-7467.

9. Rixson, J. E.; Chaloner, T.; Heath, C. H.; Tietze, L. F.; Stewart, S. G. The Development of Domino Reactions Incorporating the Heck Reaction: The Formation of N-Heterocycles. Eur. J. Org. Chem. 2012, 3, 544-558.

10. Kim, H.; Lee. C. Visible-light-induced photocatalytic reductive transformations of organohalides. Angew. Chem. Int. Ed. 2012, 51, 12303-12306. 


\section{NMR Spectra}

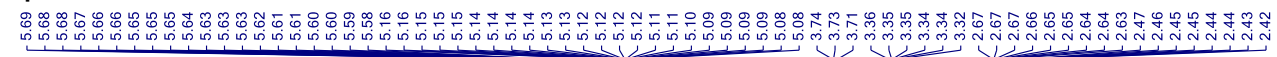

$\mathrm{CDCl}_{3}, 400 \mathrm{MHz}$<smiles>C=CCC(CCBr)(C(=O)OC)C(=O)OC</smiles>

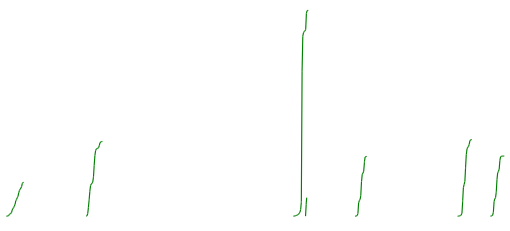

$1 \mathrm{a}$

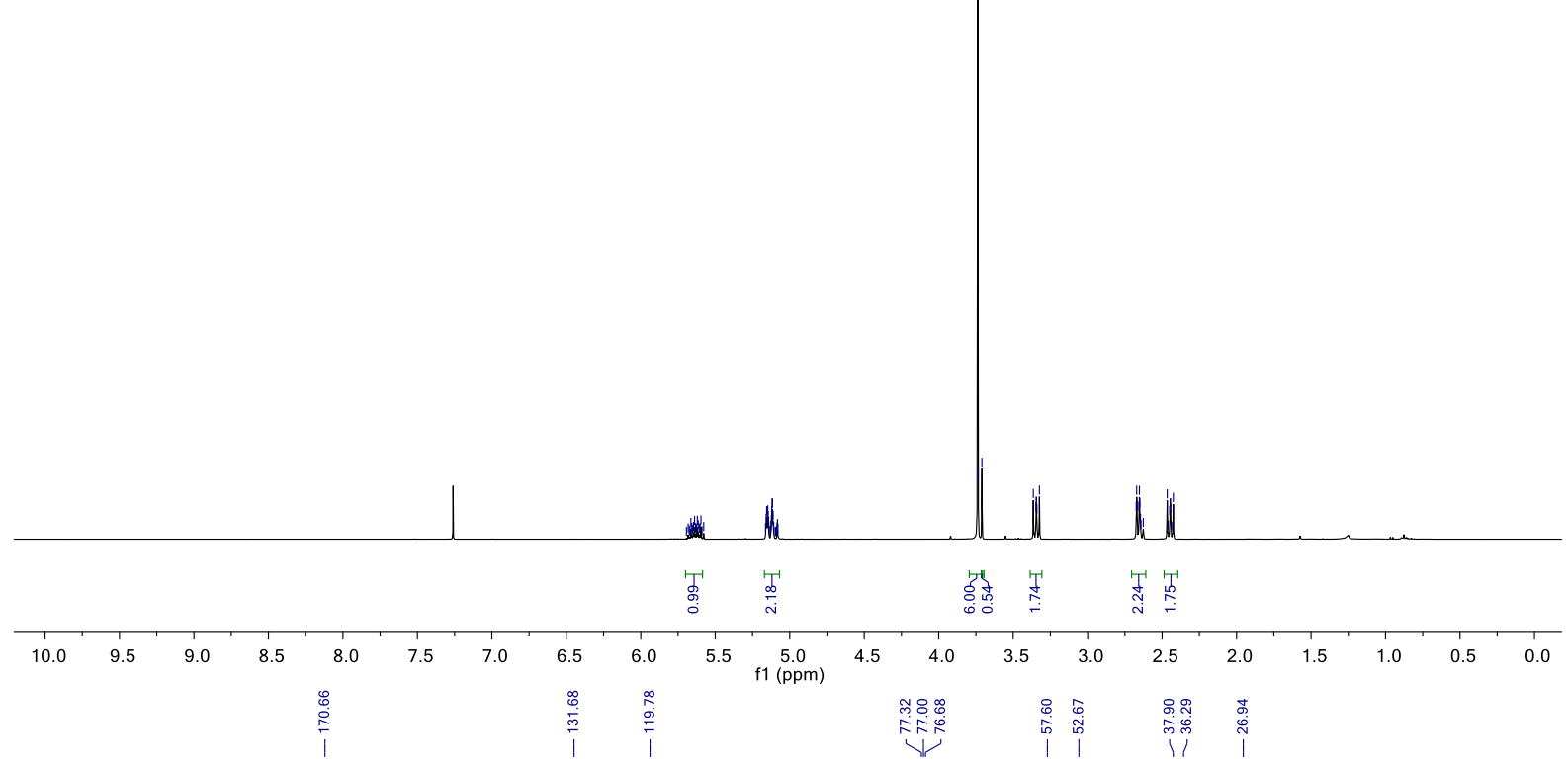

$\mathrm{CDCl}_{3}, 101 \mathrm{MHz}$

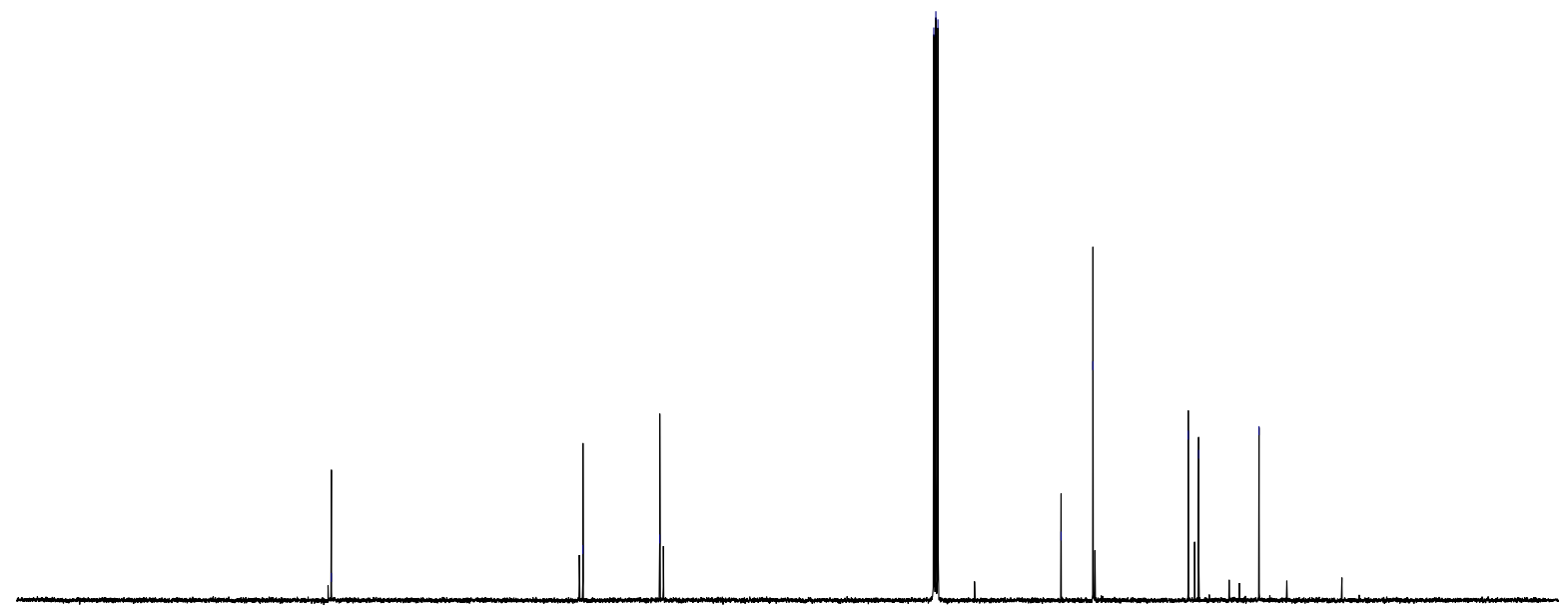

$210 \quad 200 \quad 190 \quad 180$

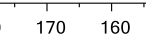

150

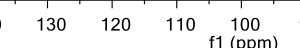

100
$\mathrm{f} 1(\mathrm{ppm})$ 

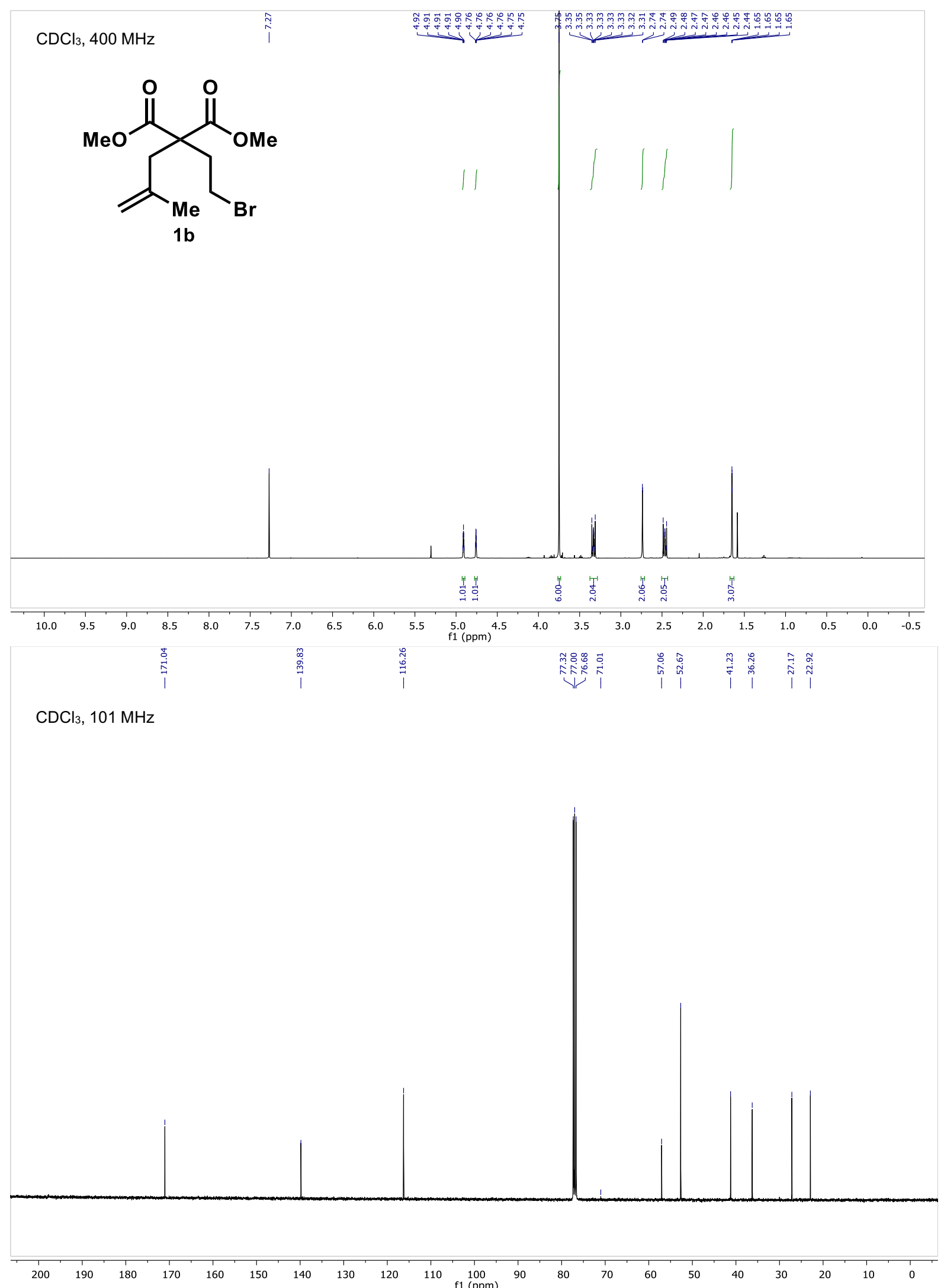


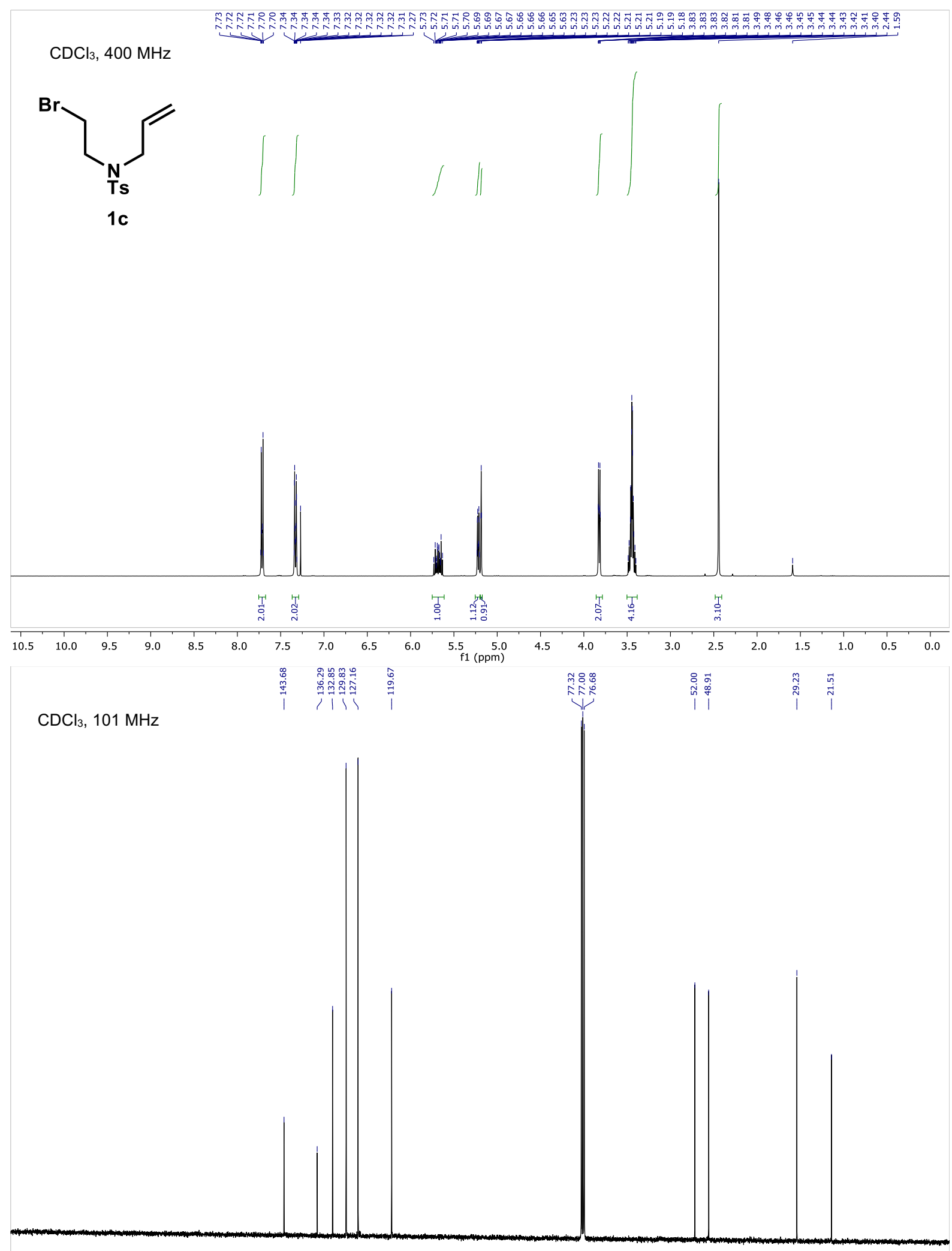

$200 \quad 190 \quad 18$
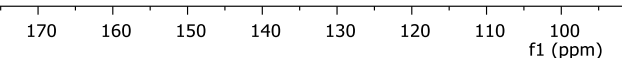

807 

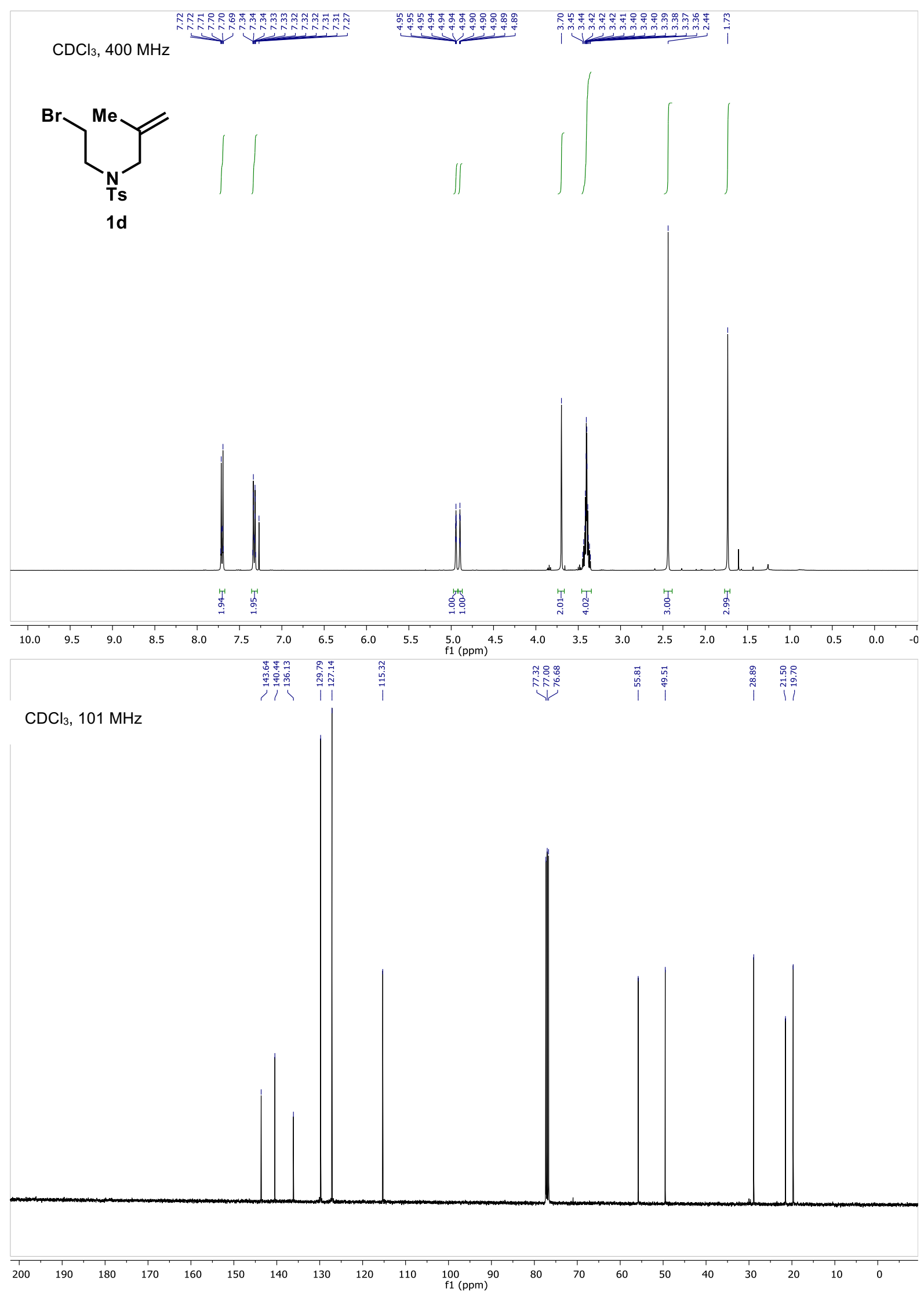


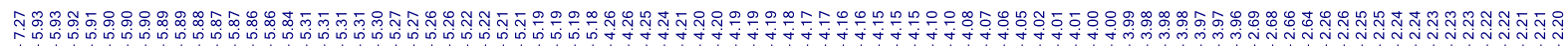

$\mathrm{CDCl}_{3}, 400 \mathrm{MHz}$
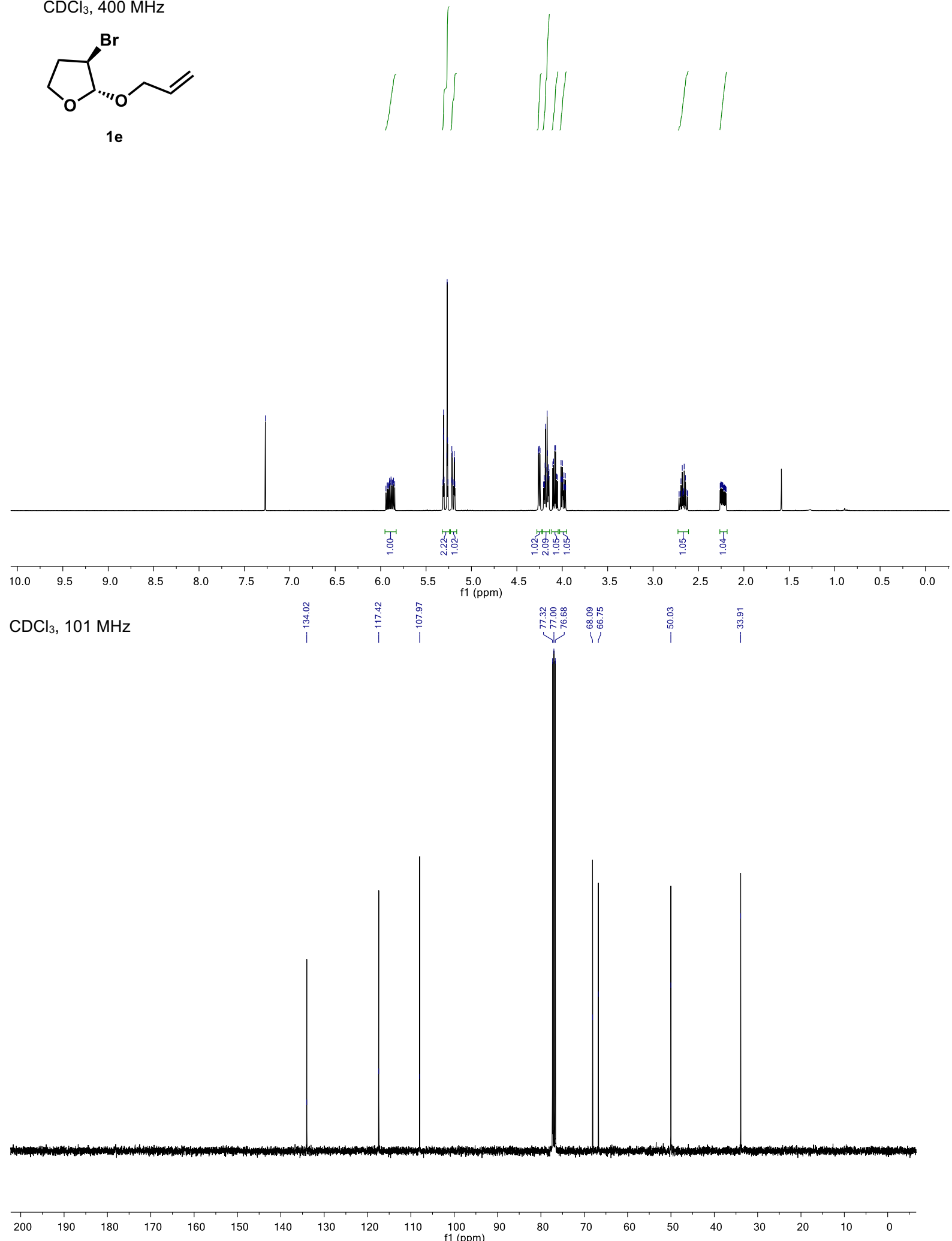
$\mathrm{CDCl}_{3}, 400 \mathrm{MHz}$<smiles>C=CCO[C@H]1OCCC[C@H]1Br</smiles>
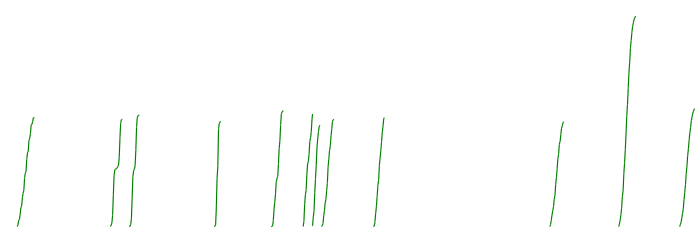

$1 f$
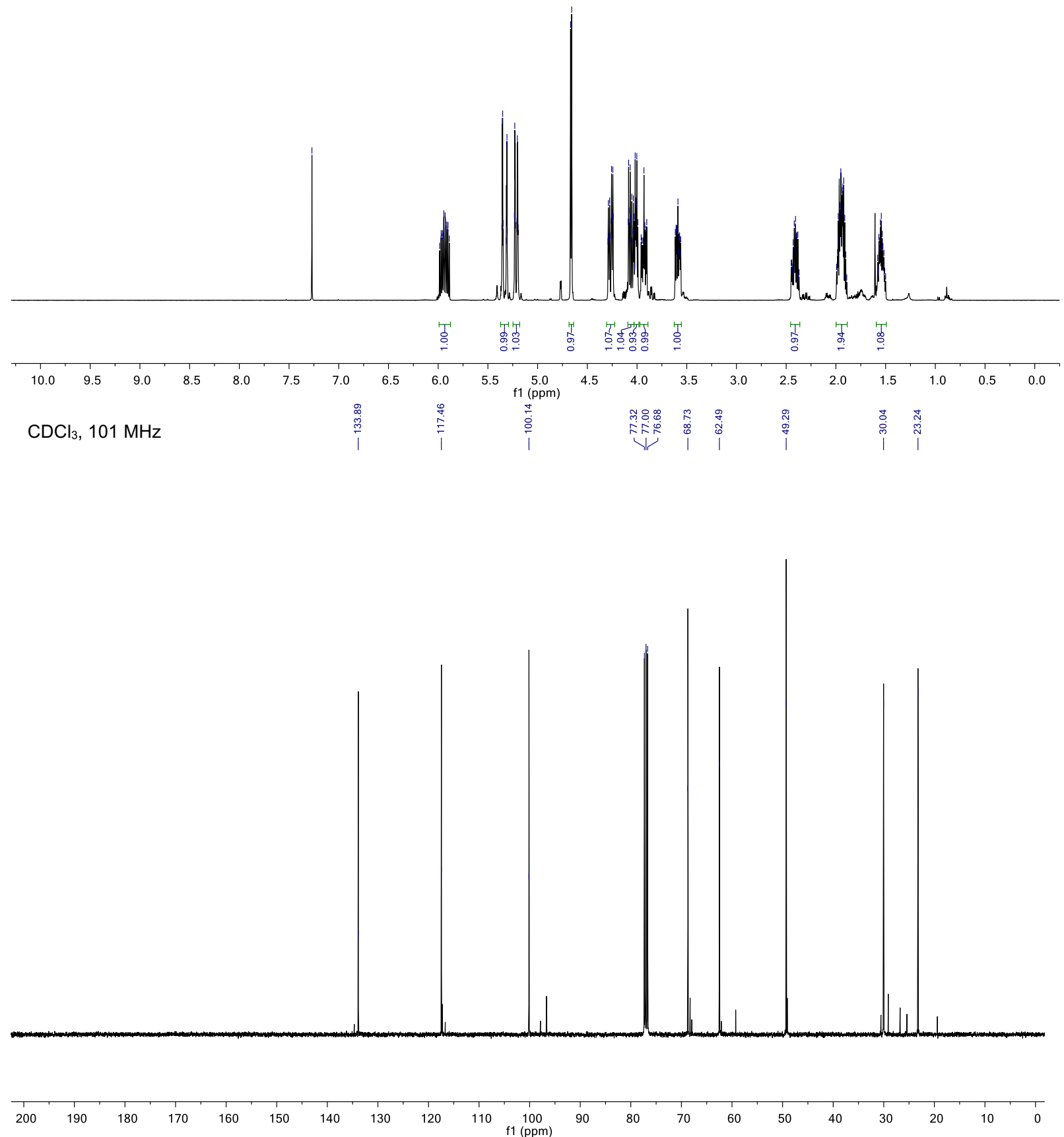


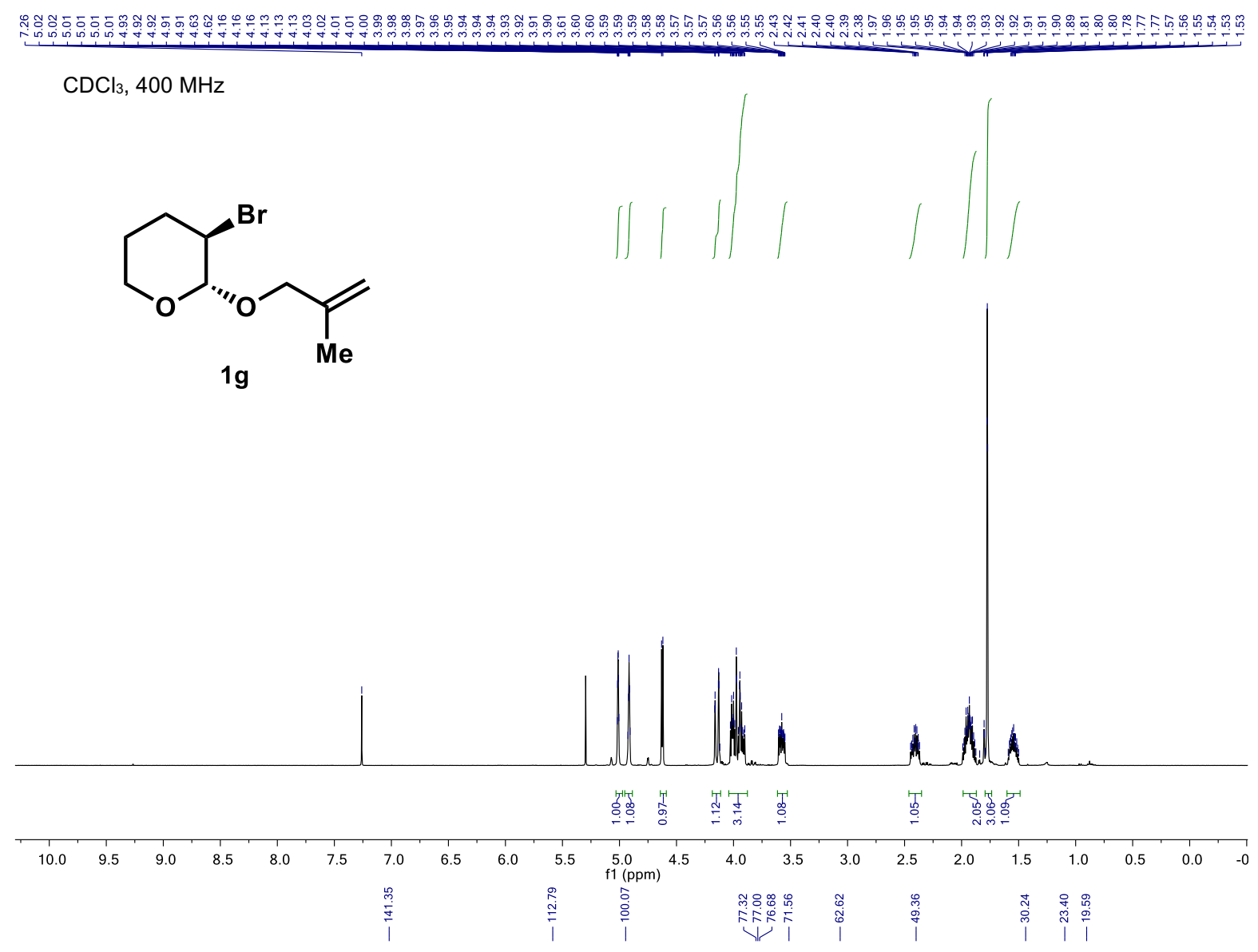

$\mathrm{CDCl}_{3}, 101 \mathrm{MHz}$

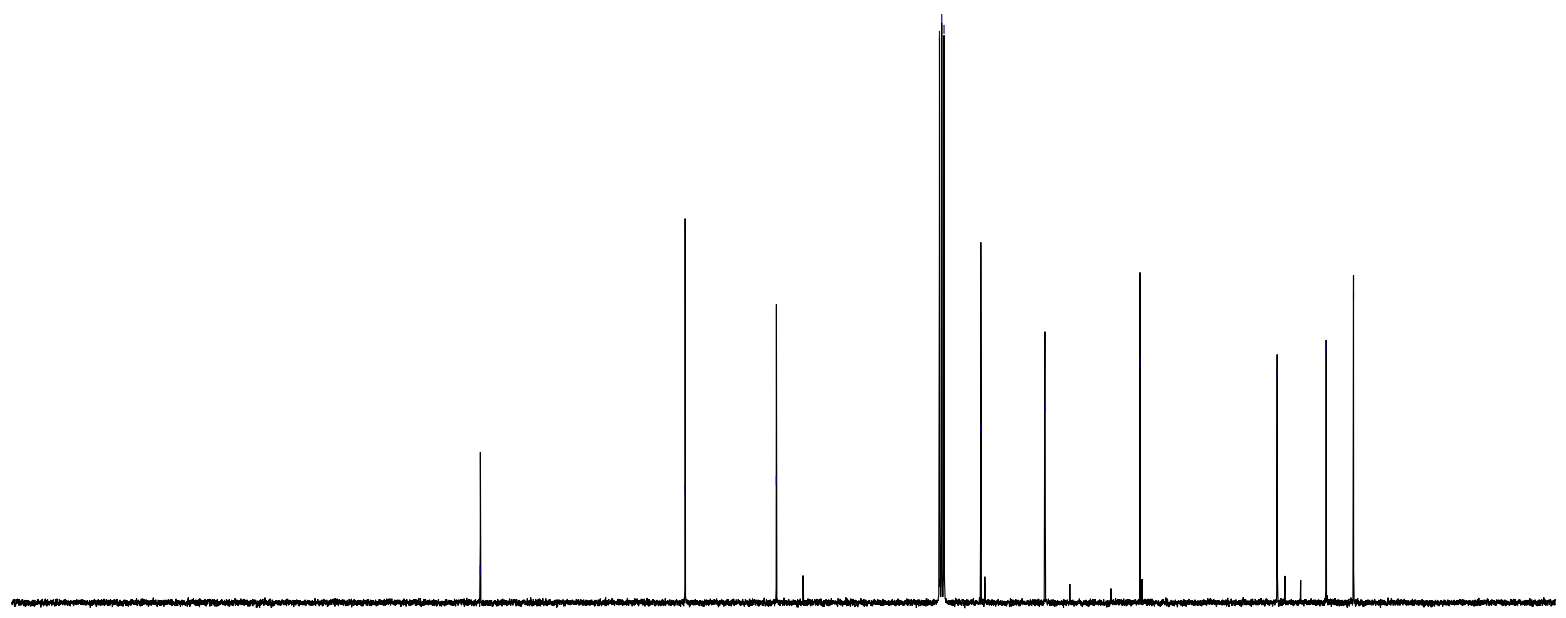

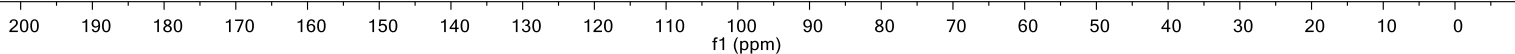




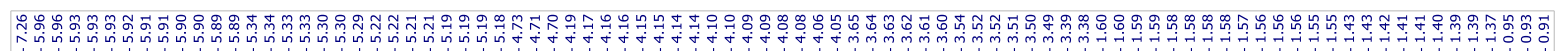

$\mathrm{CDCl}_{3}, 400 \mathrm{MHz}$<smiles>C=CCOC(CBr)OCCC</smiles>

$1 \mathrm{~h}$
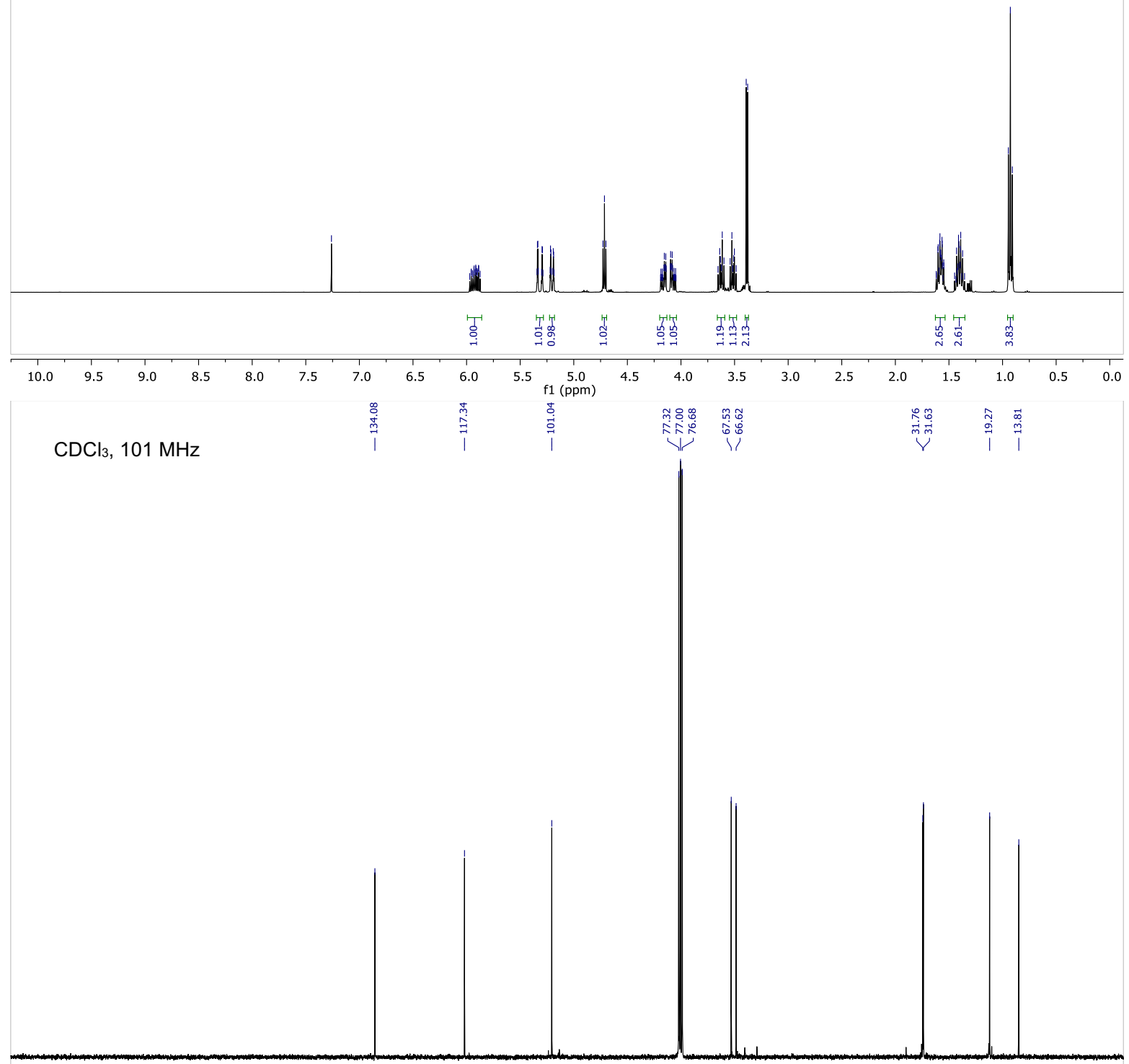

To0

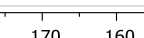

$100 \quad 90$

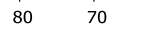



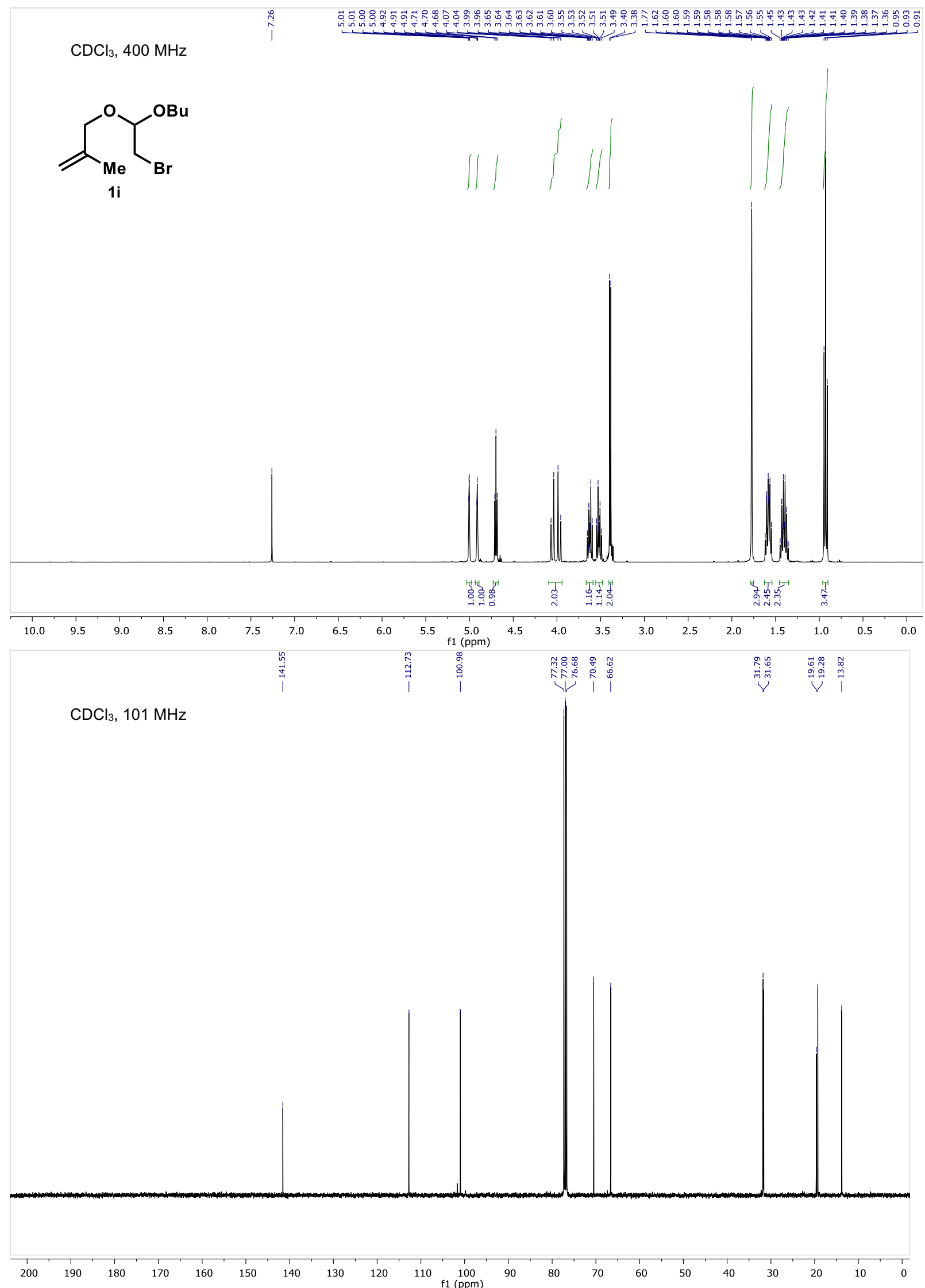
$\mathrm{CDCl}_{3}, 400 \mathrm{MHz}$<smiles>C=CCOc1ccccc1I</smiles>
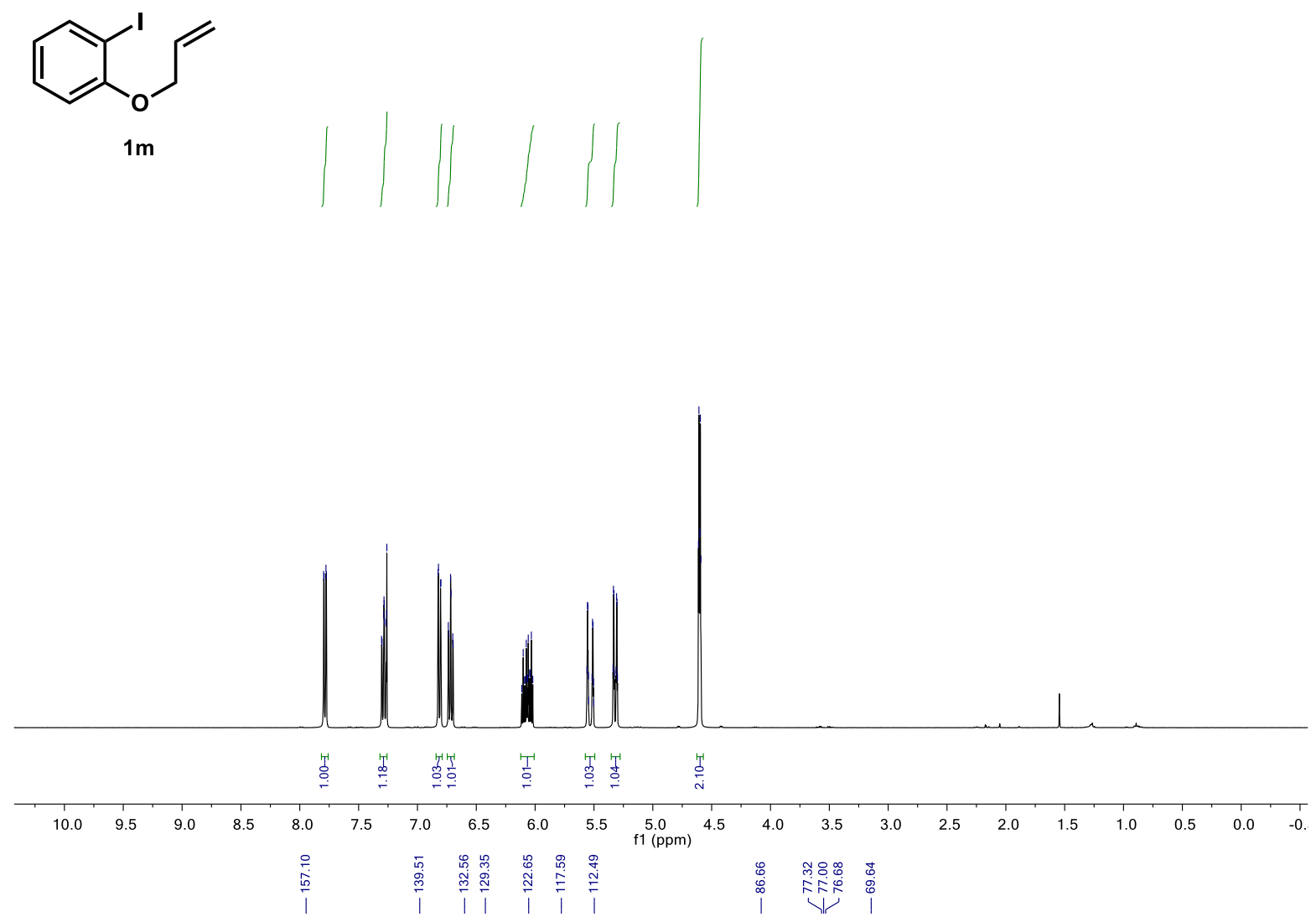

$\mathrm{CDCl}_{3}, 101 \mathrm{MHz}$
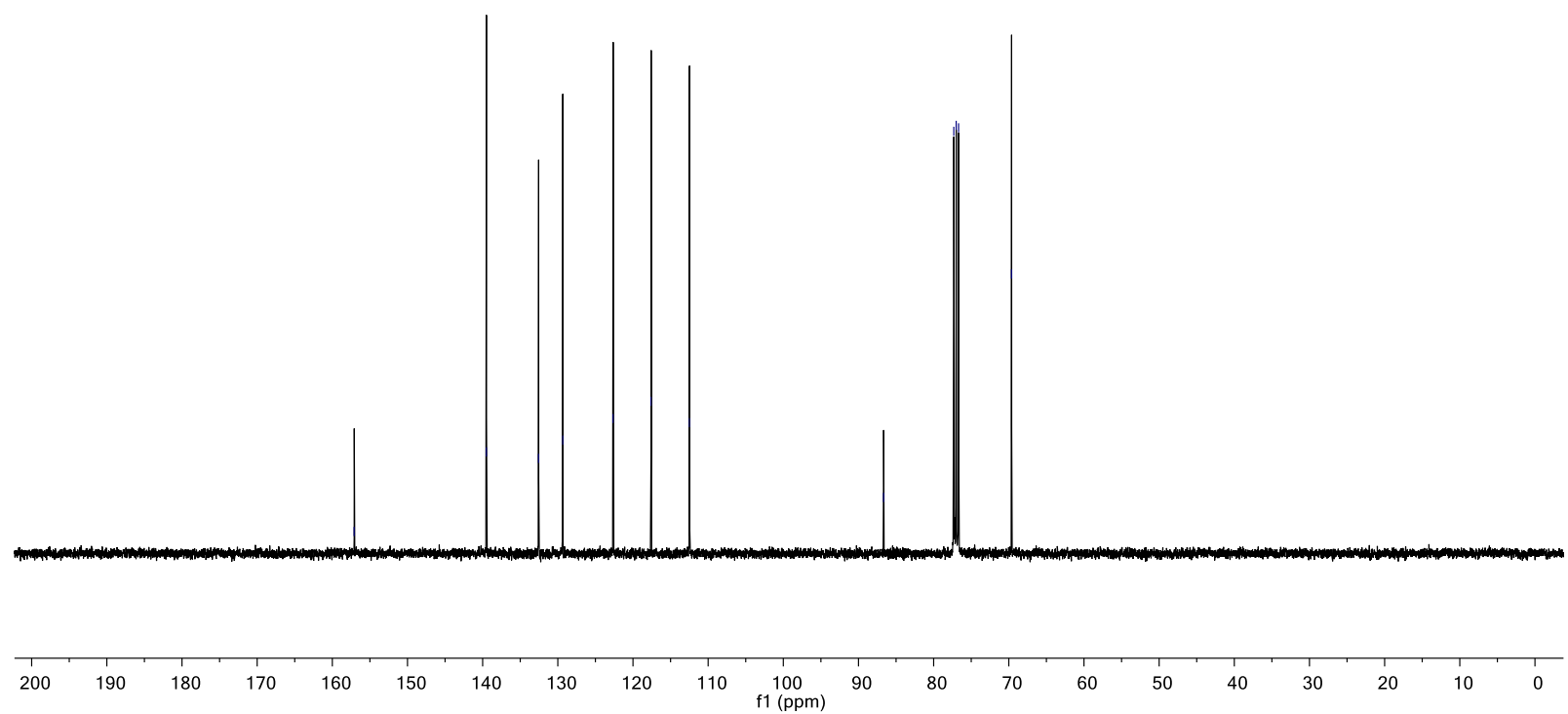


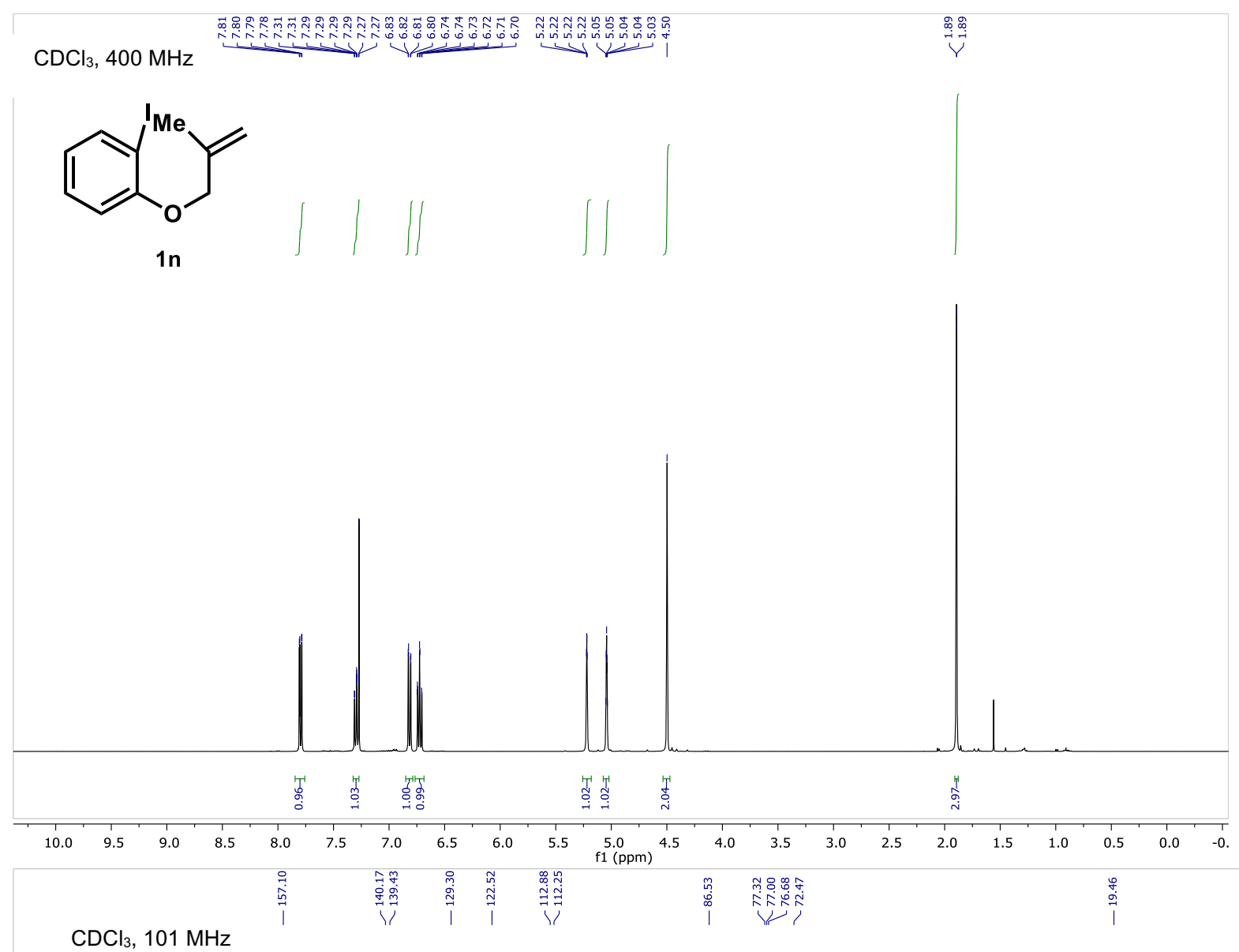

$\mathrm{CDCl}_{3}, 101 \mathrm{MHz}$

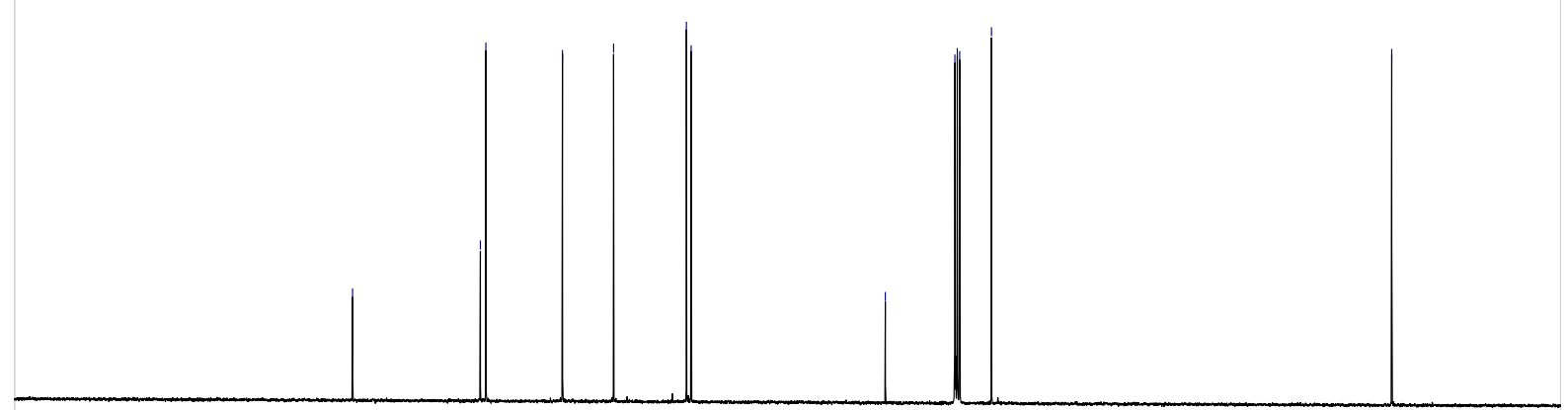




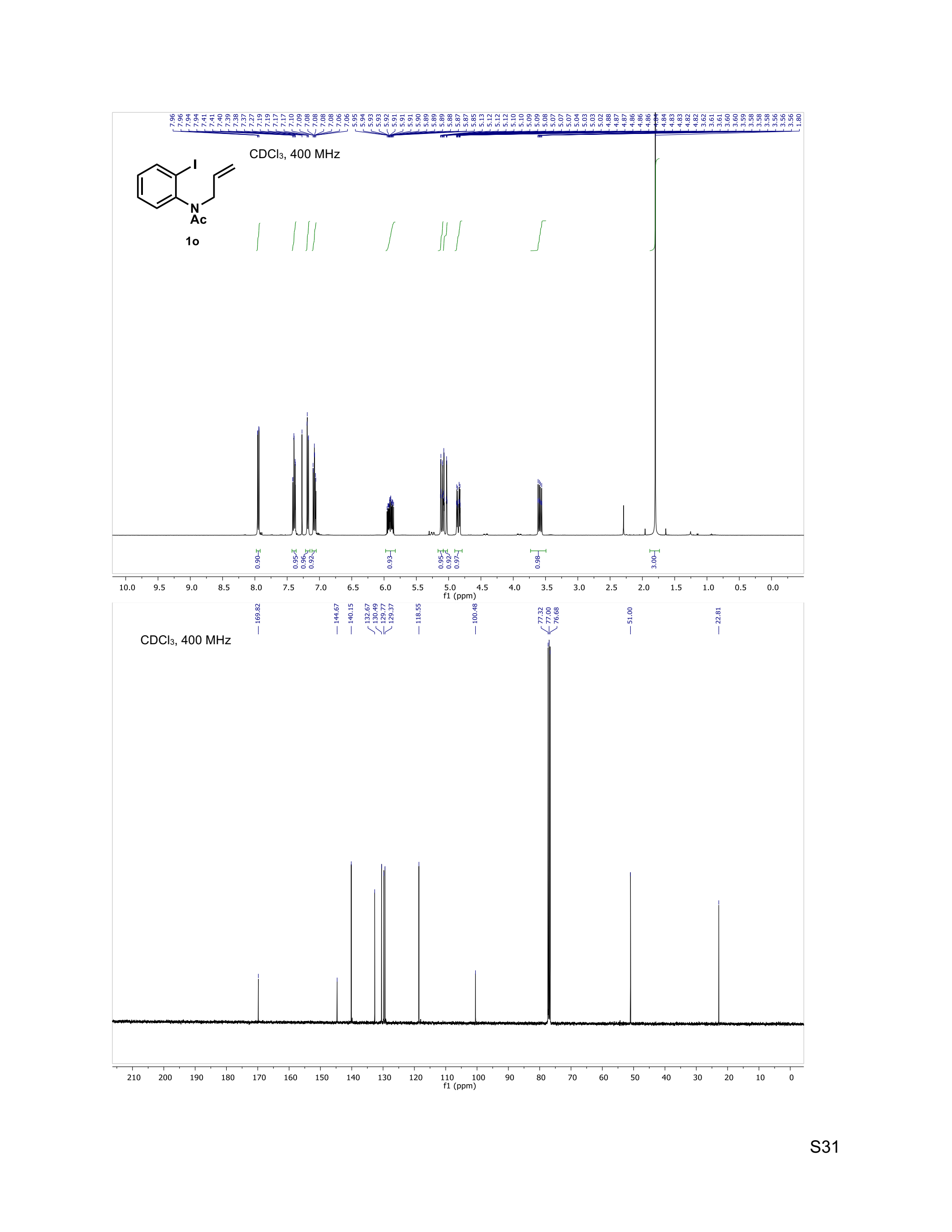


$\mathrm{CDCl}_{3}, 400 \mathrm{MHz}$<smiles>C=CCN(C)c1ccccc1I</smiles>

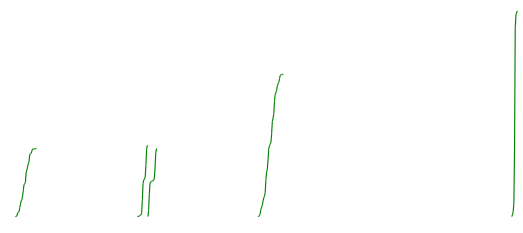

$1 p$
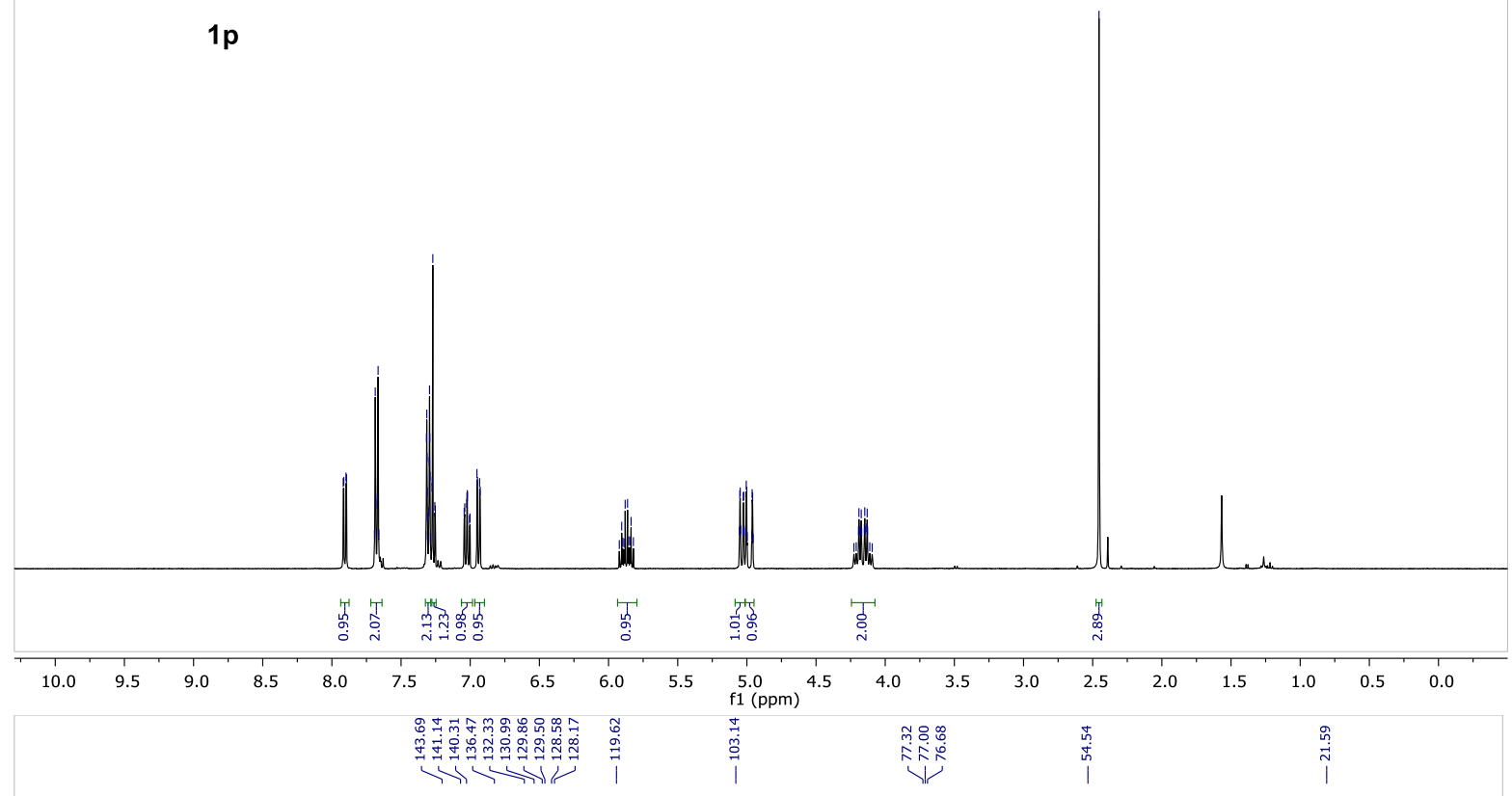

$\mathrm{CDCl}_{3}, 101 \mathrm{MHz}$
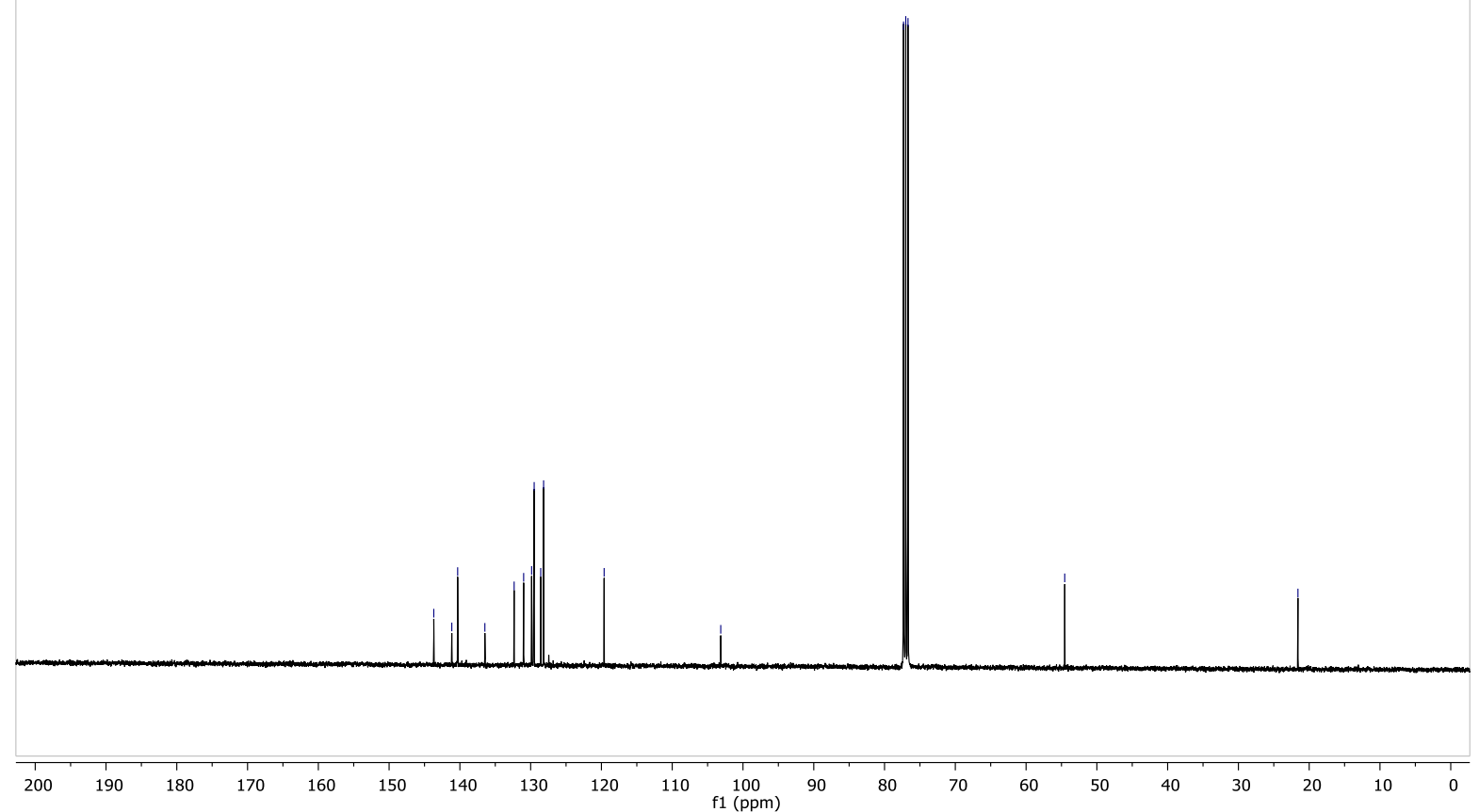


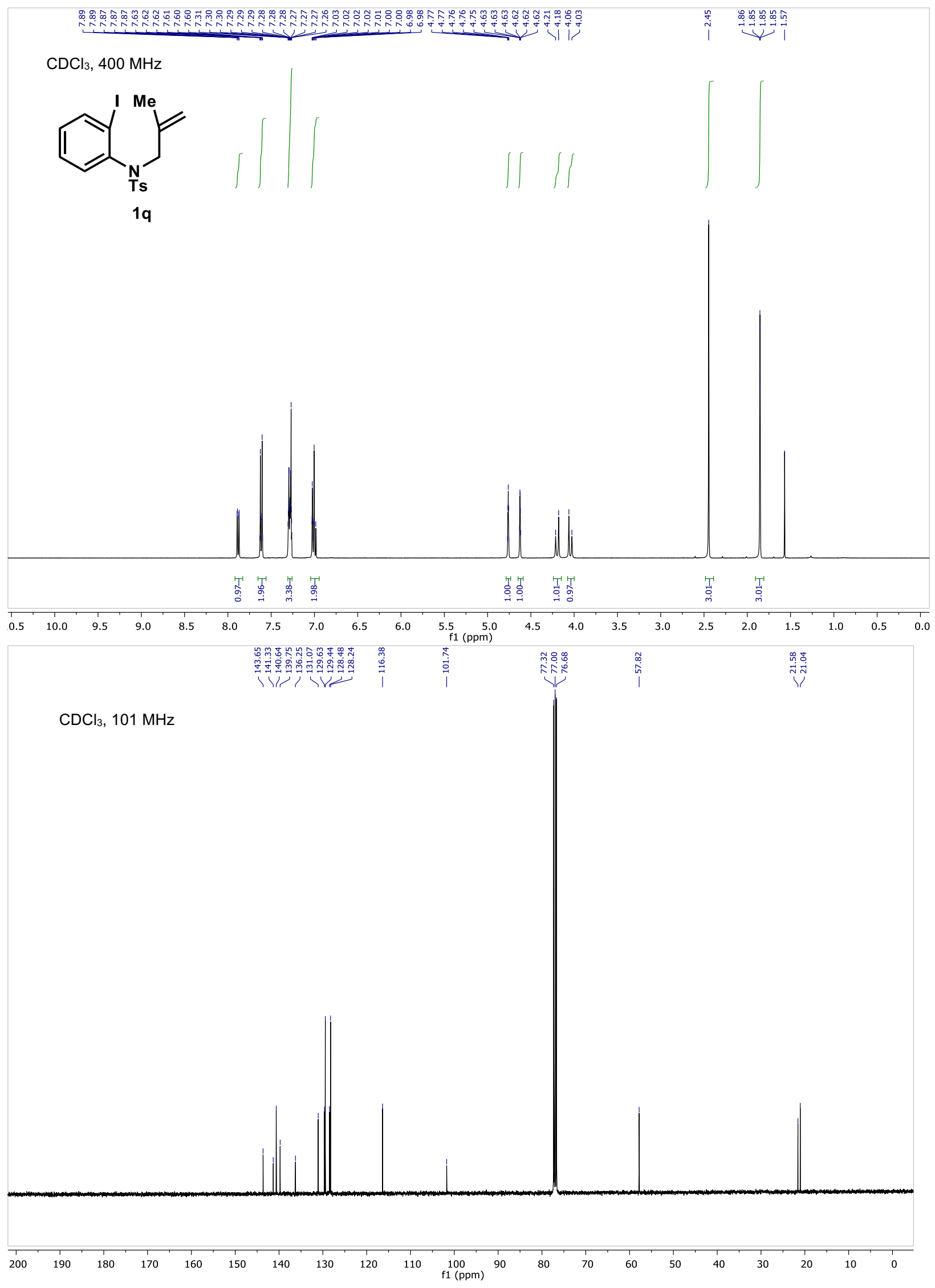

S33 


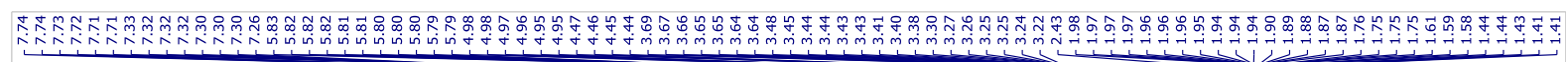

$\mathrm{CDCl}_{3}, 400 \mathrm{MHz}$
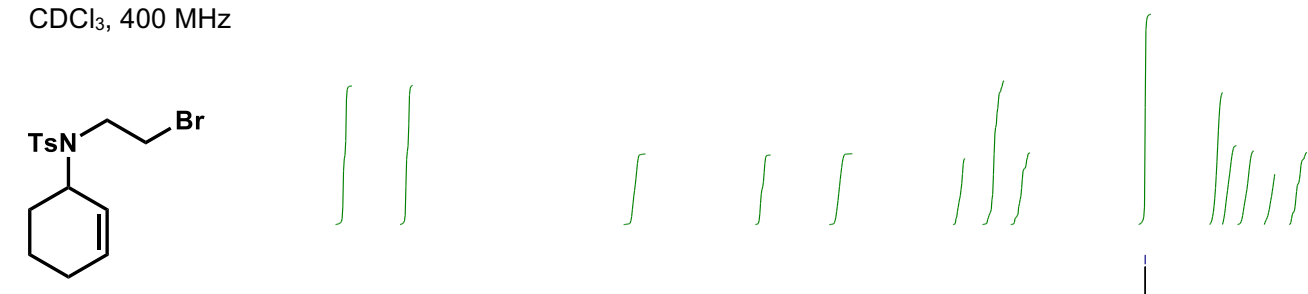

$1 \mathrm{r}$

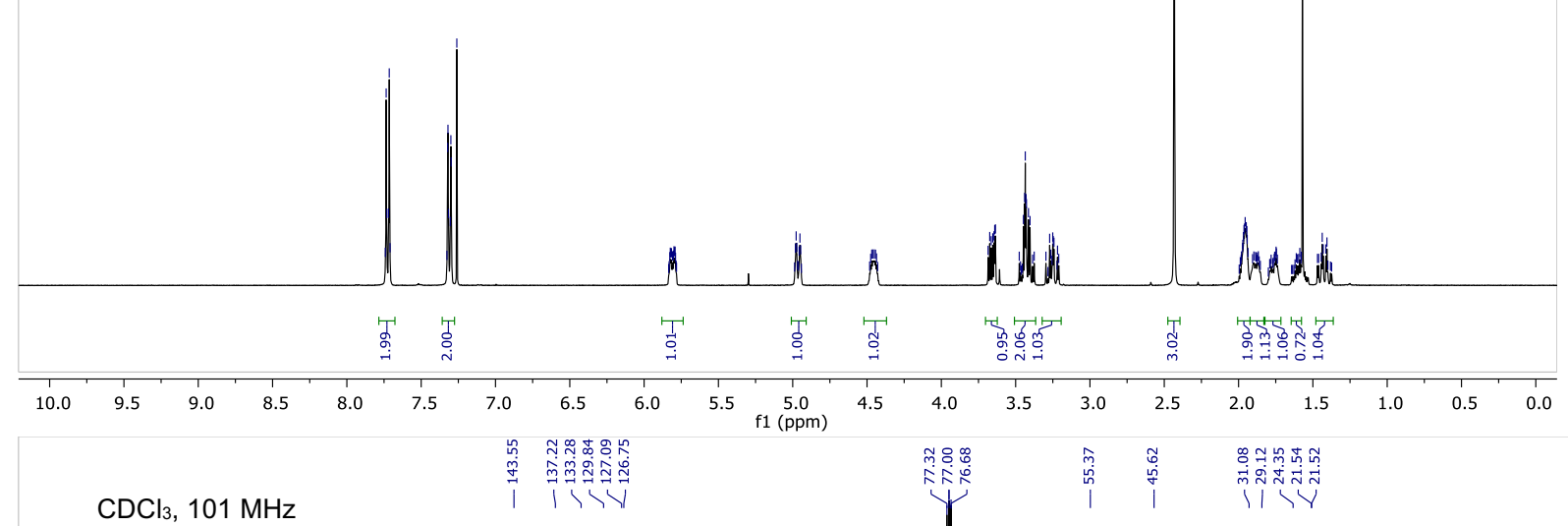

$\mathrm{CDCl}_{3}, 101 \mathrm{MHz}$

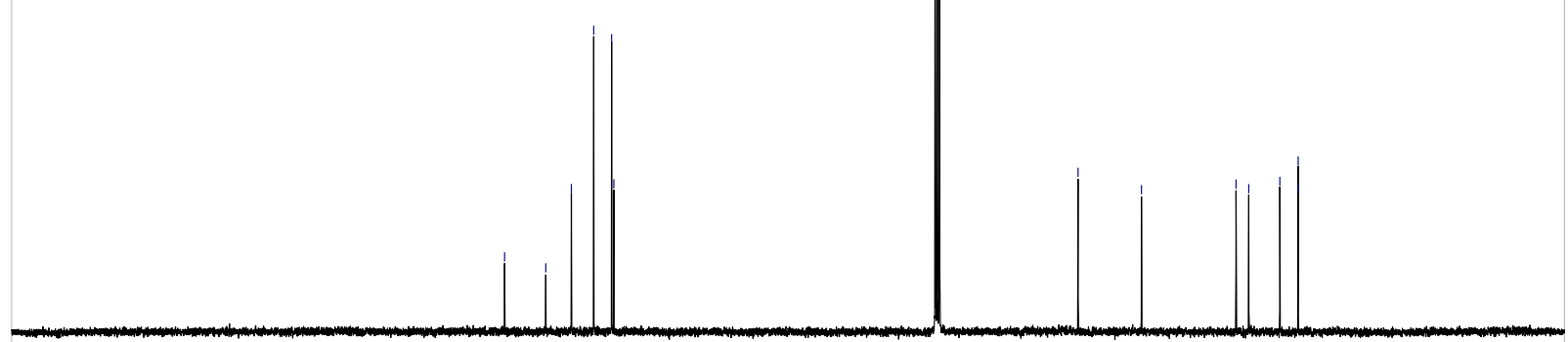

$\begin{array}{lllllllllllllllllllllll}210 & 200 & 190 & 180 & 170 & 160 & 150 & 140 & 130 & 120 & 110 & 100 & 90 & 80 & 70 & 60 & 50 & 40 & 30 & 20 & 10 & 0 & -10\end{array}$ 
<smiles>CC1=CCCCC1N(C)CCBr</smiles>

$\mathrm{CDCl}_{3}, 400 \mathrm{MHz}$

$1 \mathrm{~s}$
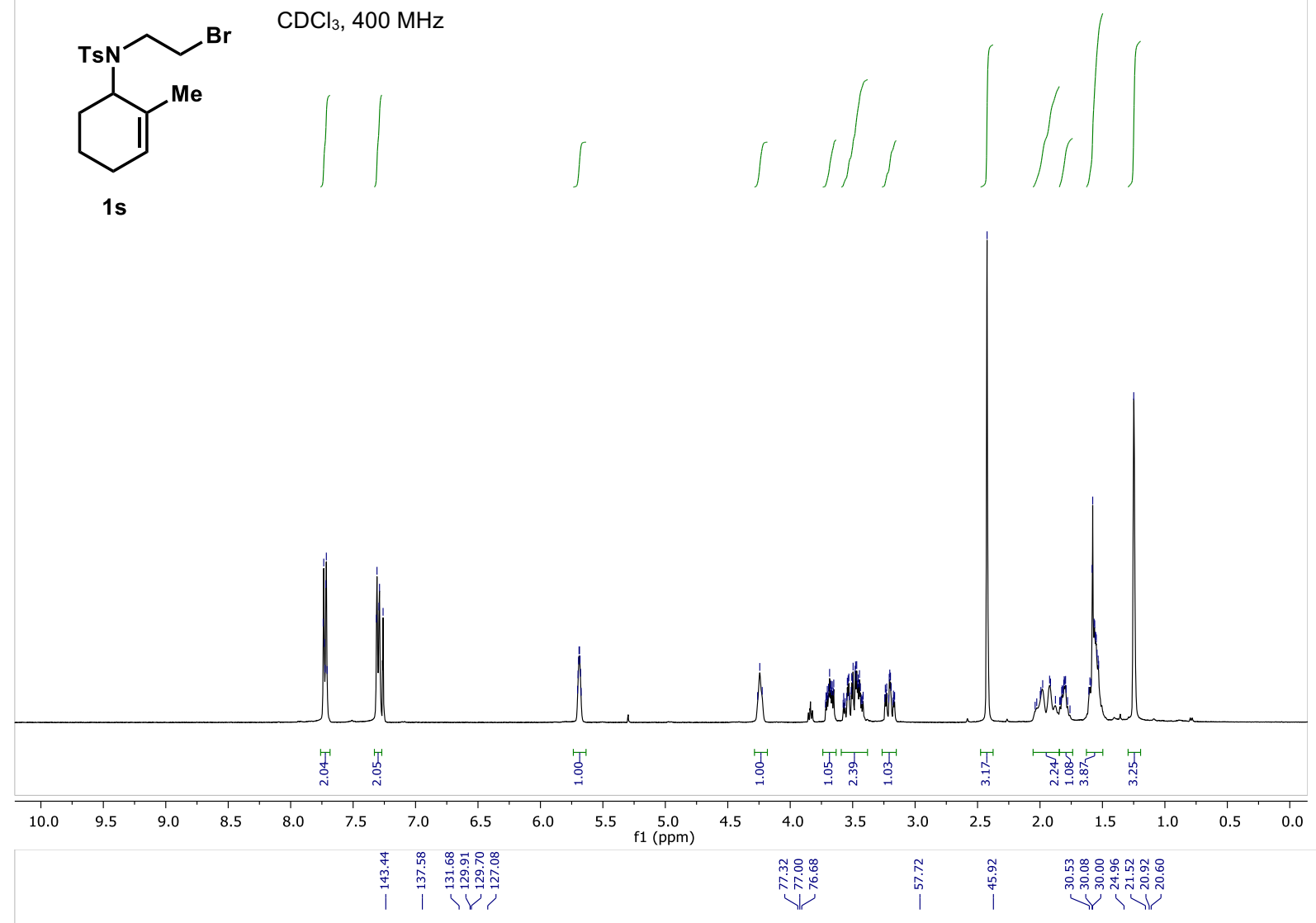

$\mathrm{CDCl}_{3}, 101 \mathrm{MHz}$

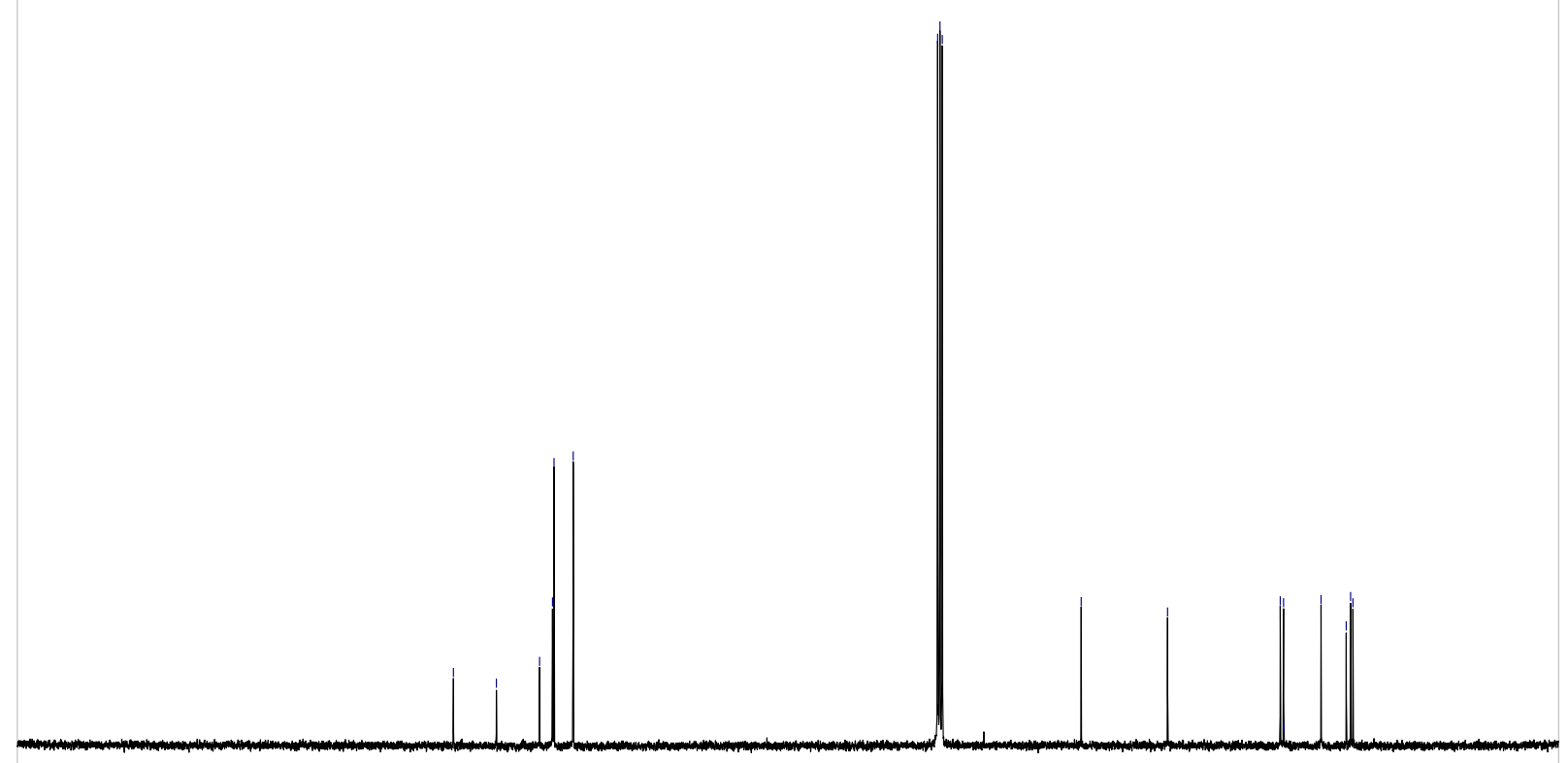

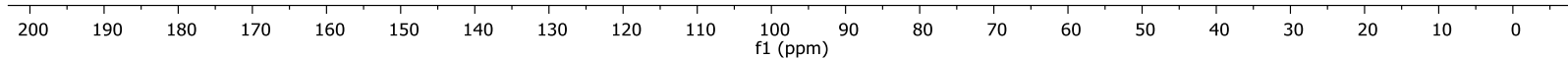




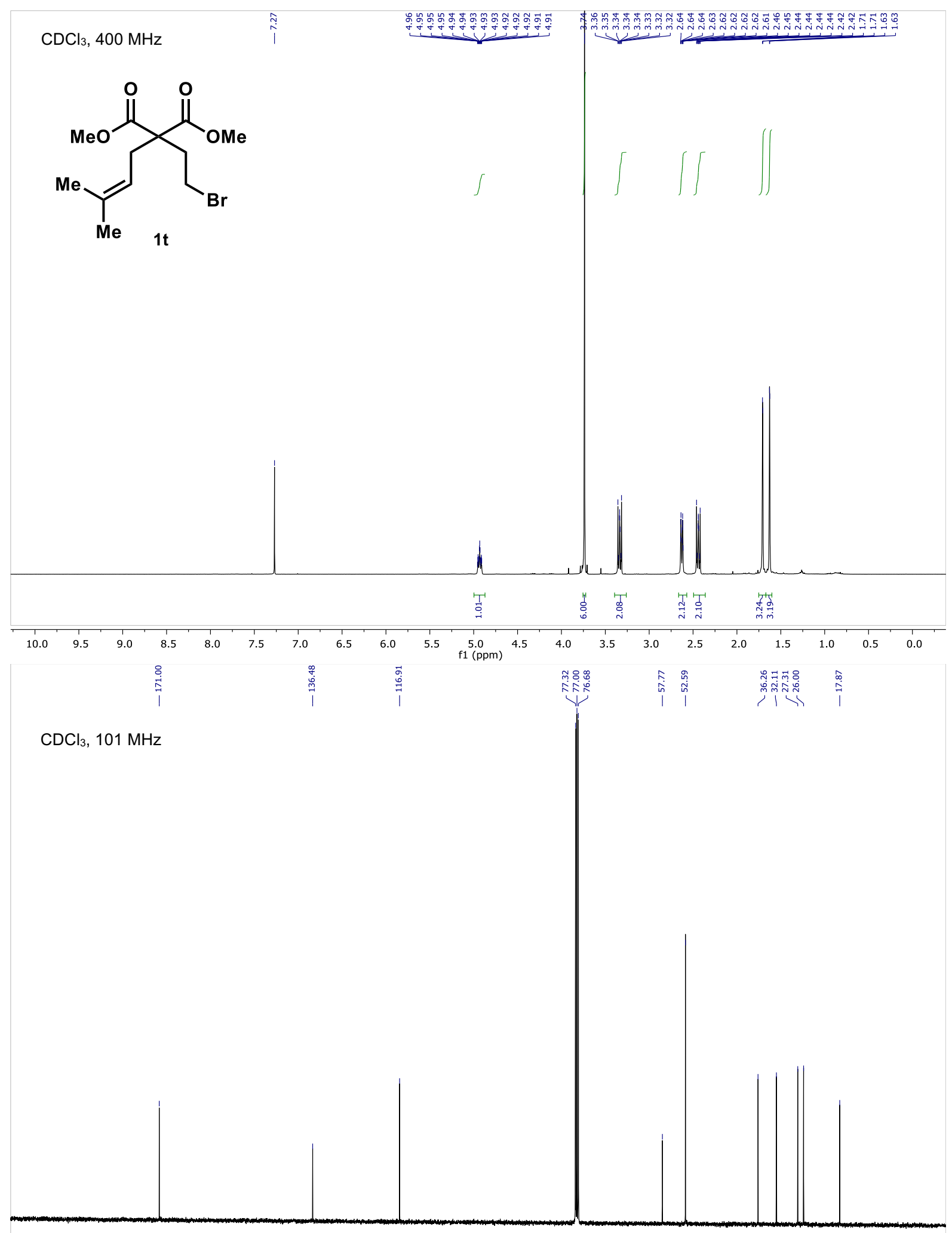

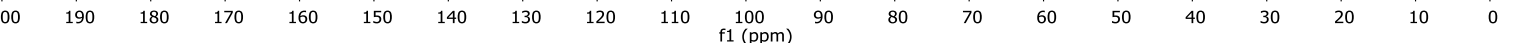


$\mathrm{CDCl}_{3}, 400 \mathrm{MHz}$<smiles>COC(=O)C1(C(=O)OC)CCC(CBr)C1</smiles>

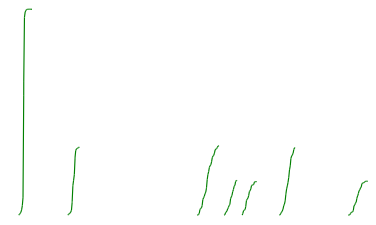

2a
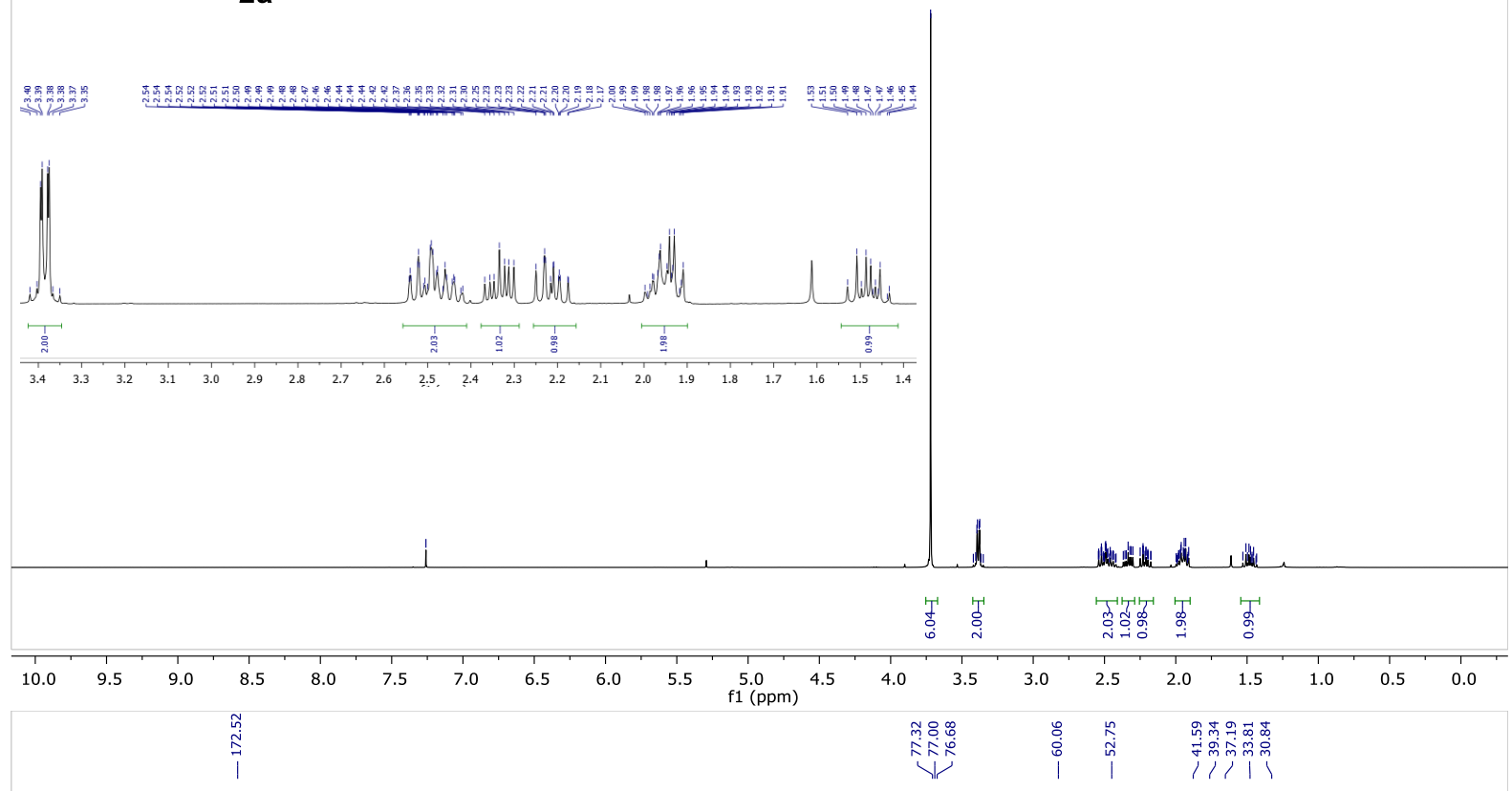

$\mathrm{CDCl}_{3}, 101 \mathrm{MHz}$

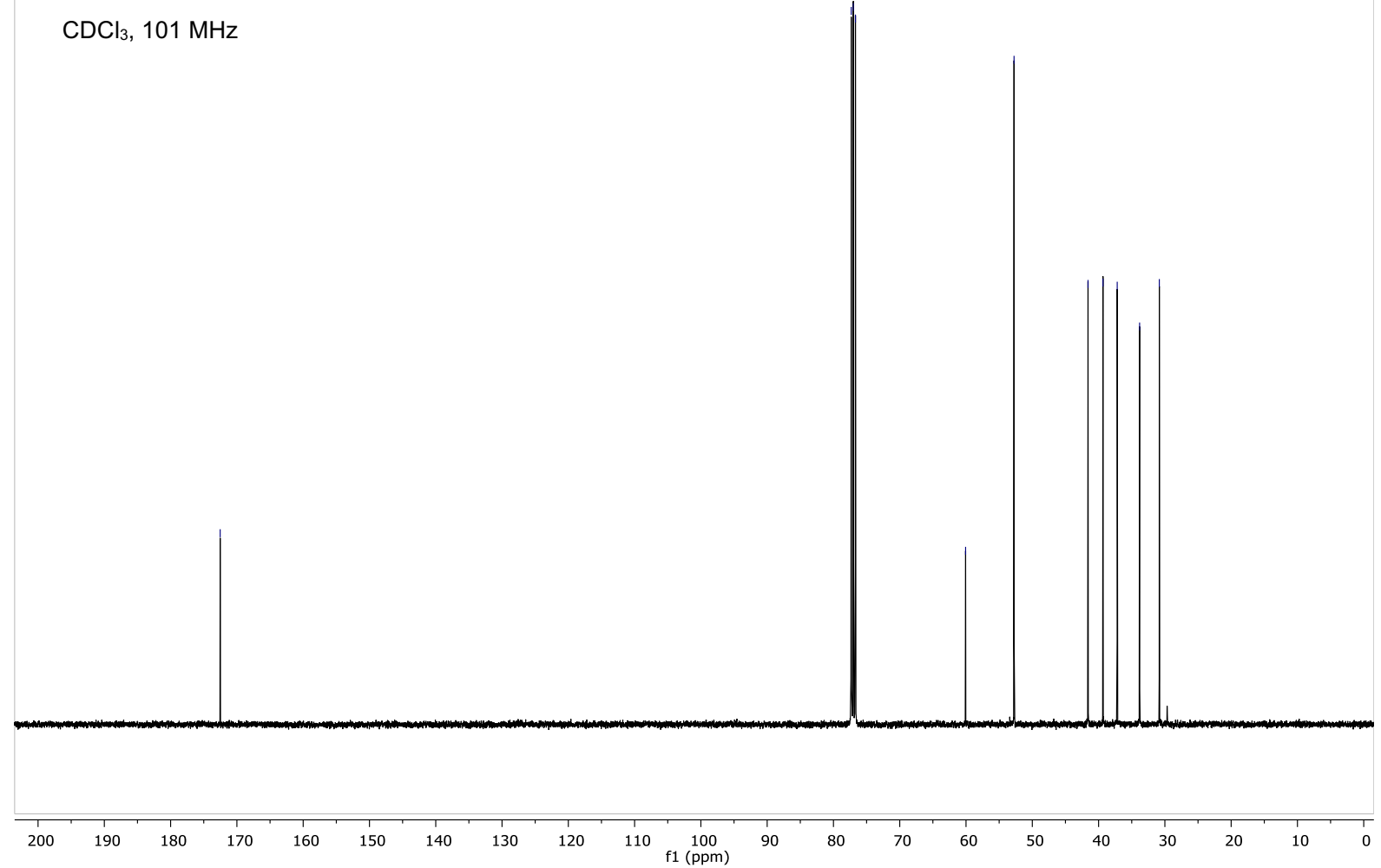



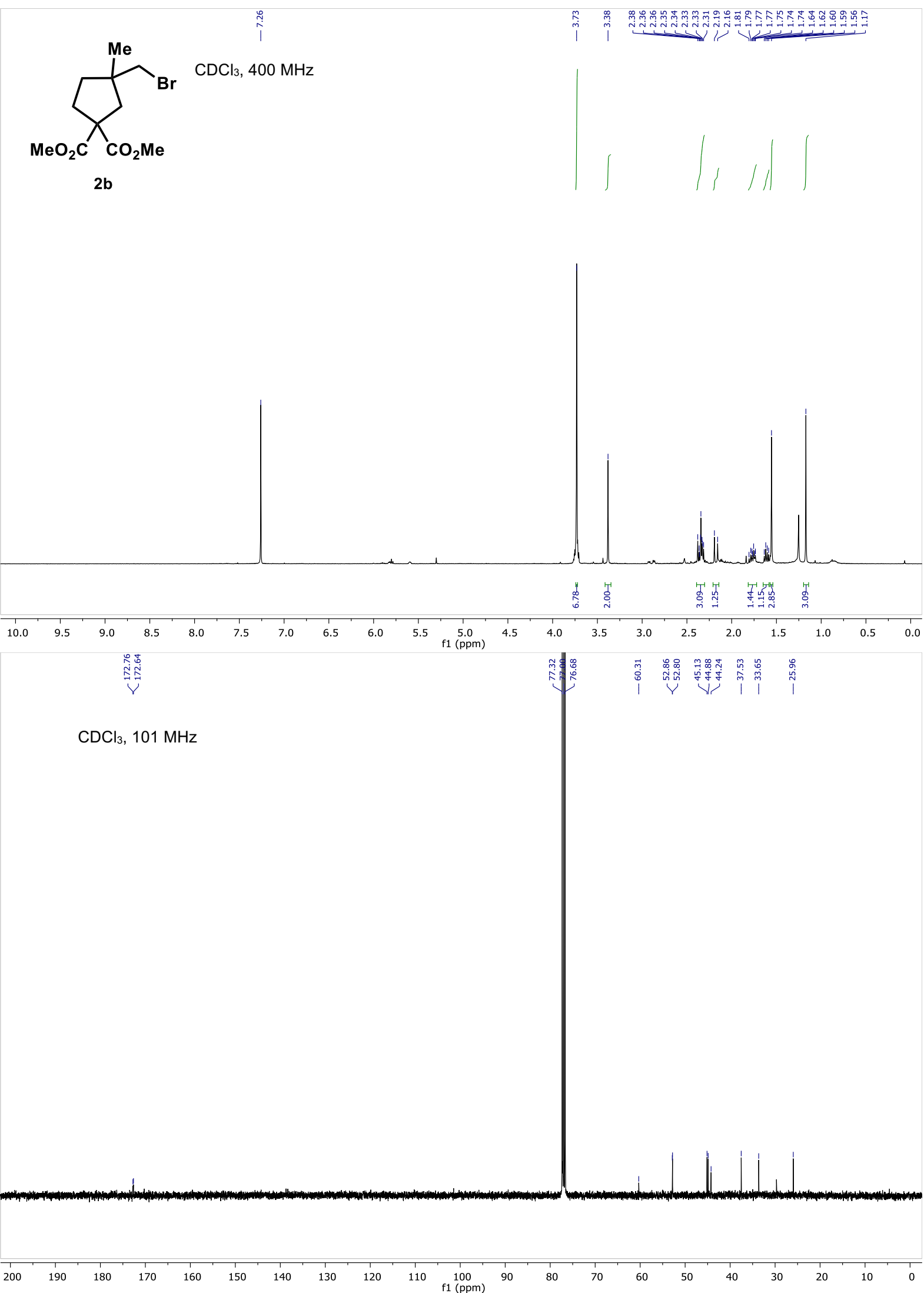

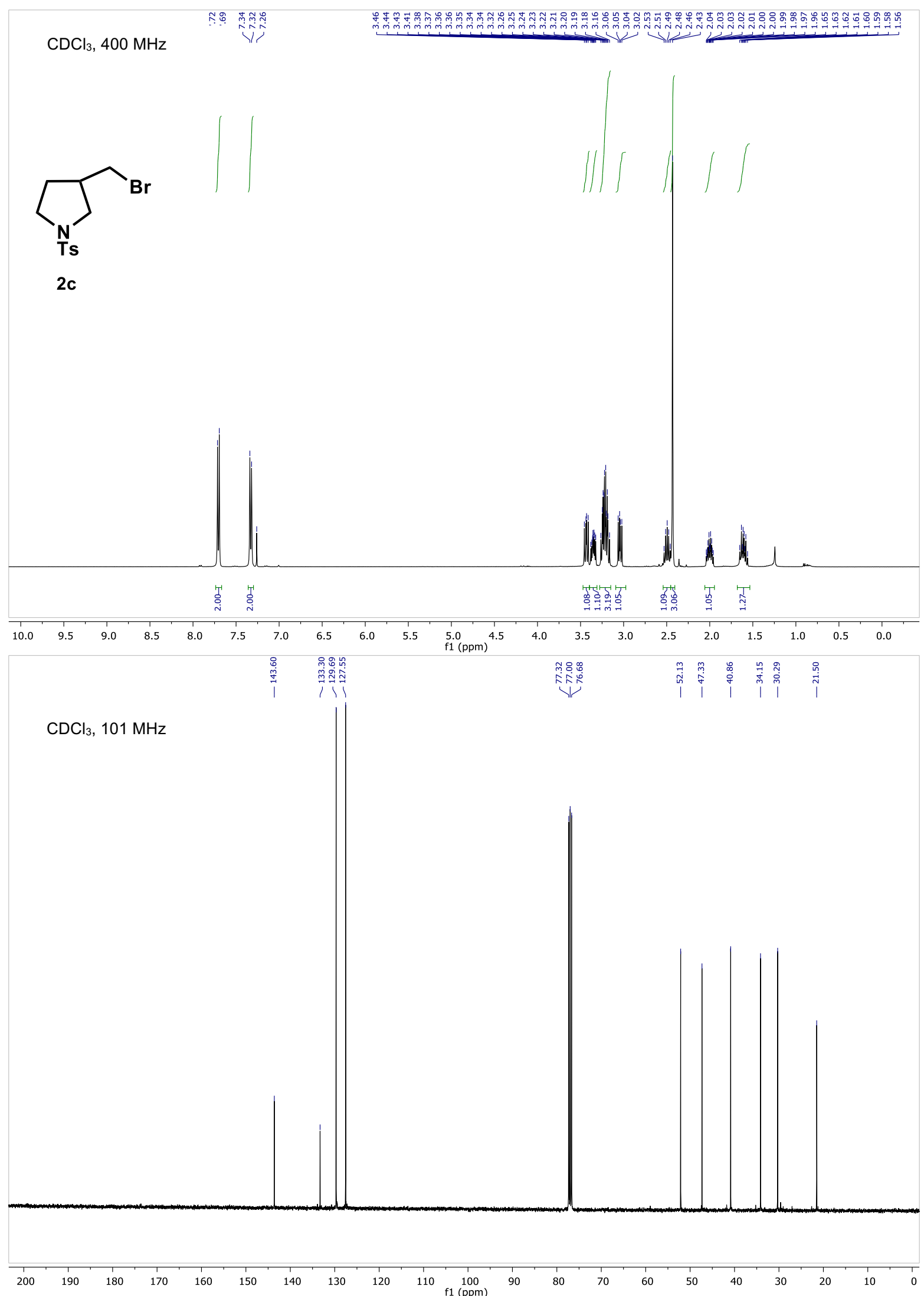

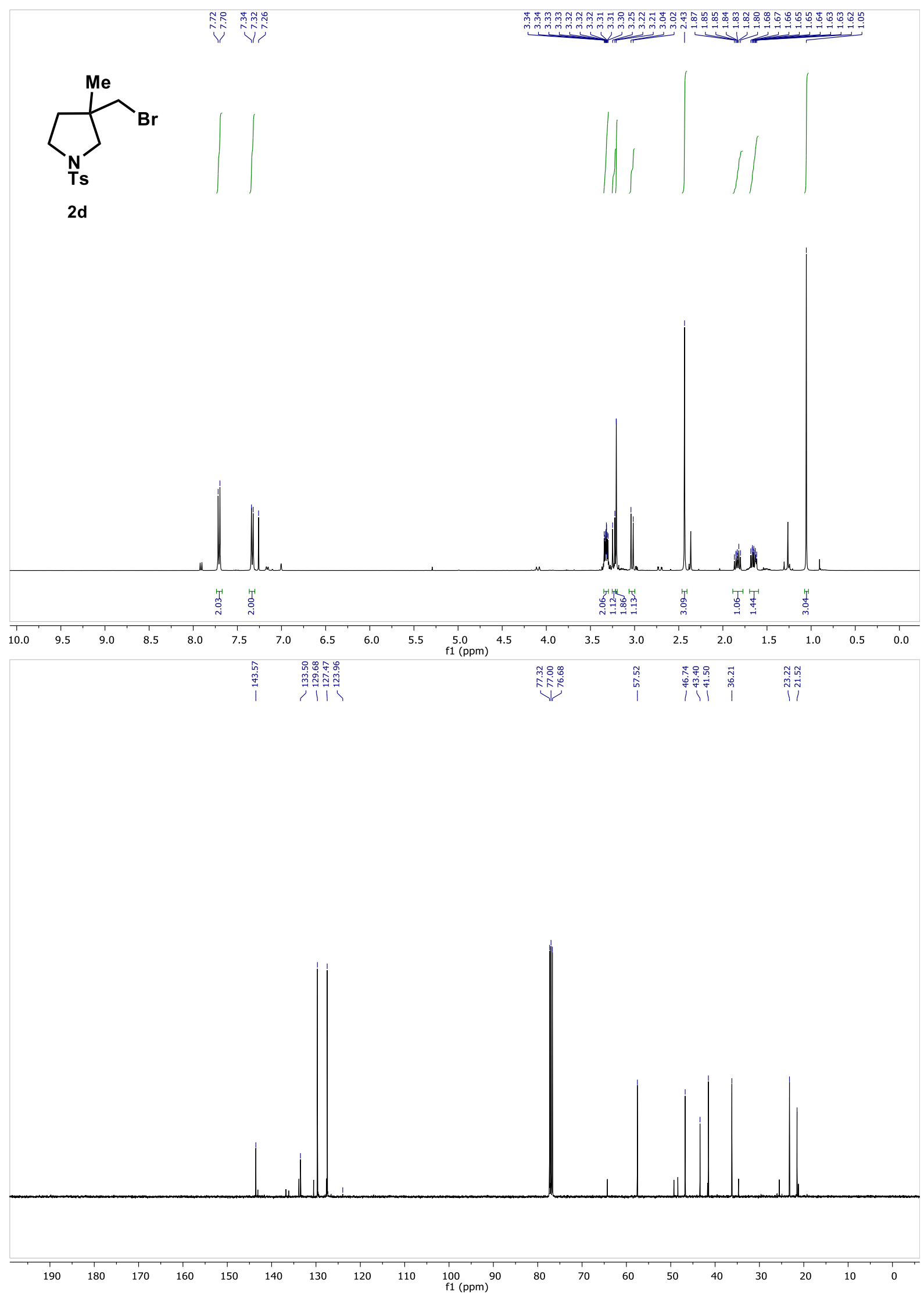

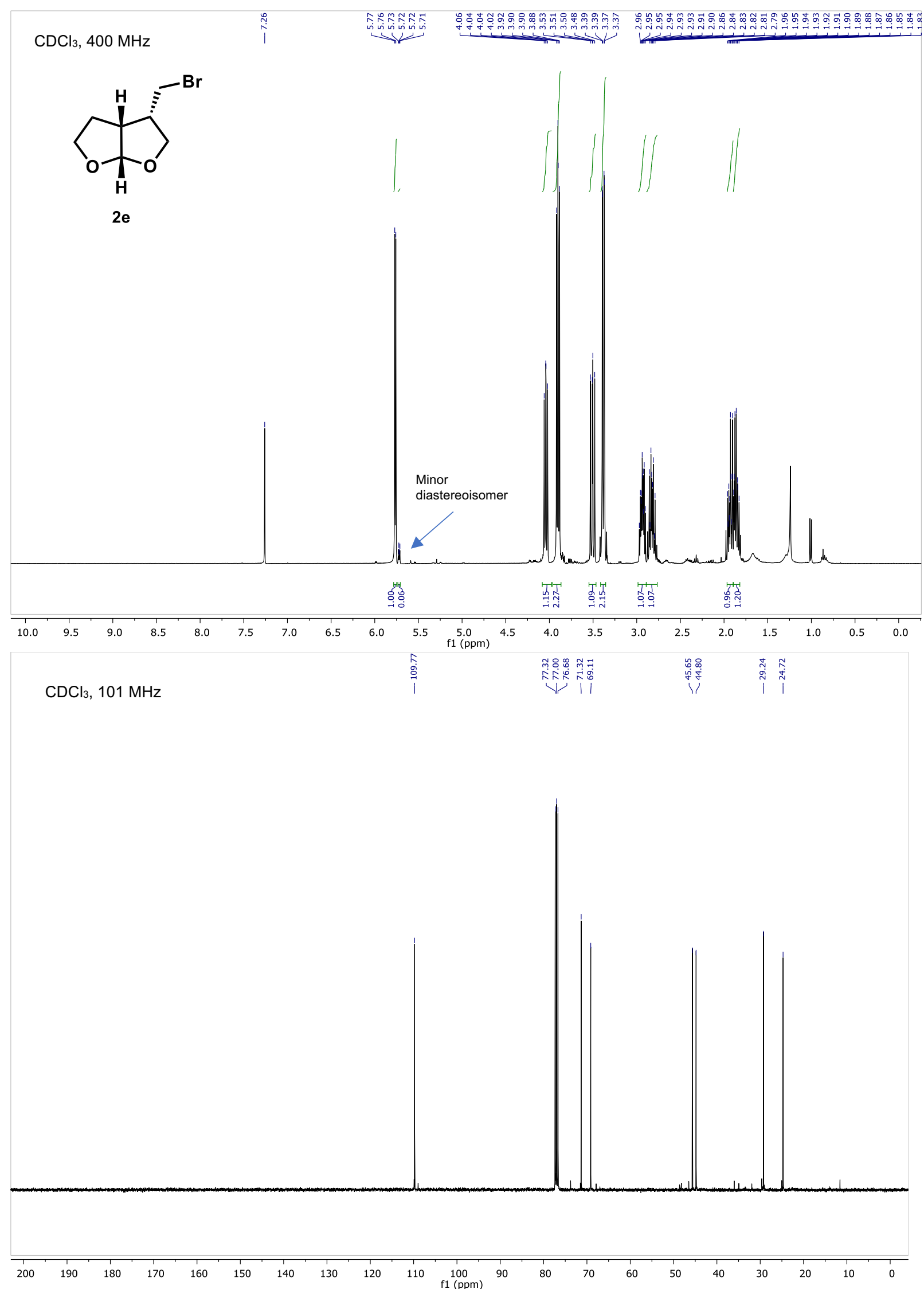


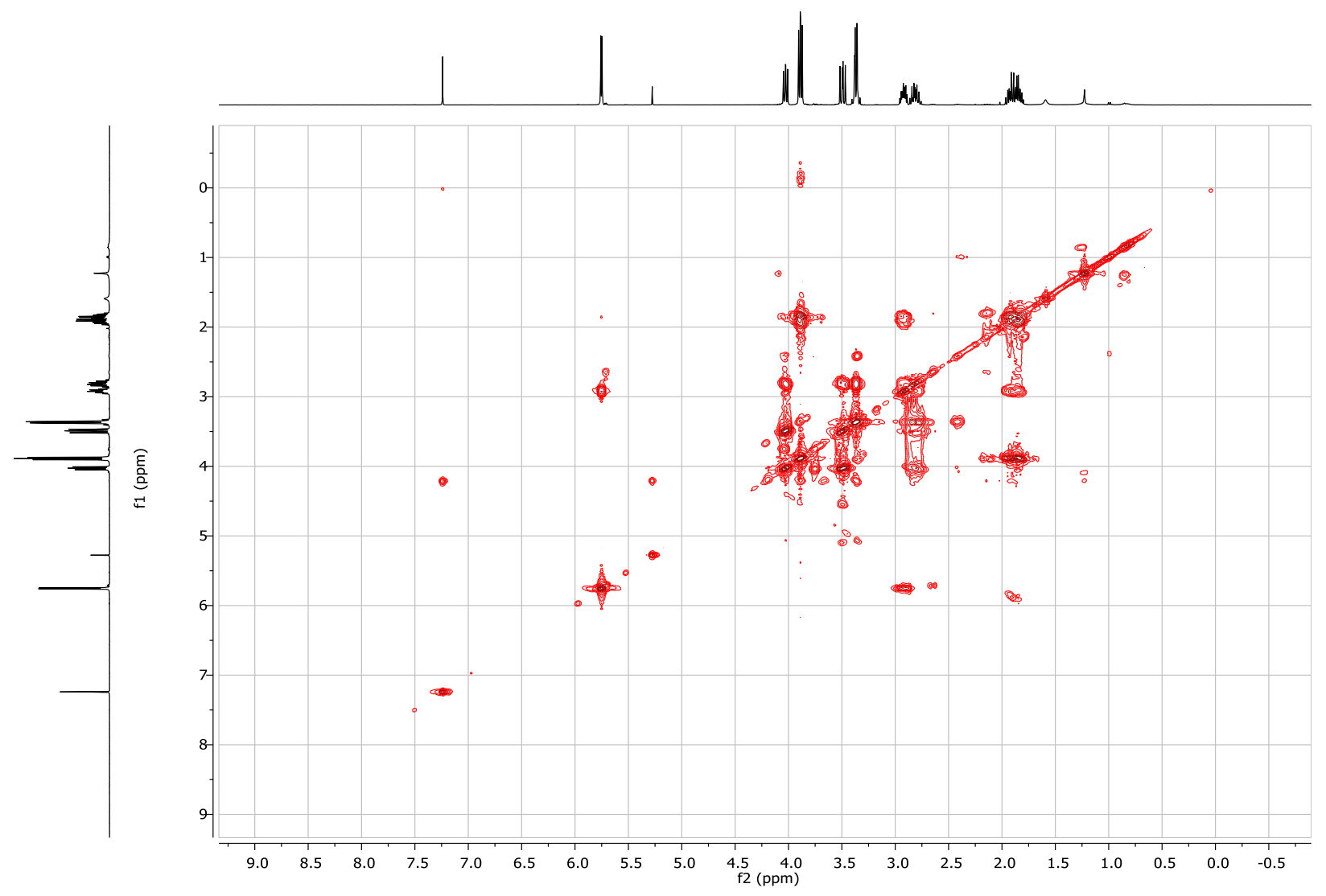



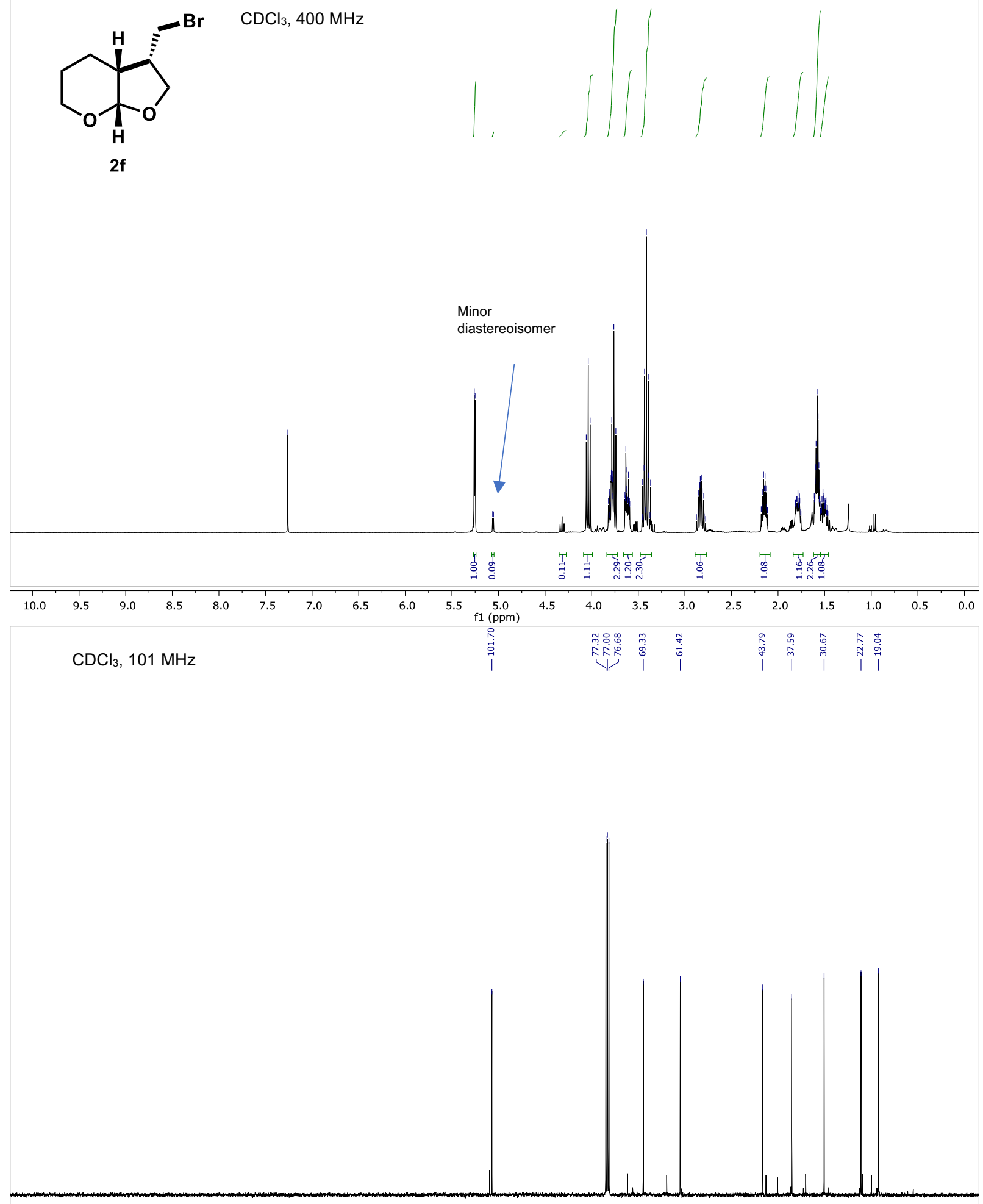

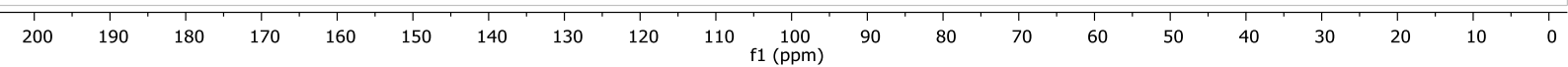




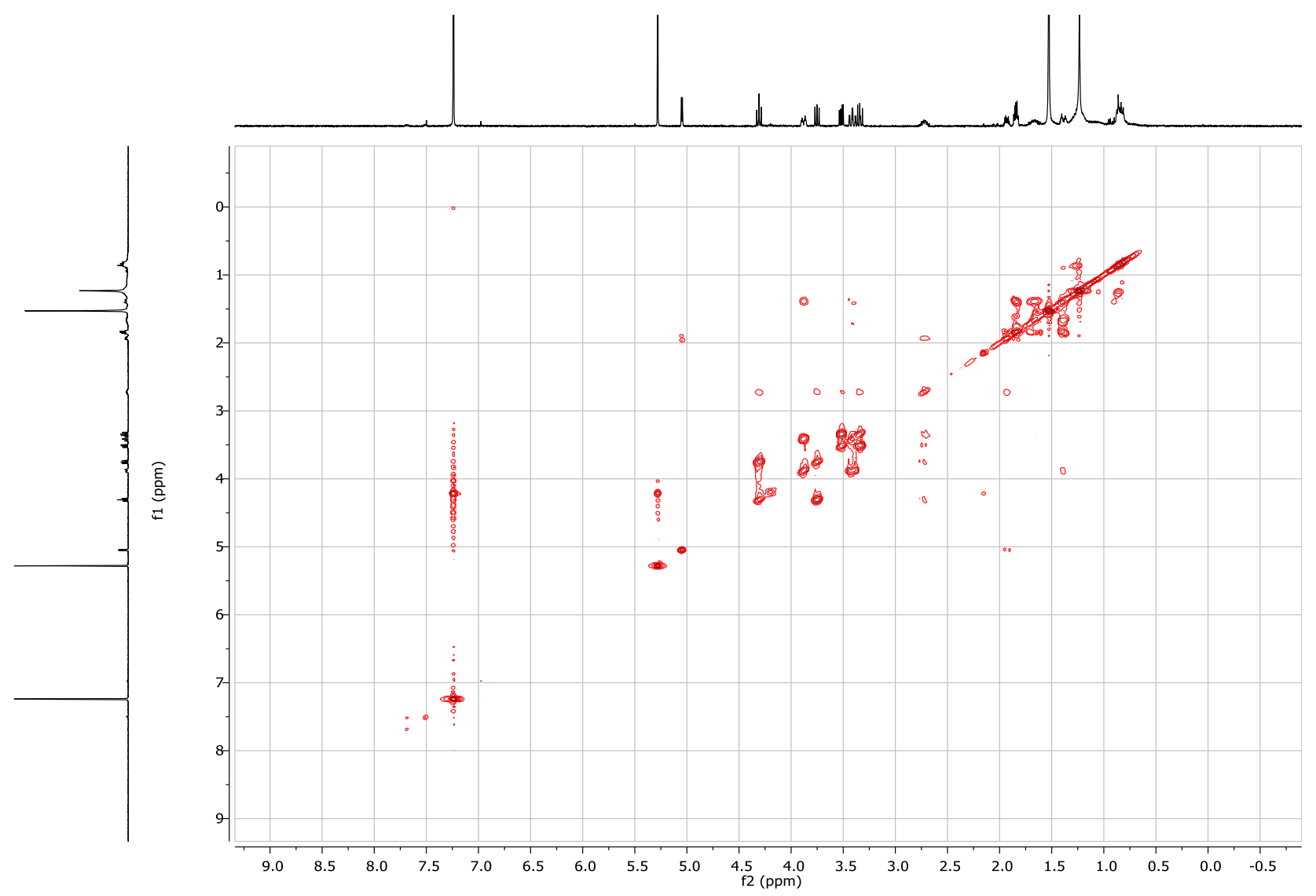




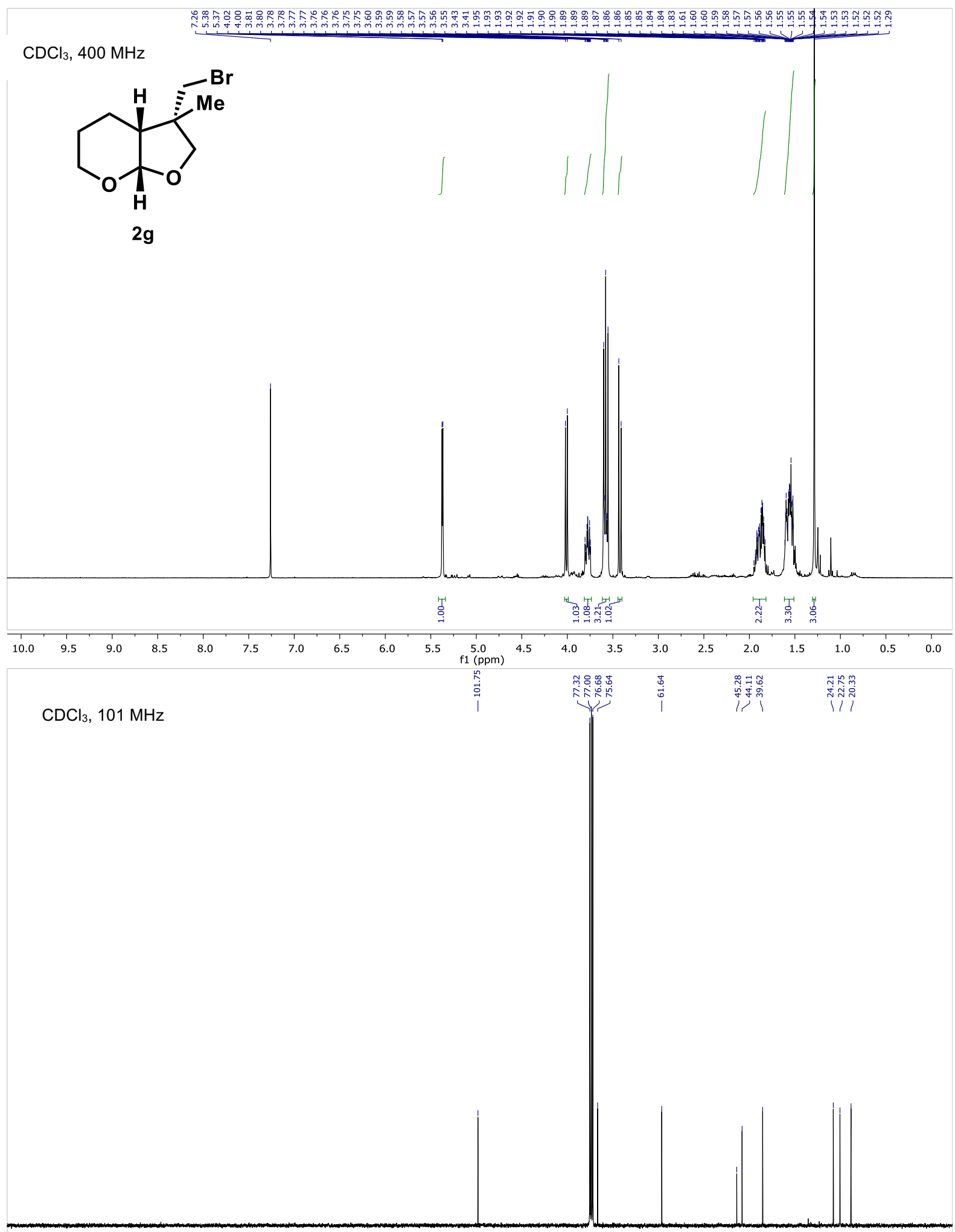

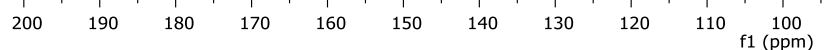




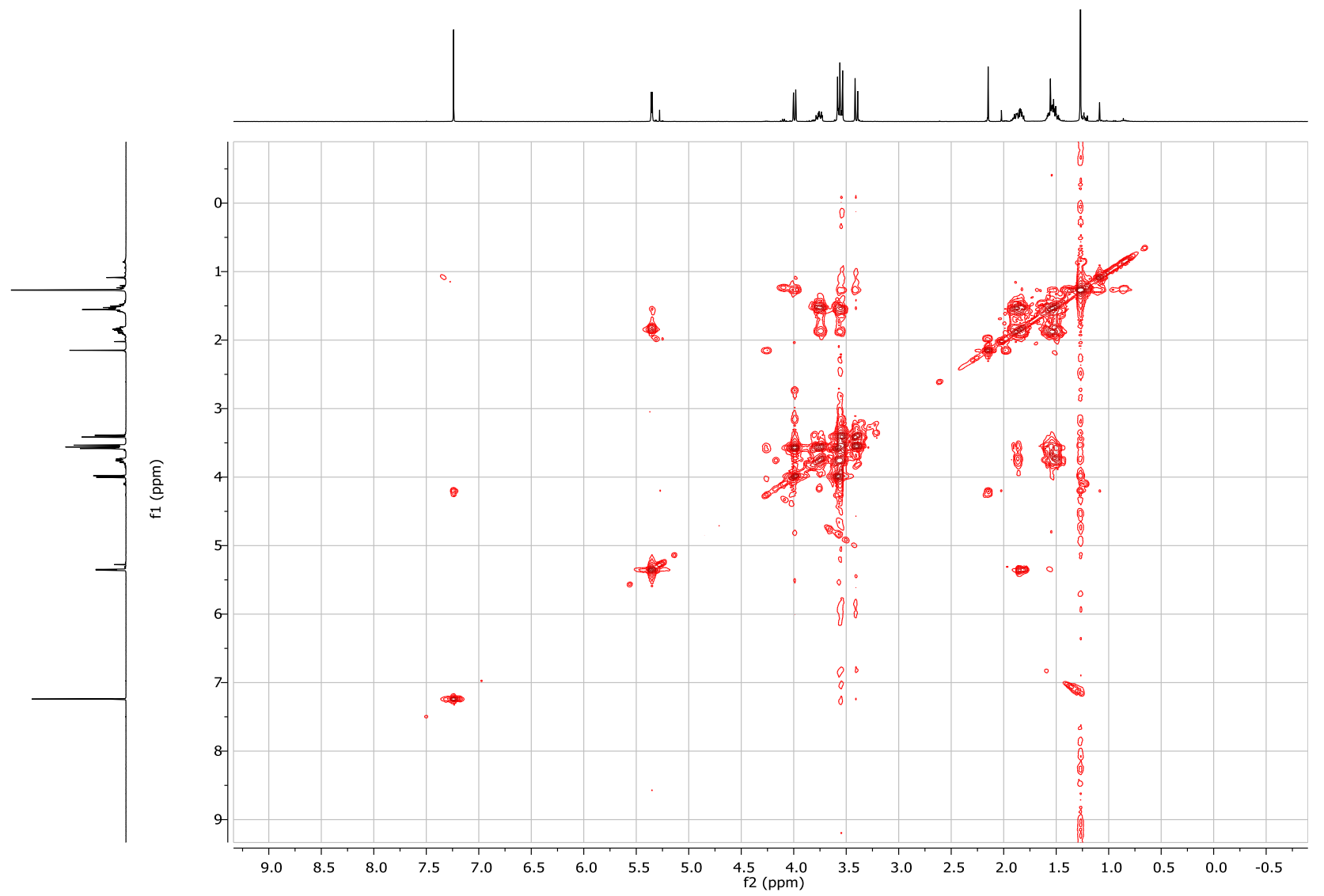




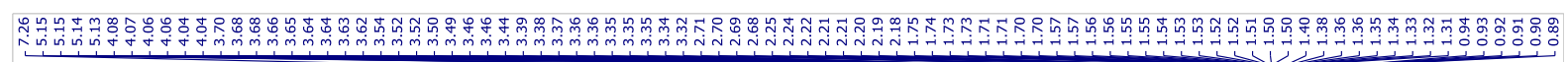
$\mathrm{CDCl}_{3}, 400 \mathrm{MHz}$<smiles>CCCCO[C@H]1C[C@@H](CBr)CO1</smiles>

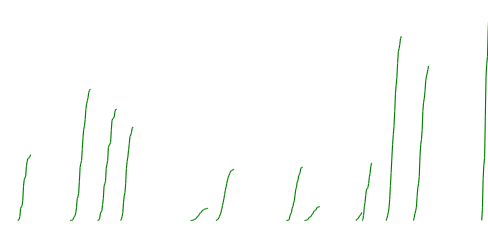

$2 h$
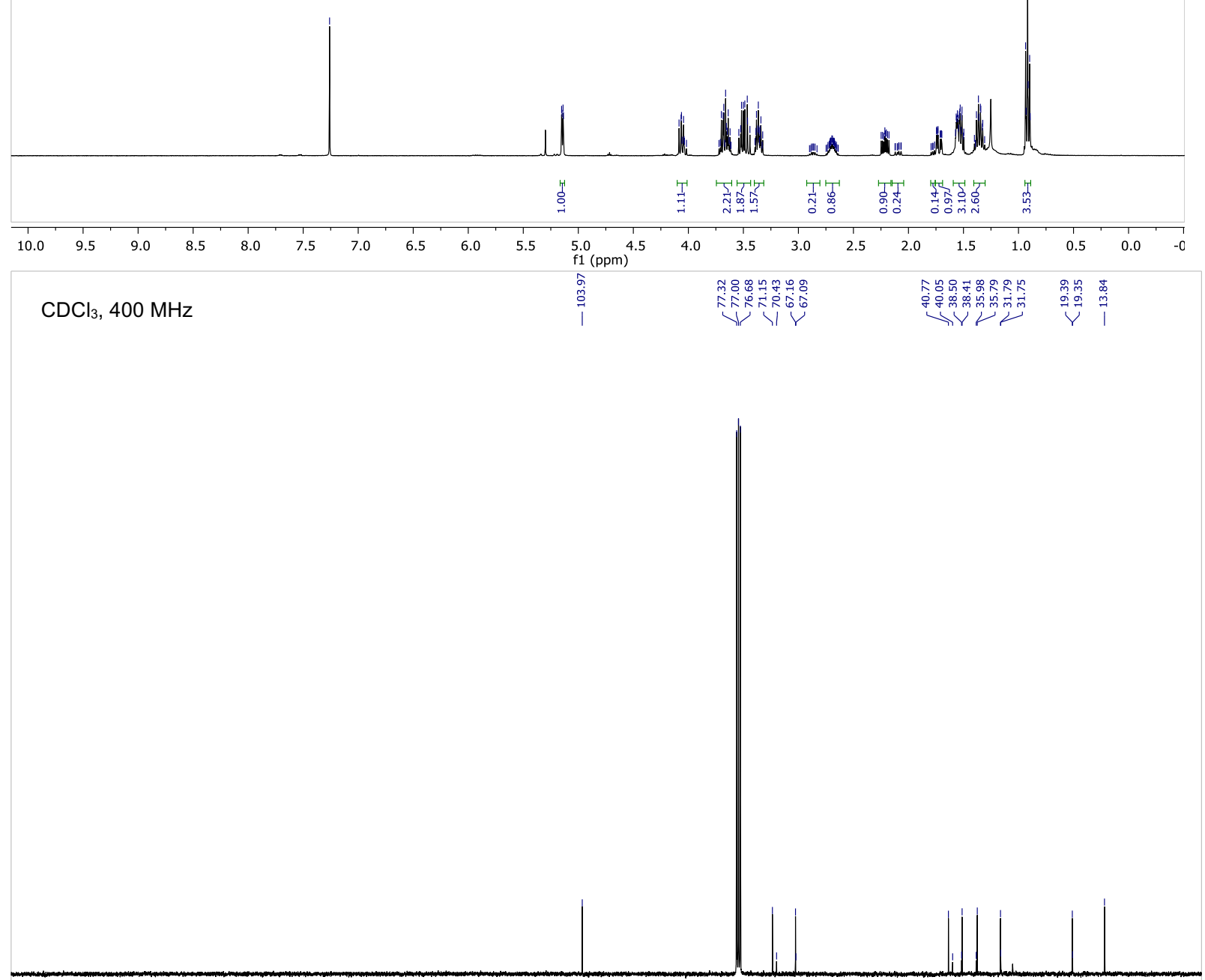

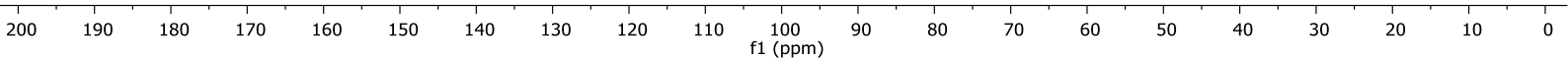


<smiles>CCCCO[C@H]1CC(C)(CBr)CO1</smiles>

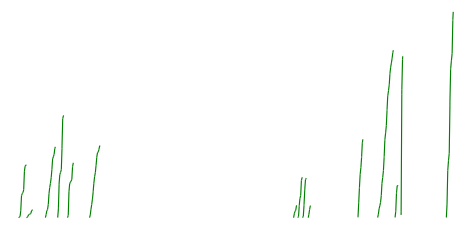

2i
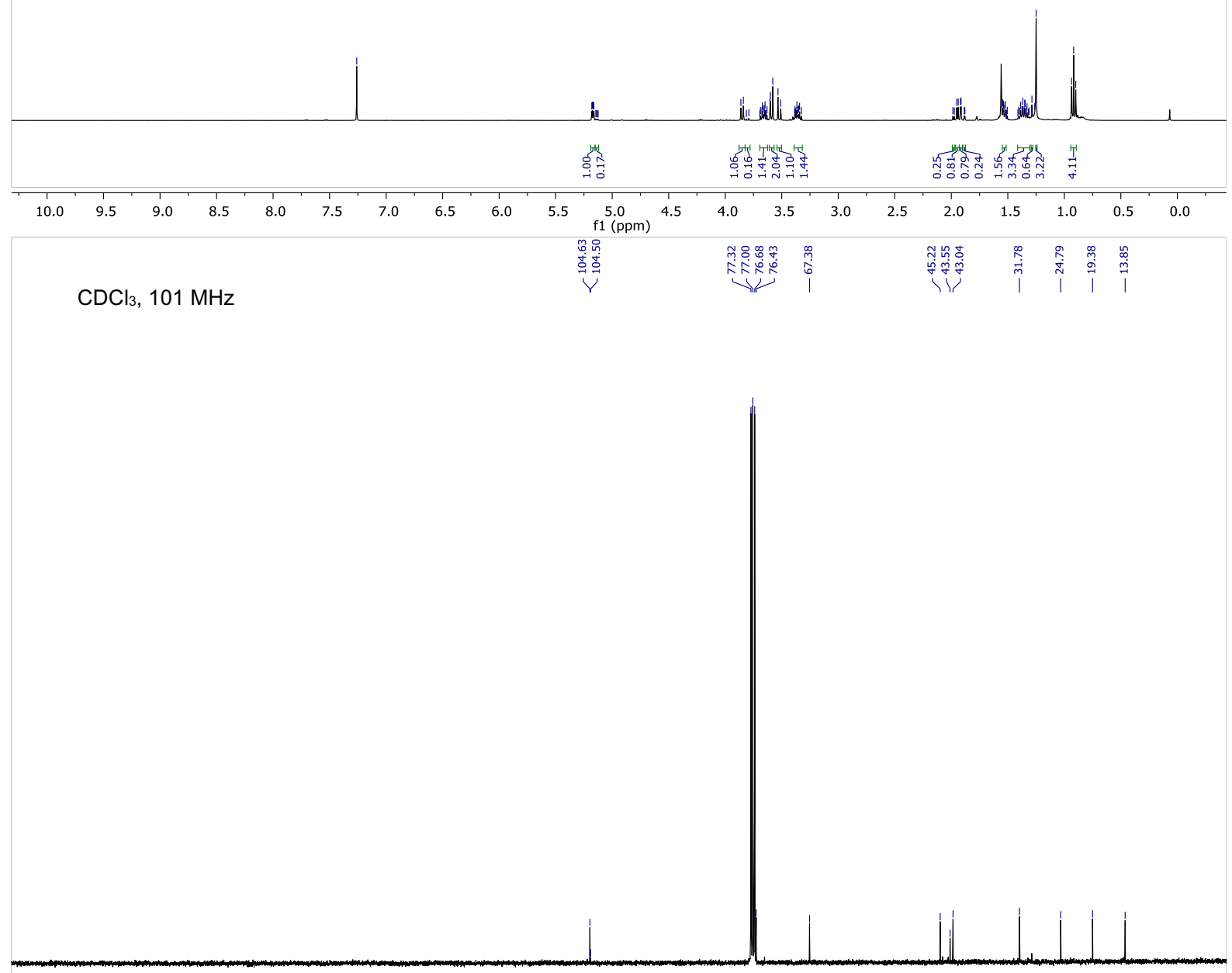

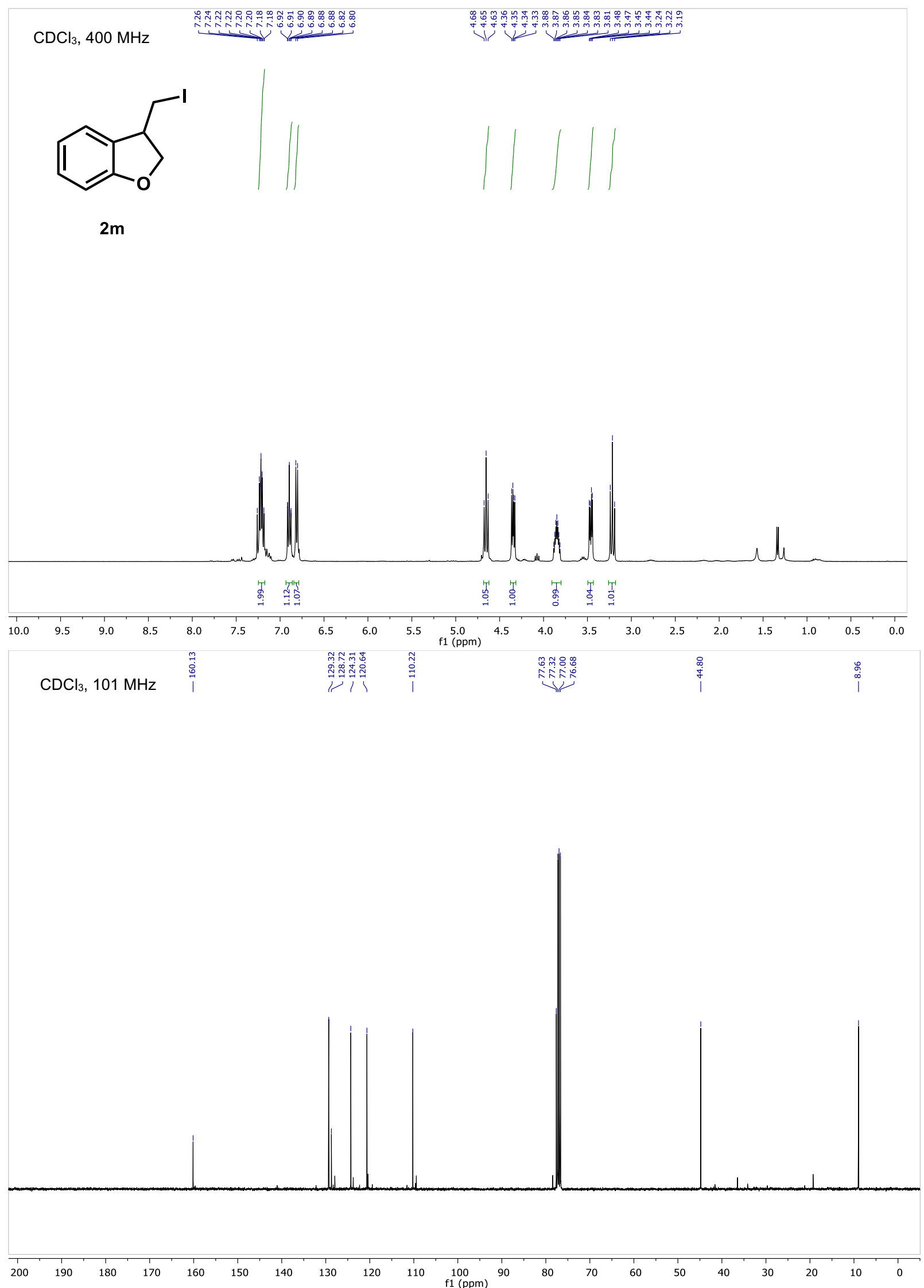

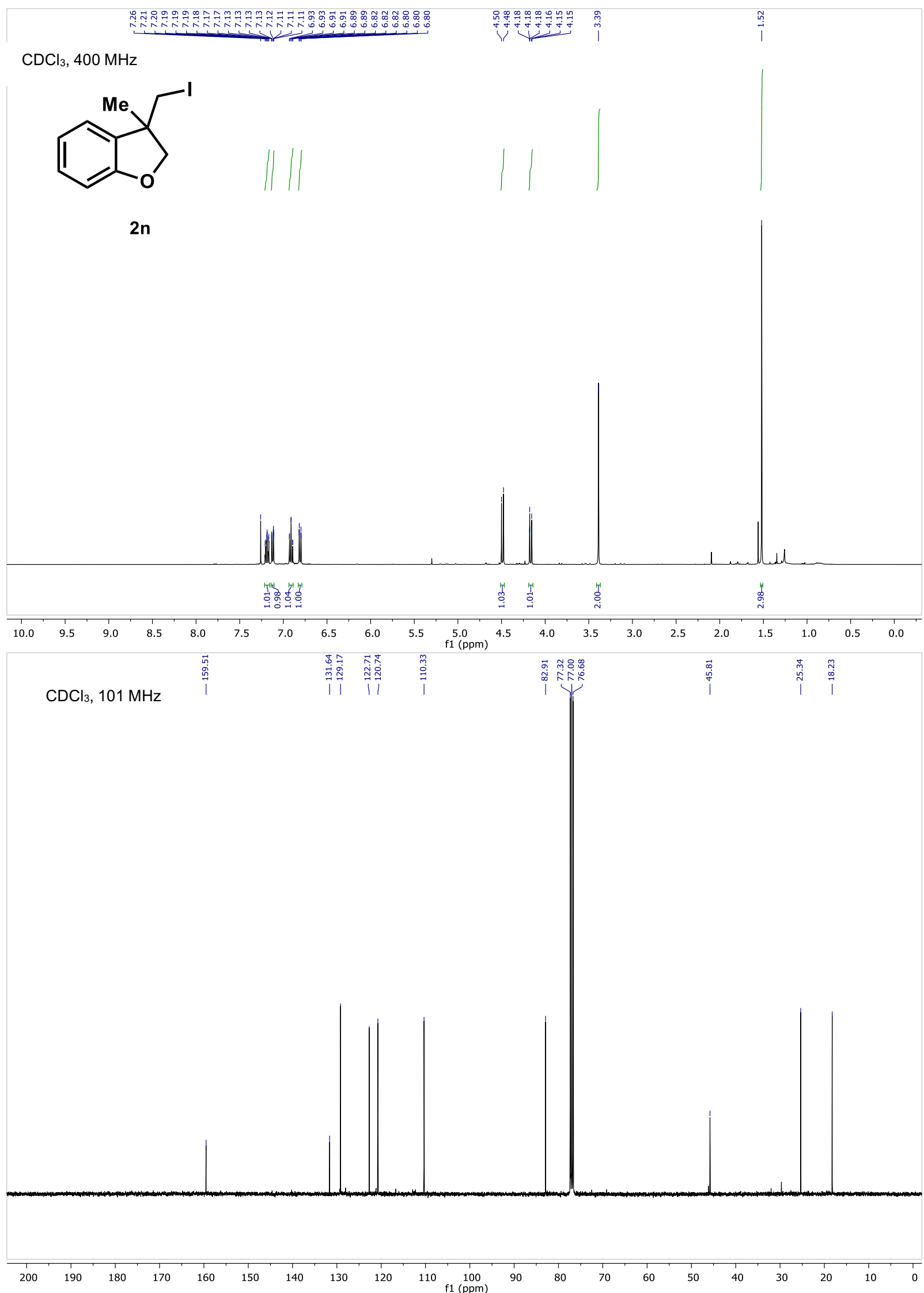

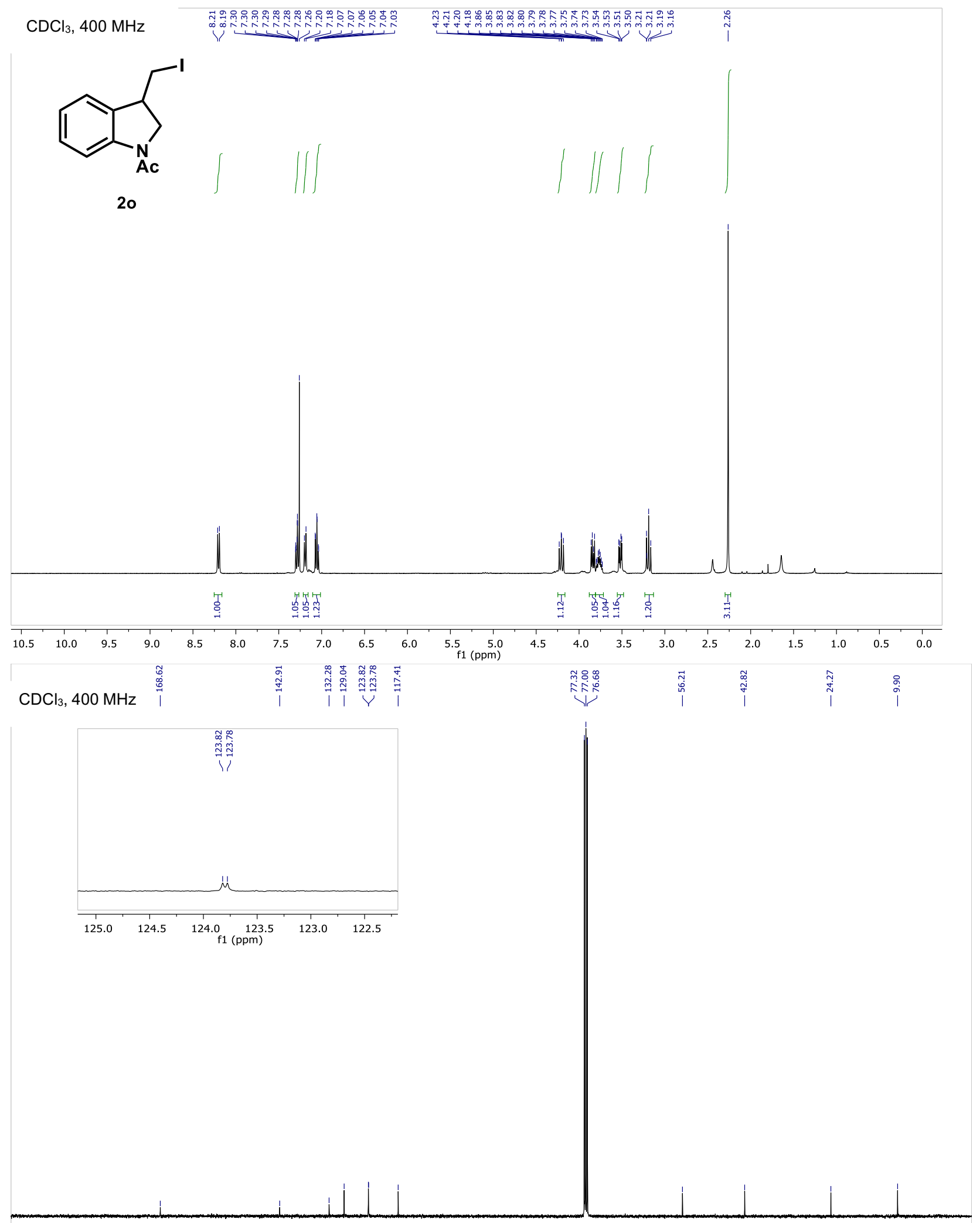

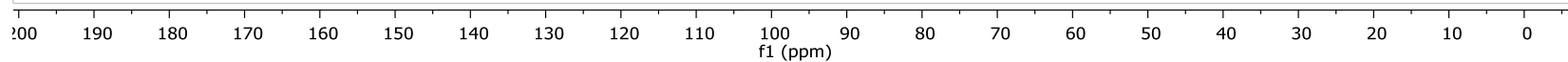


$\mathrm{CDCl}_{3}, 400 \mathrm{MHz}$

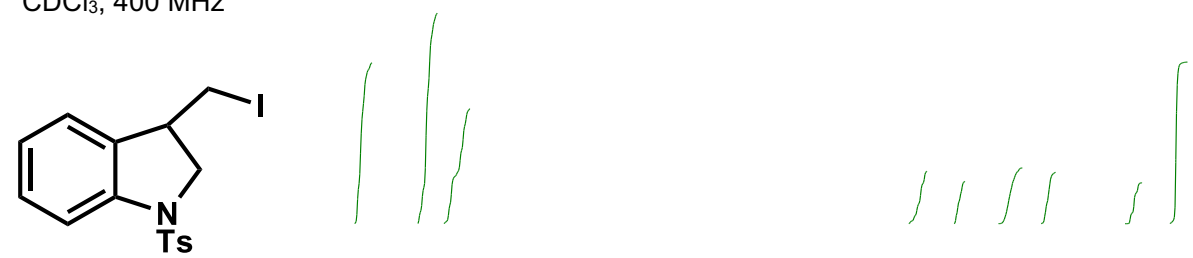

$2 p$
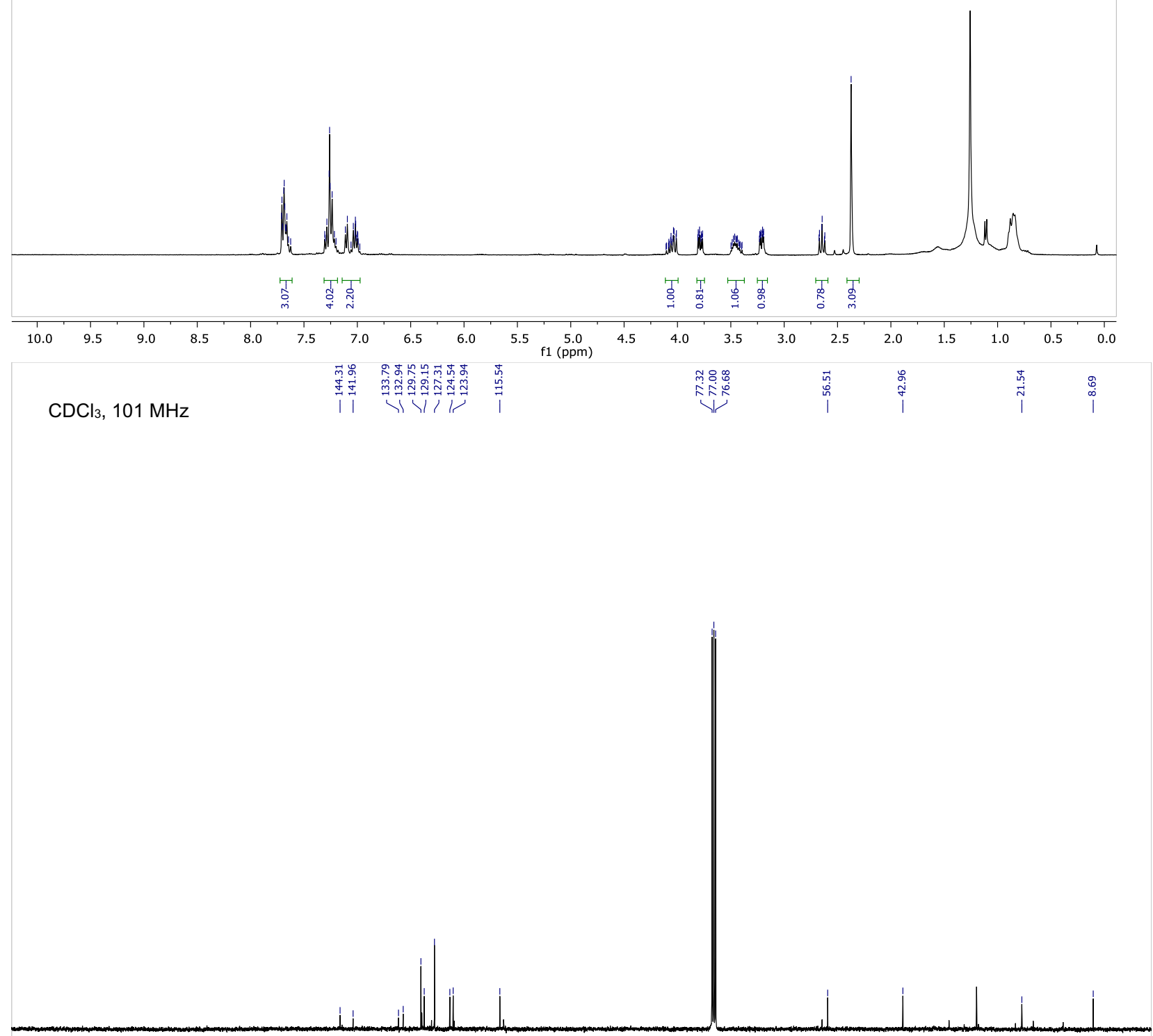

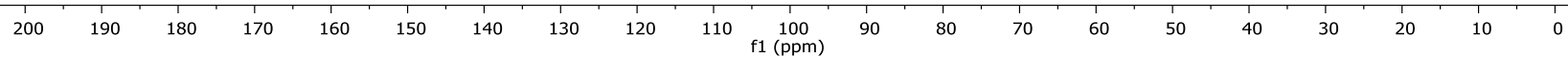



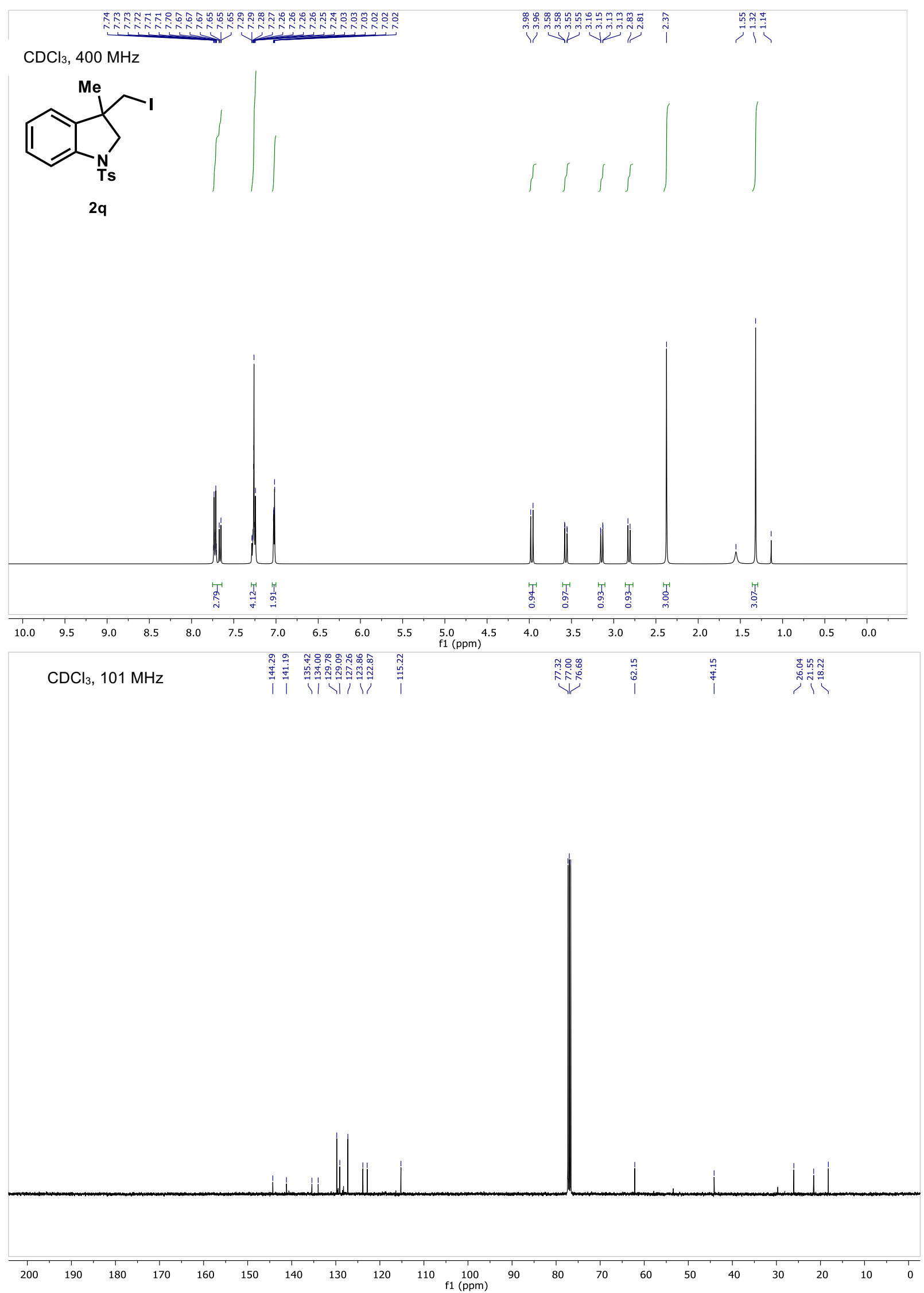

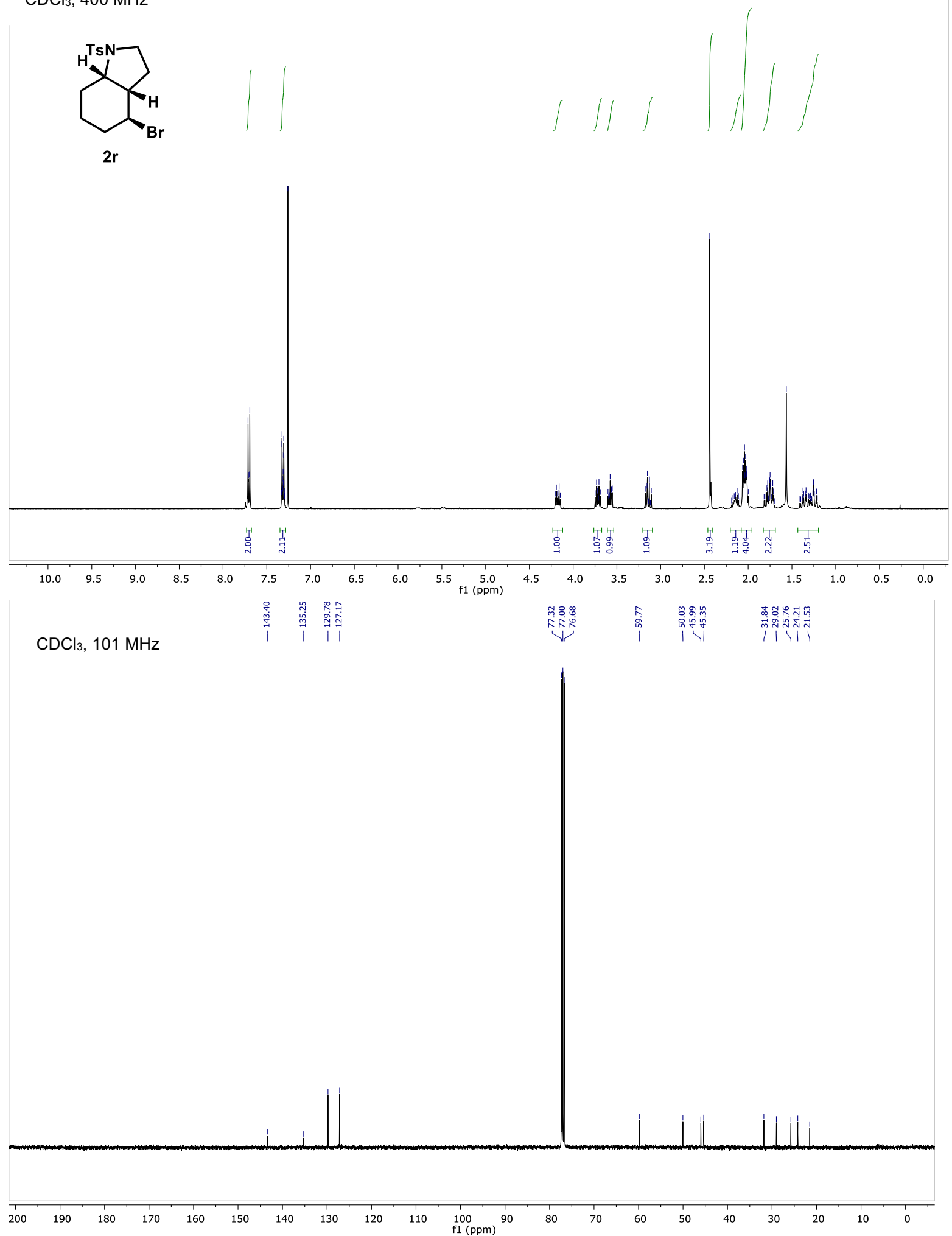


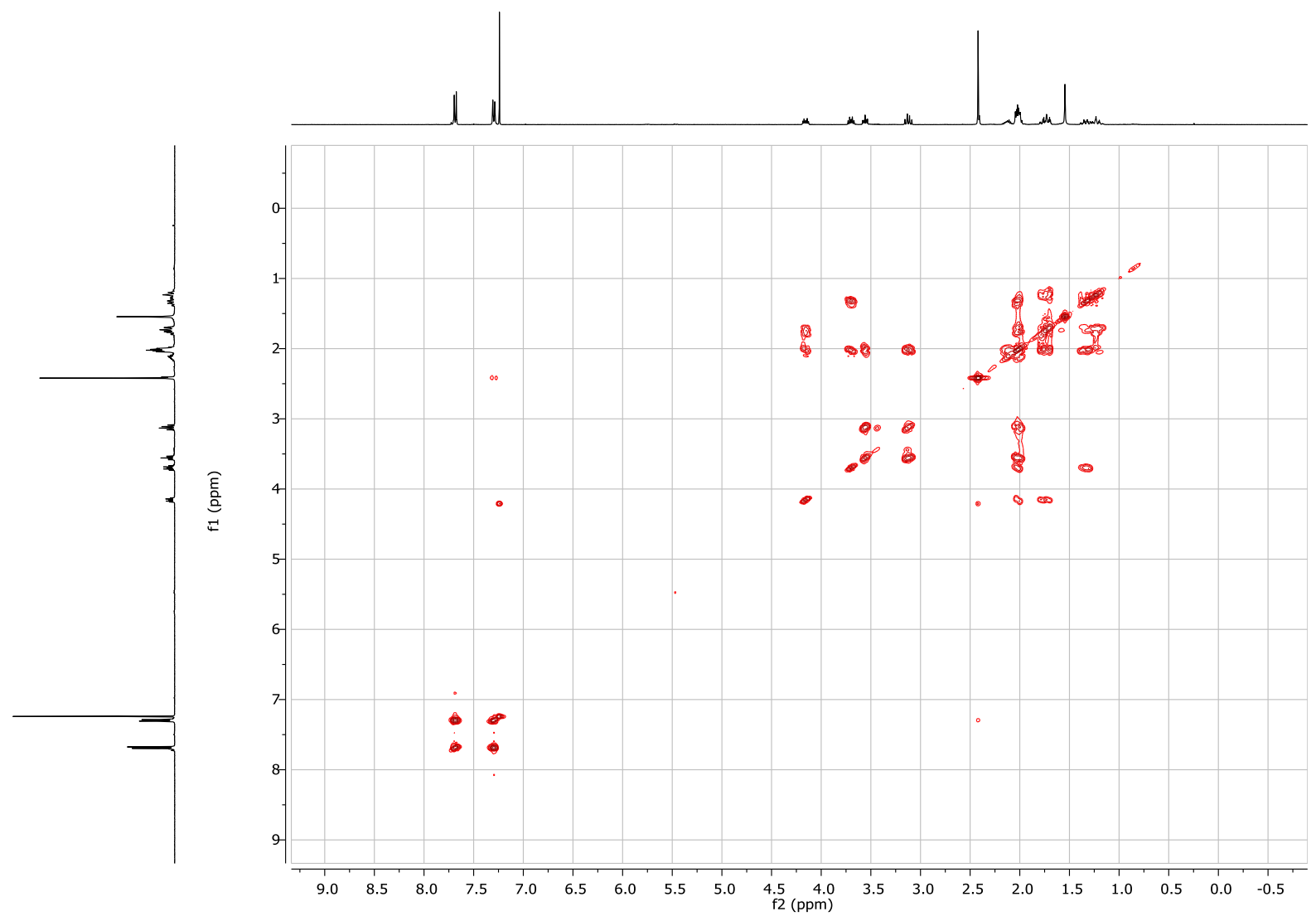



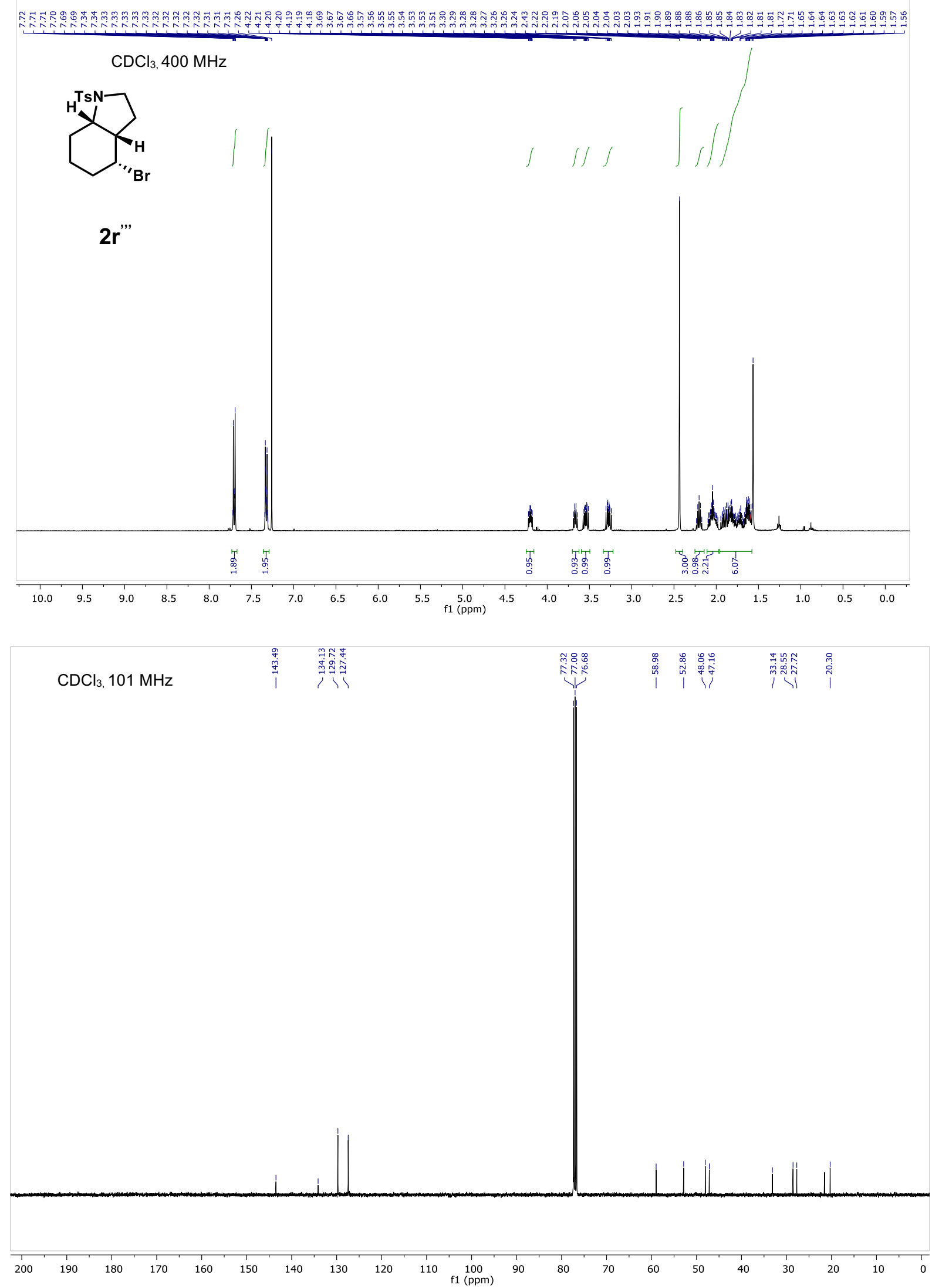


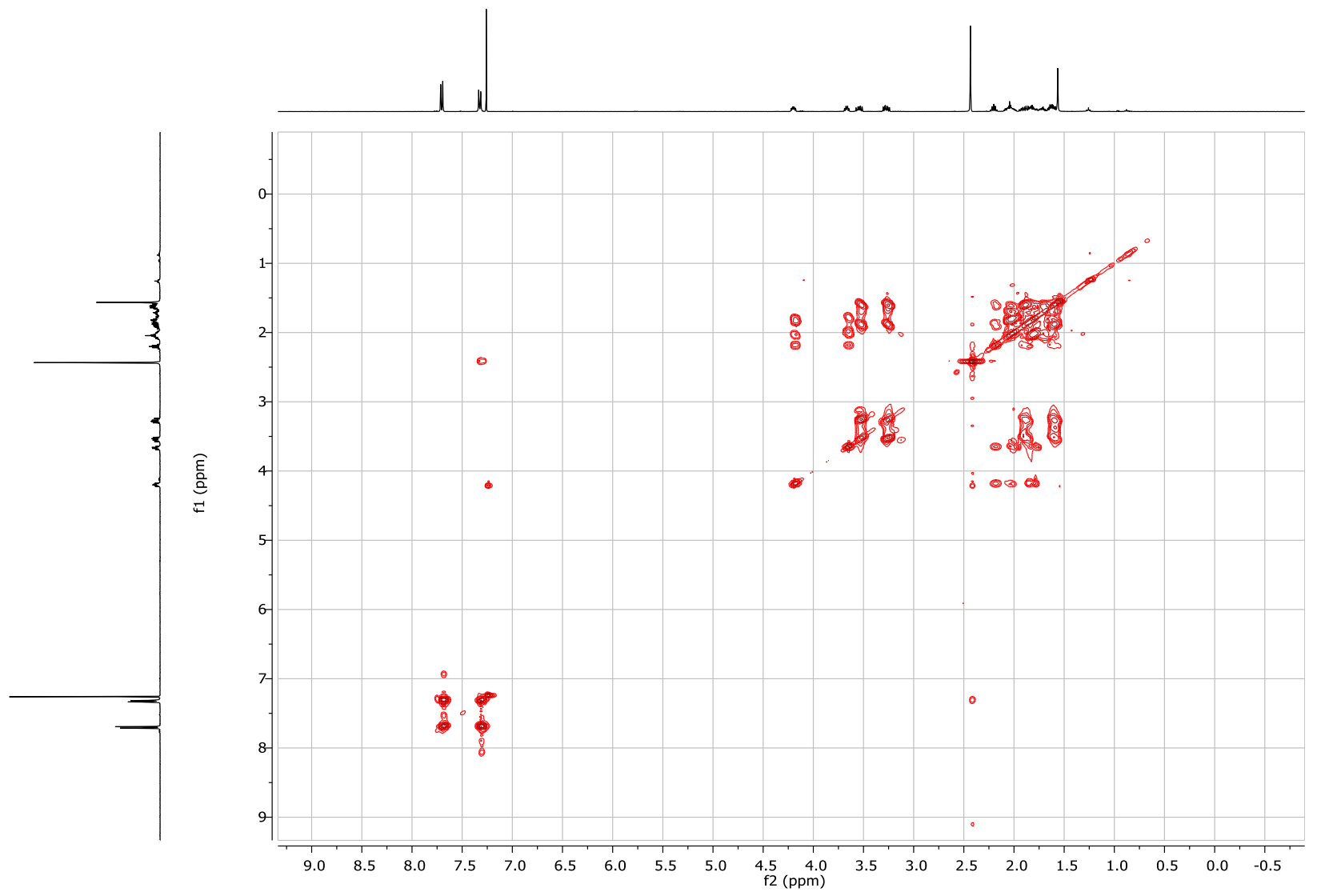



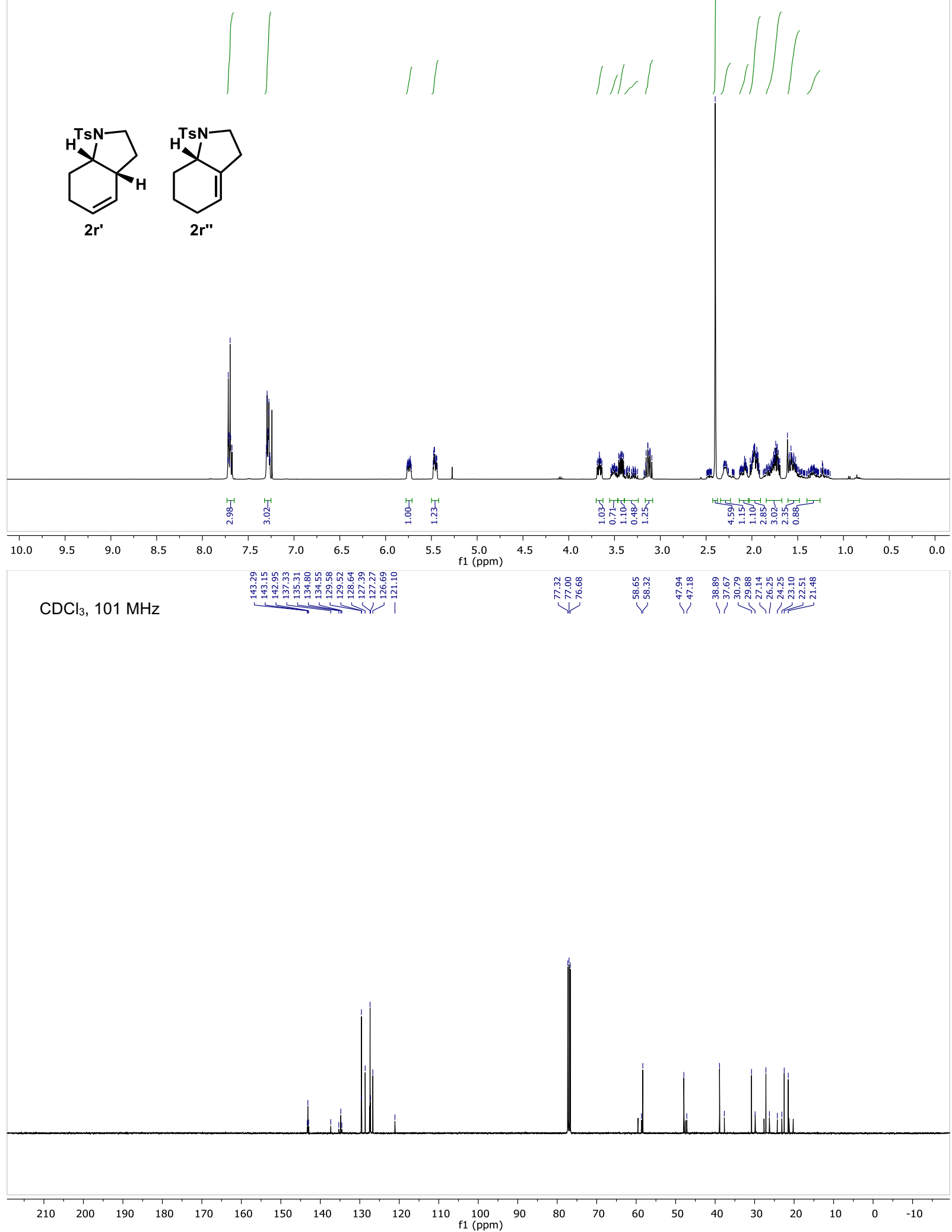


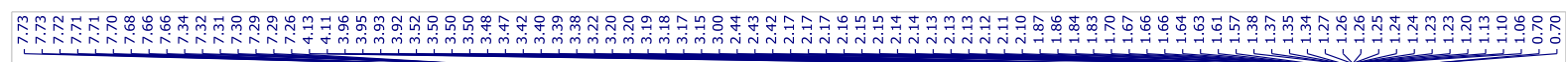
$\mathrm{CDCl}_{3}, 400 \mathrm{MHz}$
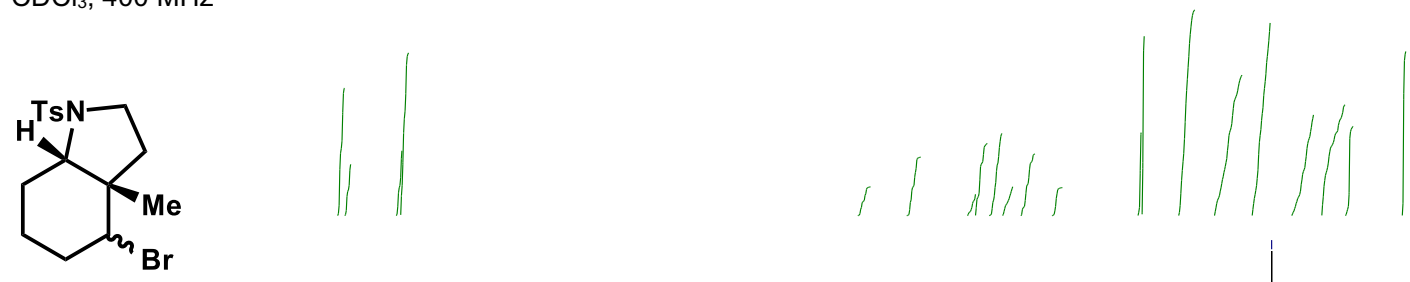

2s

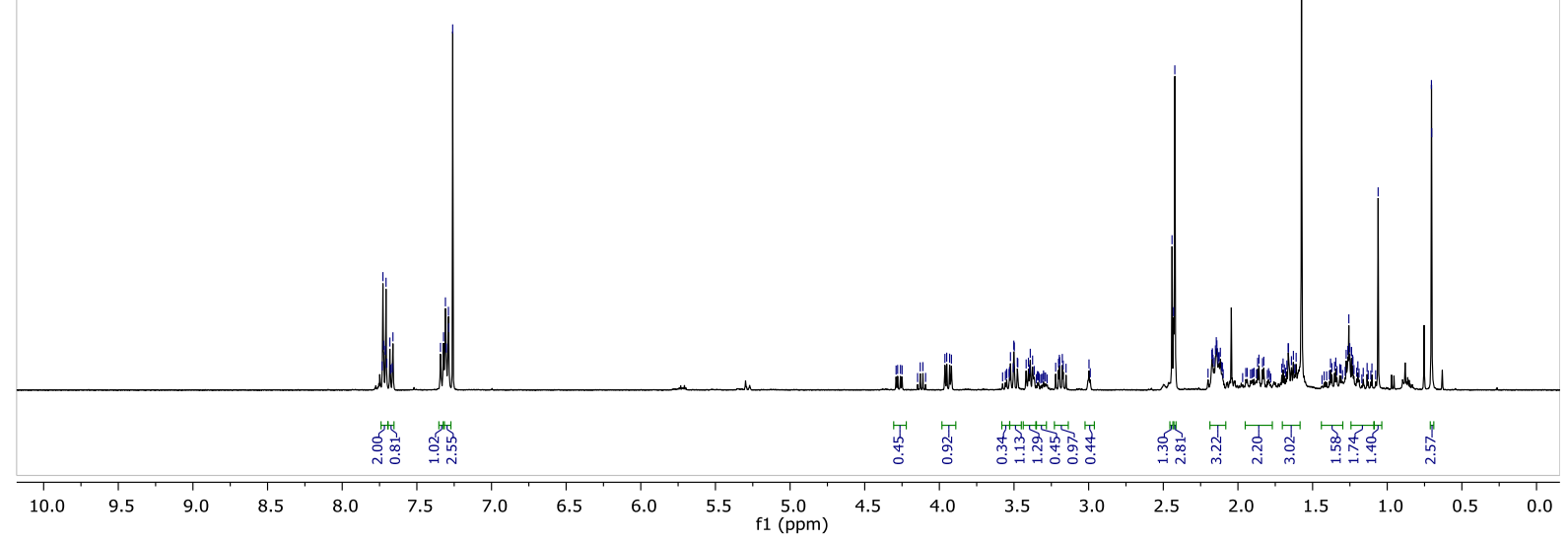

$\mathrm{CDCl}_{3}, 101 \mathrm{MHz}$

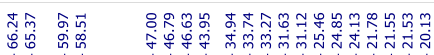

\1|
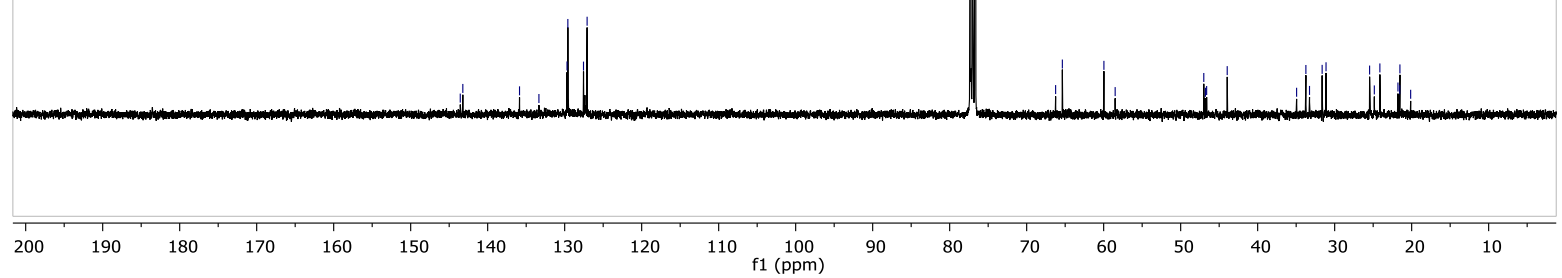


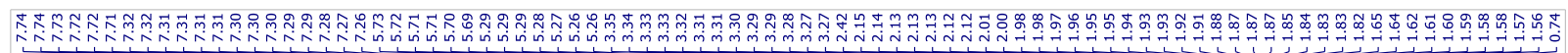

$\mathrm{CDCl}_{3}, 400 \mathrm{MHz}$
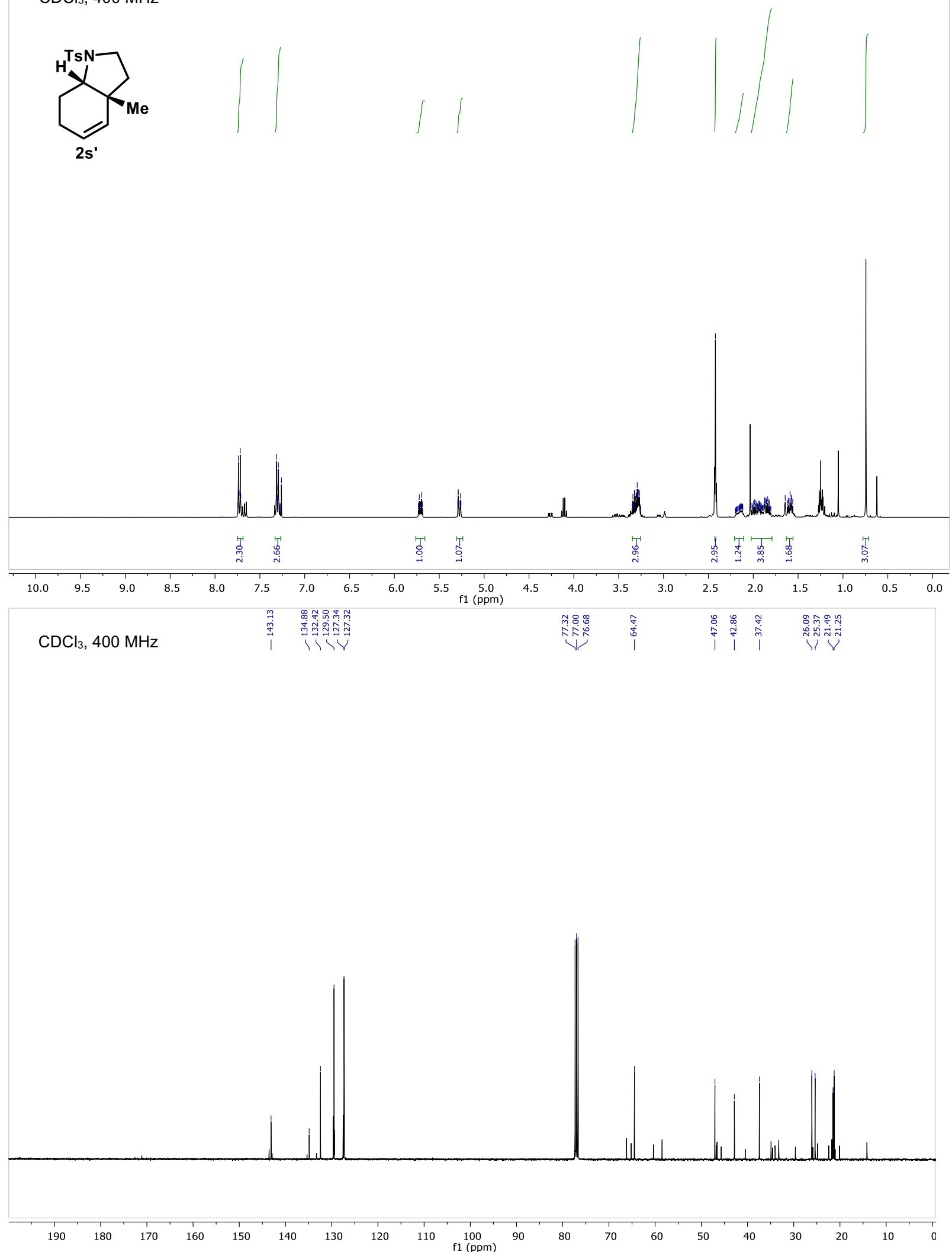


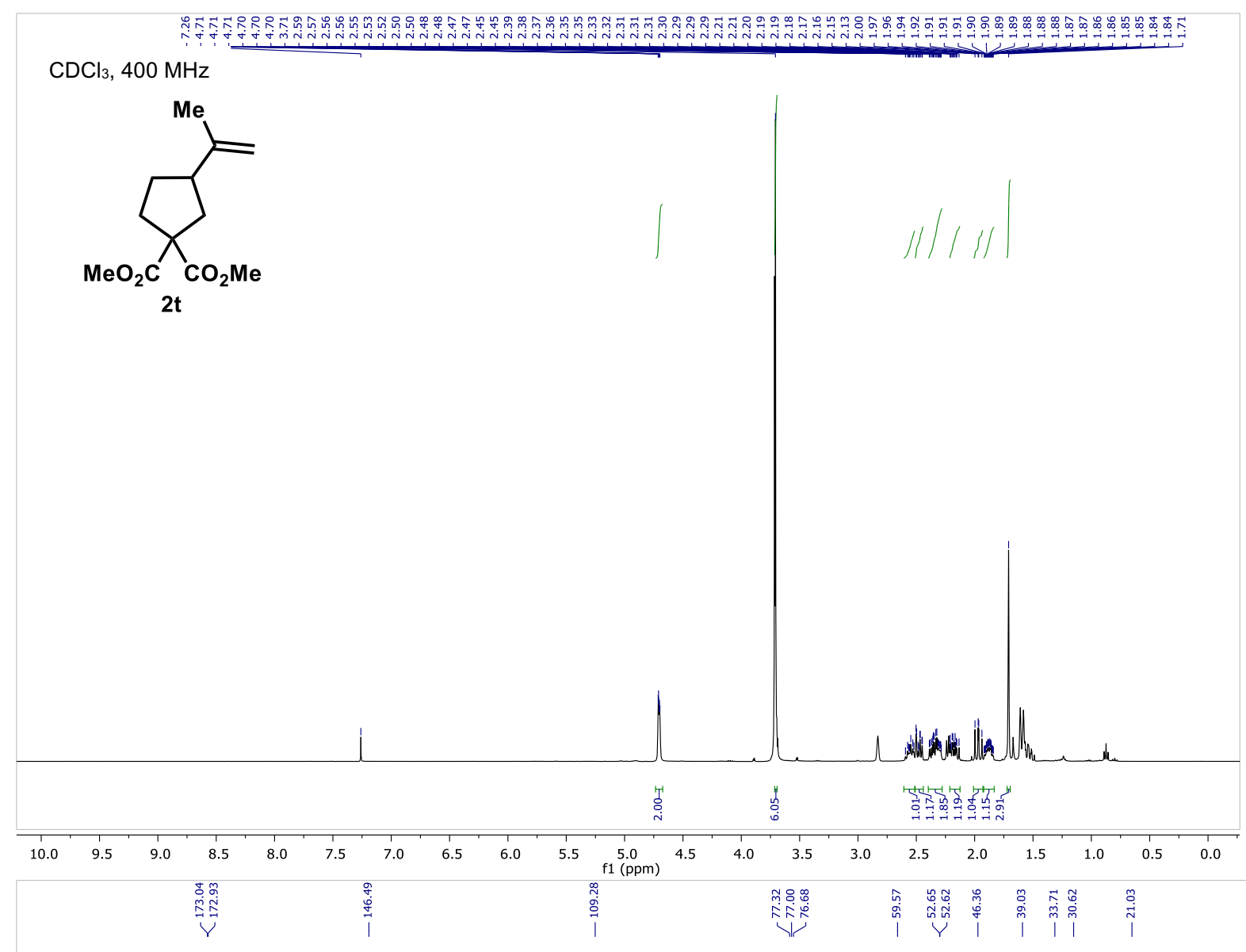

$\mathrm{CDCl}_{3}, 101 \mathrm{MHz}$

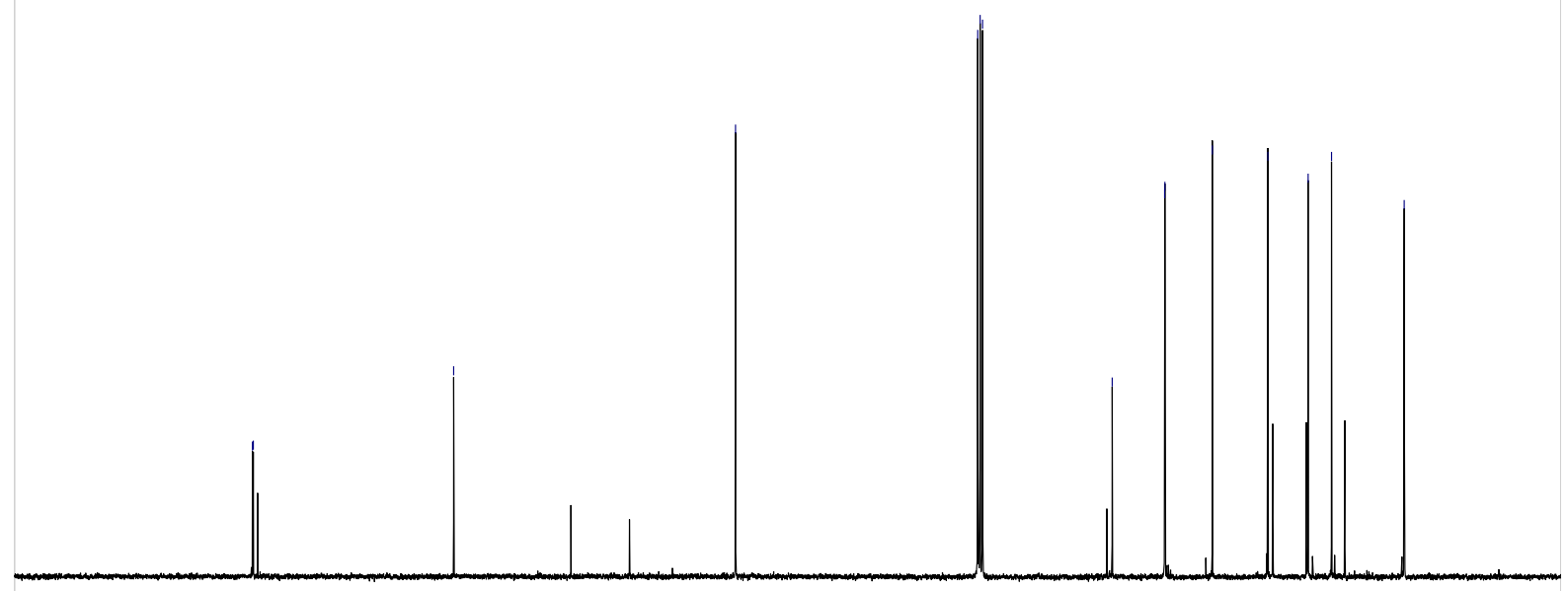

200

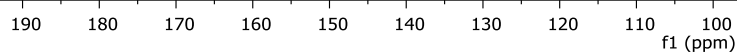




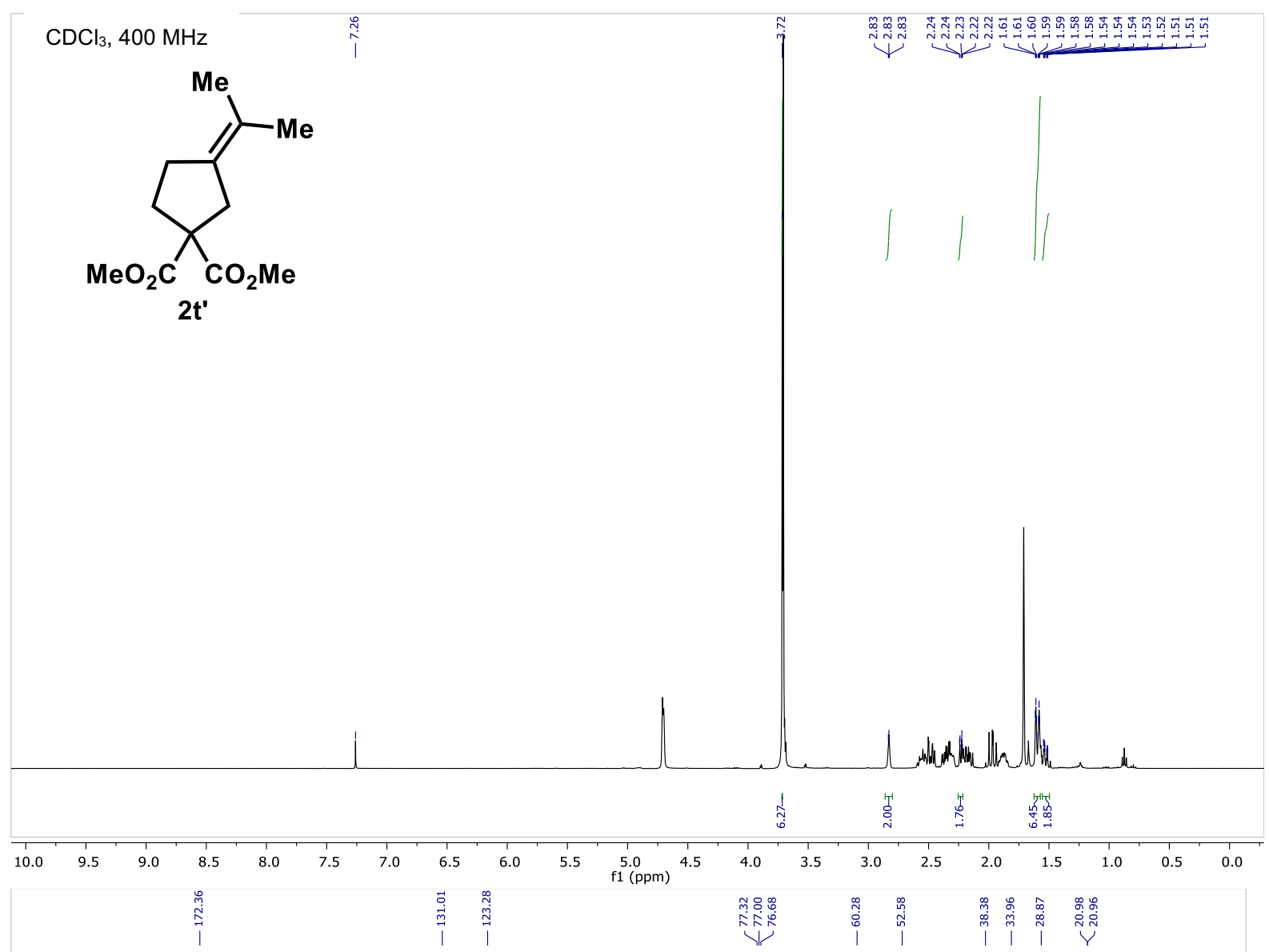

$\mathrm{CDCl}_{3}, 400 \mathrm{MHz}$

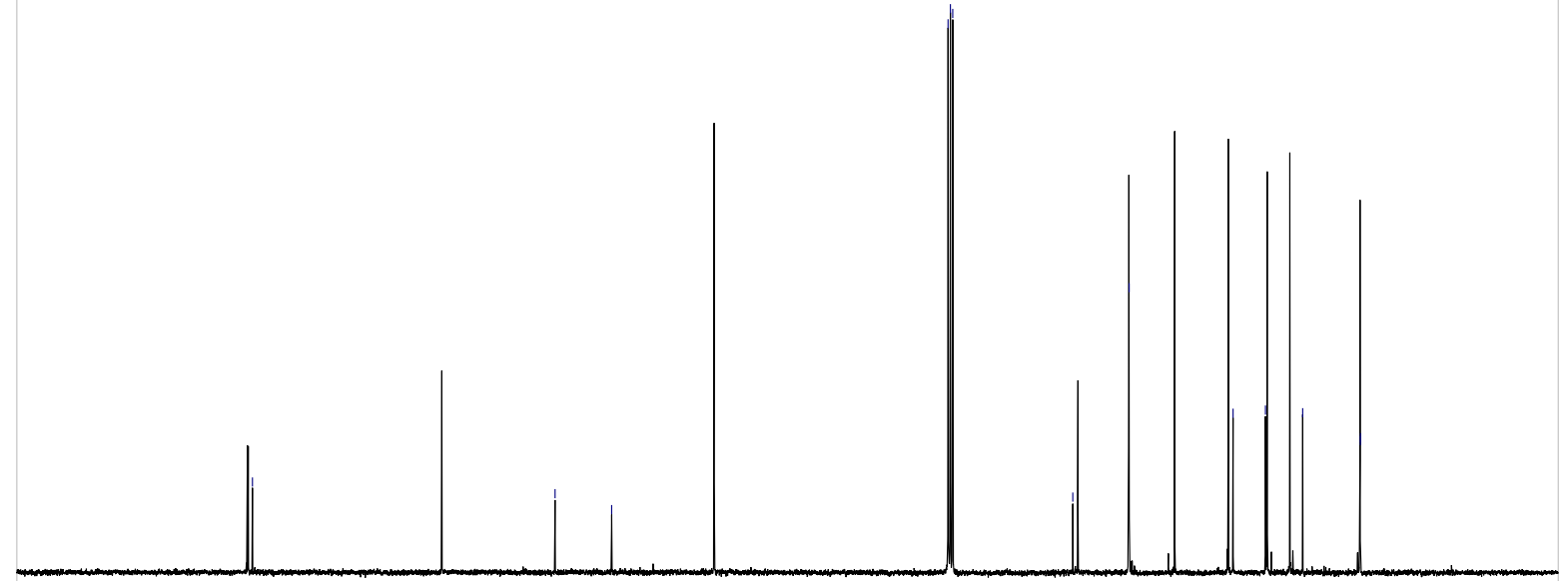

200

$\begin{array}{llll}190 & 180 & 170 & 160\end{array}$

140

$100 \quad 90$ 


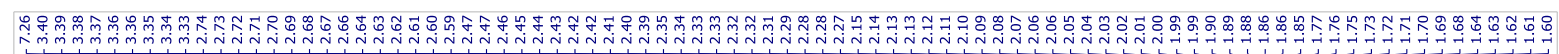

$\mathrm{CDCl}_{3}, 400 \mathrm{MHz}$
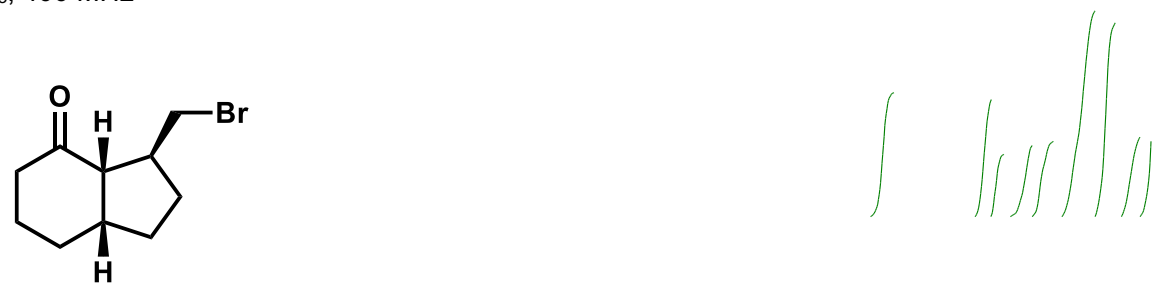

4

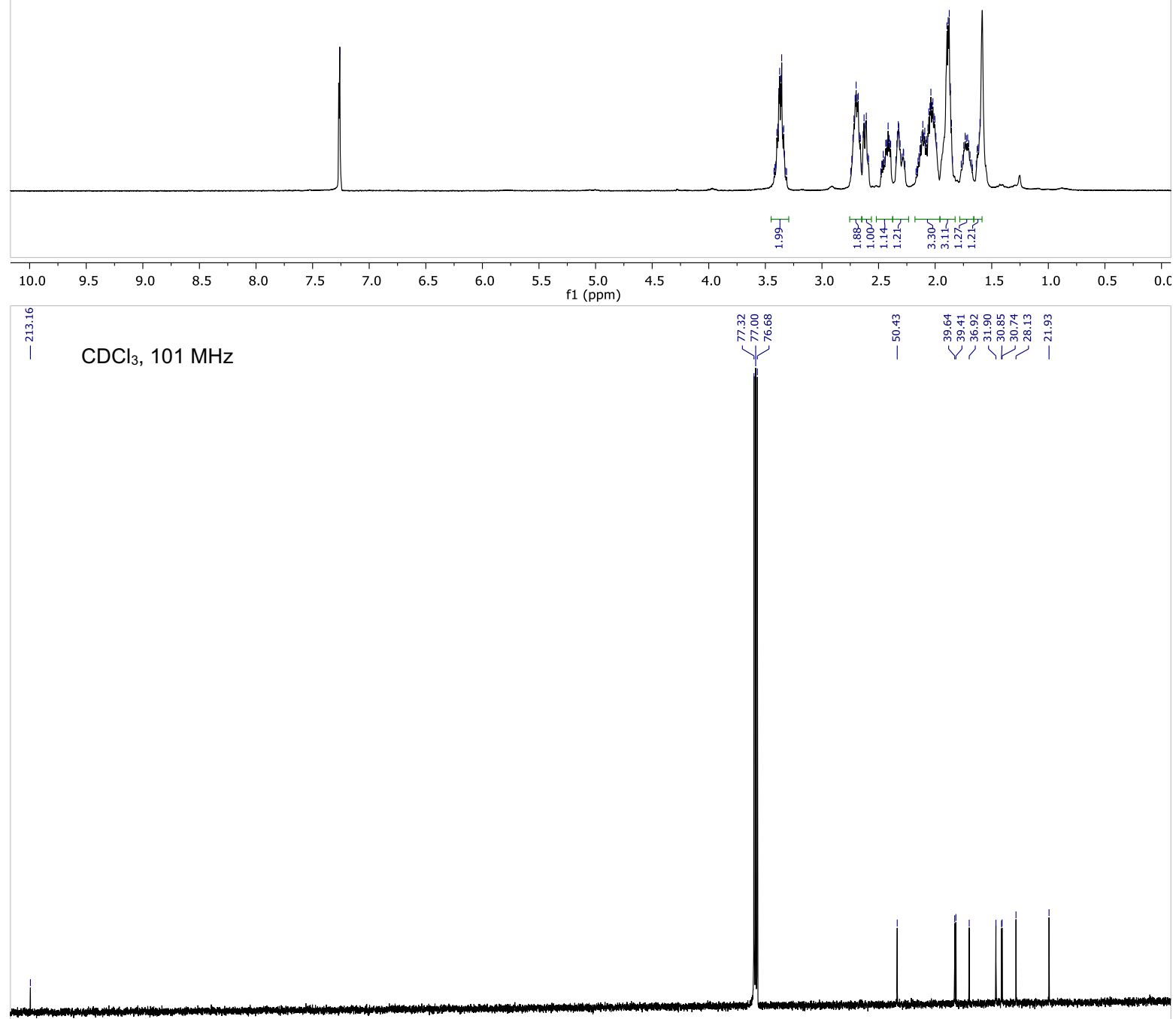

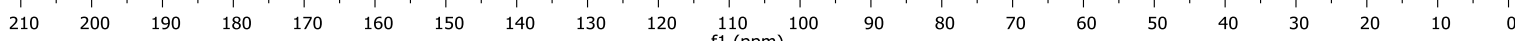




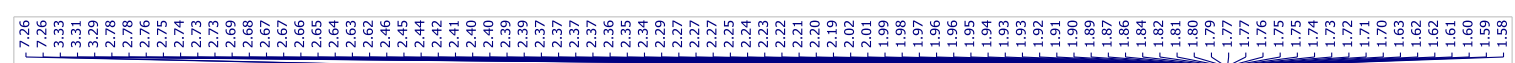
$\mathrm{CDCl}_{3}, 400 \mathrm{MHz}$
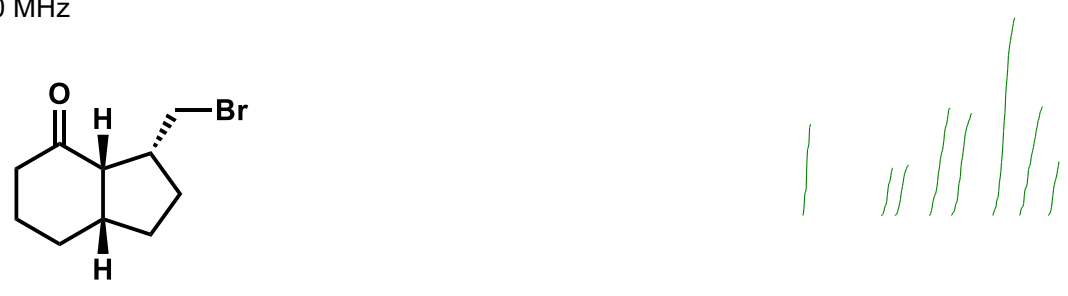

4'
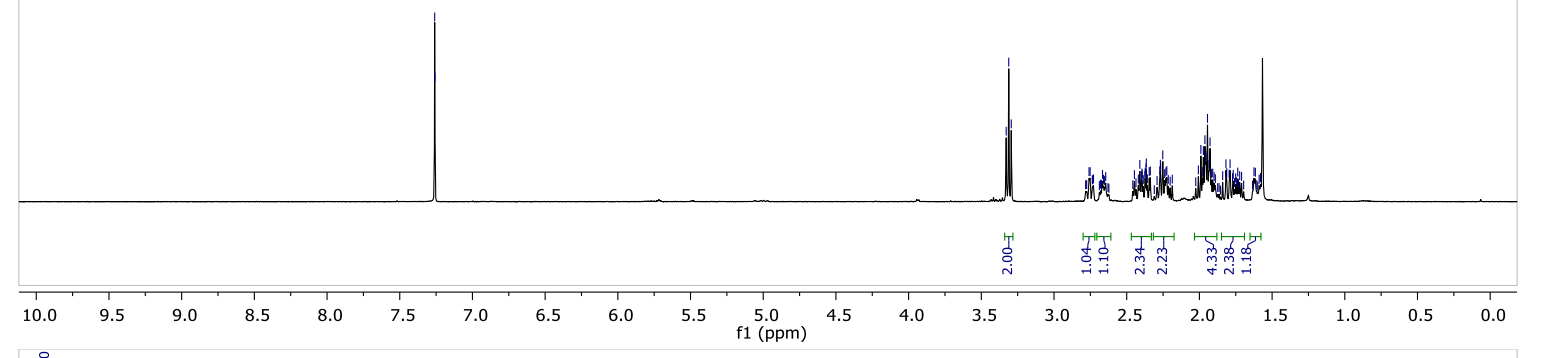

总 $\mathrm{CDCl}_{3}, 101 \mathrm{MHz}$
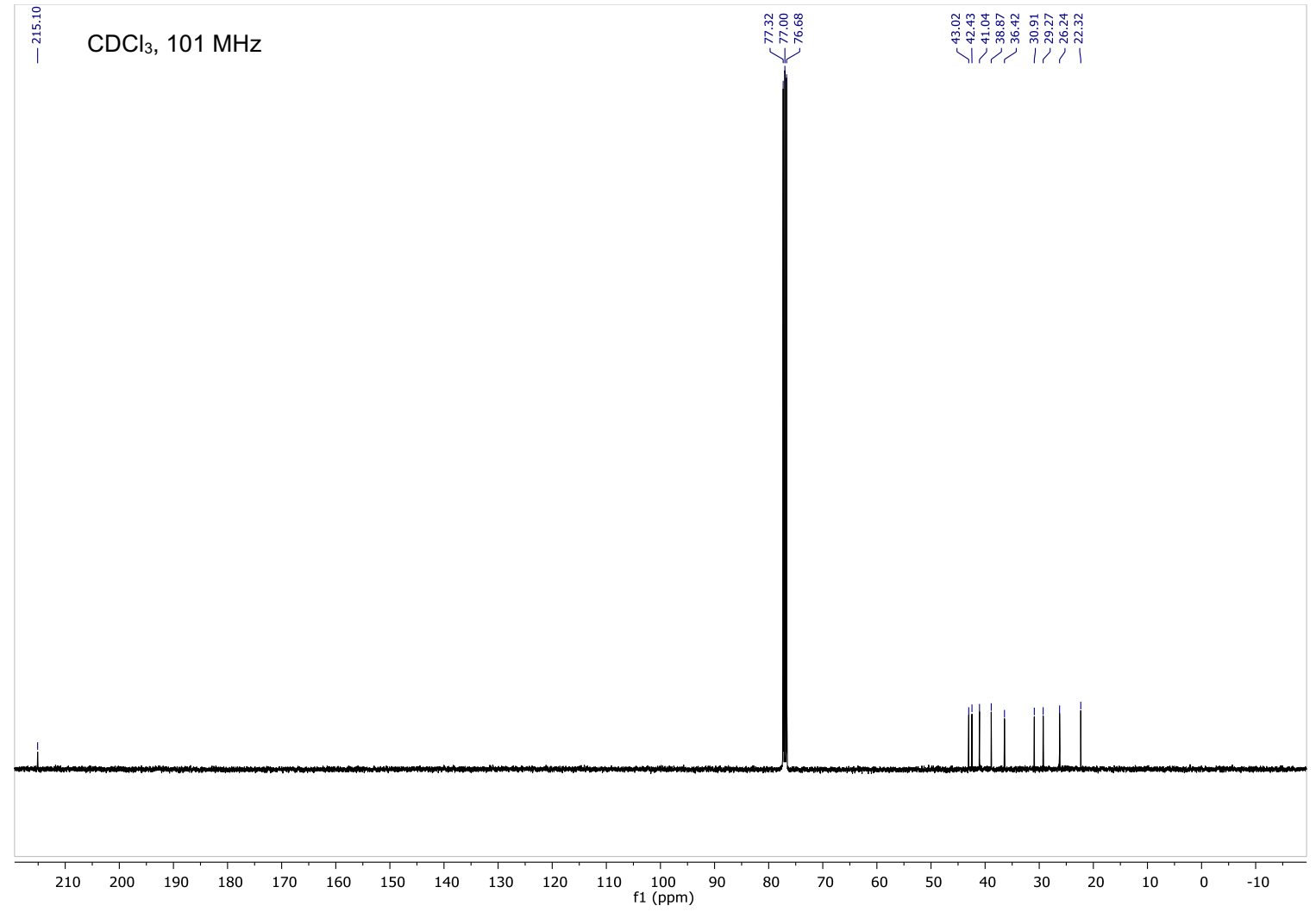